\title{
Invertible Orientation Scores as an Application of Generalized Wavelet Theory ${ }^{\text {II }}$
}

\author{
R. Duits, M. Duits, Markus van Almsick, and B. ter Haar Romeny \\ Department of Biomedical Engineering, Department of Mathematics, Eindhoven University of Technology, \\ P. O. Box 2, $5600 \mathrm{MB}$, Eindhoven The Netherlands \\ e-mail:R.Duits@tue.nl; Maurice.Duits@wis.kuleuven.ac.be;M.v.Almsick@tue.nl; B.M.terhaarRomeny@tue.nl
}

\begin{abstract}
Inspired by the visual system of many mammals, we consider the construction of —and reconstruction from - an orientation score of an image, via a wavelet transform corresponding to the left-regular representation of the Euclidean motion group in $\mathbb{L}_{2}\left(\mathbb{R}^{2}\right)$ and oriented wavelet $\psi \in \mathbb{L}_{2}\left(\mathbb{R}^{2}\right)$. Because this representation is reducible, the general wavelet reconstruction theorem does not apply. By means of reproducing kernel theory, we formulate a new and more general wavelet theory, which is applied to our specific case. As a result we can quantify the well-posedness of the reconstruction given the wavelet $\psi$ and deal with the question of which oriented wavelet $\psi$ is practically desirable in the sense that it both allows a stable reconstruction and a proper detection of local elongated structures. This enables image enhancement by means of left-invariant operators on orientation scores.
\end{abstract}

Keywords: Wavelet theory, Reproducing kernel theory, Euclidean motion group, Euclidean coherent states, Orientation scores, Perceptual organization.

DOI: $10.1134 / \mathrm{S} 1054661807010063$

\section{INTRODUCTION}

In many medical image applications, it is desirable to construct a local orientation-score of a grey-value image. In the case of $2 \mathrm{D}$ images $f: \mathbb{R}^{2} \rightarrow \mathbb{R}$, such an orientation score $U_{f}: \mathbb{R}^{2} \times_{\tau} \mathbb{T} \longrightarrow \mathbb{C}$ depends on three variables $\left(b_{1}, b_{2}, e^{i \theta}\right)$, where $\left(b_{1}, b_{2}\right) \in \mathbb{R}^{2}$ denote position and $e^{i \theta} \in \mathbb{T} \longrightarrow(\cos \theta, \sin \theta) \in S^{1}$ is a local orientation variable. Such an orientation score is usually obtained by means of a convolution with some anisotropic wavelet ${ }^{1} \psi \in \mathbb{L}_{2}\left(\mathbb{R}^{2}\right)$, cf. [21]:

$$
\begin{gathered}
U_{f}\left(\mathbf{b}, e^{i \theta}\right)=\int_{\mathbb{R}^{2}} \overline{\psi\left(R_{\theta}^{-1}\left(\mathbf{x}^{\prime}-\mathbf{b}\right)\right)} f\left(\mathbf{x}^{\prime}\right) d \mathbf{x}^{\prime}, \\
\text { with } R_{\theta}=\left(\begin{array}{cc}
\cos \theta & -\sin \theta \\
\sin \theta & \cos \theta
\end{array}\right) .
\end{gathered}
$$

\footnotetext{
II The text was submitted by the authors in English.

${ }^{1}$ We follow the convention in physics and mathematics to call the kernel corresponding to a wavelet transformation a wavelet. However, in ID-signal analysis, people consider wavelet transformations with respect to representations of the $a x+b$-group (scaling and translation) onto $\mathbb{L}_{2}(\mathbb{R})$. We stress that the interpretation of a wavelet as a localized wave in signal processing does not apply in our case.
}

Received April 5, 2005
This idea is inspired by our own visual system, in which receptive fields exist that are tuned to various locations and orientations. A simple cell receptive field can be parameterized by its position and orientation. Assemblies of oriented receptive fields are grouped together on the surface of the primary visual cortex in a pinwheel-like structure (see Fig. 2).

Perceptual organization on the basis of orientation similarity on images $f$ can be done via their orientation scores $U_{f}$, as long as there exists a linear well-posed invertible transformation $\mathcal{W}_{\psi}$ from the image $f$ to the orientation score $U_{f}$ and vice versa. The domain of $U_{f}$ is the well-known Euclidean motion group $G=\mathbb{R}^{2} \times_{\tau} \mathbb{T}$ and the mapping $f \mapsto U_{f}$ is a wavelet transformation

$$
\begin{gathered}
U_{f}\left(\mathbf{b}, e^{i \theta}\right)=\left(\mathcal{W}_{\psi}[f]\right)(g)=\left(\mathcal{U}_{g} \psi, f\right)_{\mathbb{L}_{2}\left(\mathbb{R}^{2}\right)} \\
=\left(\mathscr{T}_{\mathbf{b}} \mathscr{R}_{\theta} \psi, f\right)_{\mathbb{L}_{2}\left(\mathbb{R}^{2}\right)}, \quad g=\left(\mathbf{b}, e^{i \theta}\right),
\end{gathered}
$$

(a)

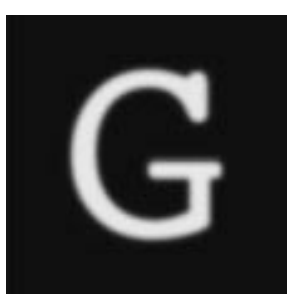

(b)

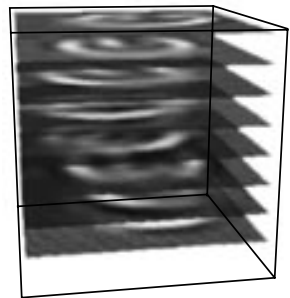

Fig. 1. Example of an orientation score (b) and its corresponding image (a). 
(a)

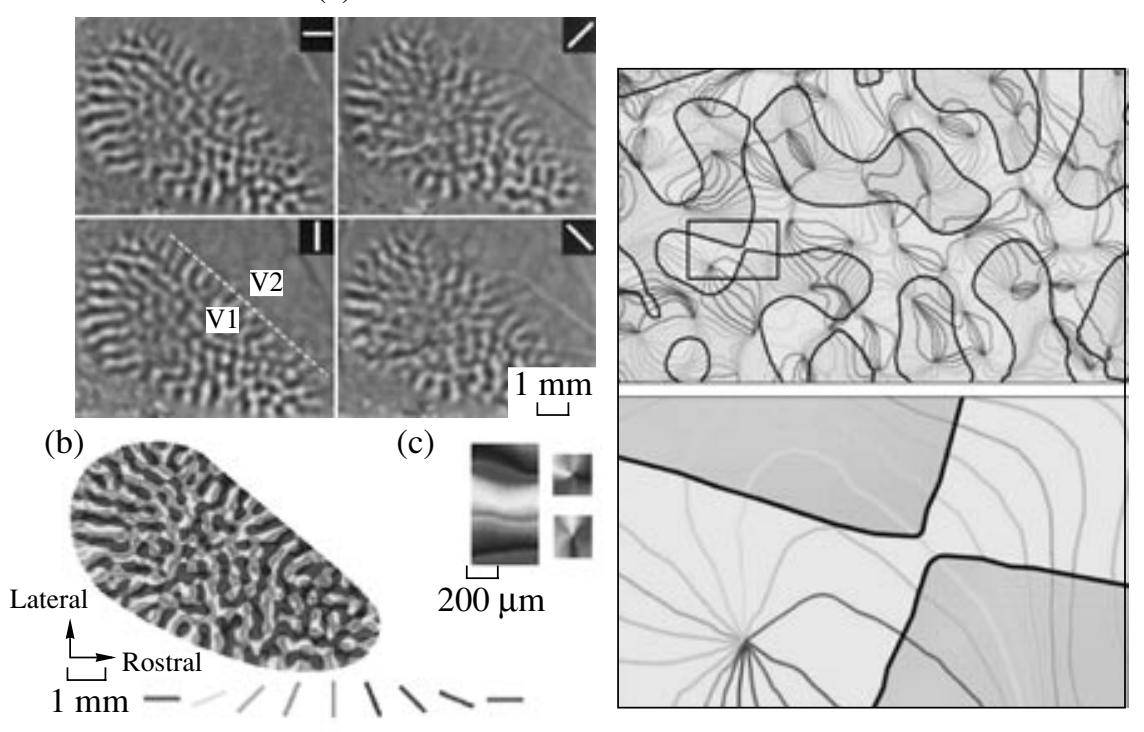

Fig. 2. Left: (a) Parts of visual cortex active under different orientation stimuli. (b) Orientation preference map obtained by vector summation of data obtained for each angle. Orientation preference is color coded according to the key shown below, replicated with permission from [7], Copyright 1997 Society of Neuroscience. Right: enlarged section of the rectangular area in the upper figure. Shaded and unshaded areas denote the left and right eye respectively. Colored lines connect cells with equal orientation sensitivity, replicated with permission from [28].

where $\mathscr{T}_{\mathbf{b}} \mathscr{R}_{\theta} \psi$ is the translated and rotated wavelet and $g \mapsto \mathcal{U}_{g}$ is the left-regular action of $G$ onto $\mathbb{L}_{2}\left(\mathbb{R}^{2}\right)$. The definitions and conventions are explained in Section 2 (see (2.4), (2.6), and (2.7)).

In Section 3 we give a brief overview of the standard wavelet theory. Although this theory guarantees a perfectly well-posed reconstruction of $f$ from its wavelet transform $\mathcal{W}_{\psi}[f]$, in the sense that the quadratic norm is preserved, it does not apply to our case.

Therefore, in Section 4 we give a brief overview of our recently developed more general approach to wavelet theory (by means of reproducing kernel theory), leading to a more general wavelet reconstruction theorem, which is necessary for the application of our orientation score. This theory (which is put in a much more general framework) answers the questions that inevitably arise from the inspiring work of Kalitzin et al. [21] on invertible orientation scores. For more detailed in-depth mathematical treatment of this recently developed theory, we refer to the earlier work [10].

In Section 5, we consider the practical consequences of this reconstruction theorem and quantify the wellposedness of reconstruction by a condition number that explicitly depends on the wavelet $\psi$. We follow two different approaches to parameterize classes of wavelets which allow a stable reconstruction and give some explicit examples. These examples show that it is possible to derive wavelets that both allow a well-posed reconstruction from a single scale orientation score (which is not possible in the usual wavelet approaches!) and which are at the same time good line detectors. We compare these wavelets with the usual line detectors used in image analysis, which do not allow a stable reconstruction. In Section 7 we generalize our results to orientation scores of $3 D$-images and even obtain wellposed invertible orientation scores of $3 D$-images.

In Section 8 we give explicit practical examples of perceptual organization by means of left-invariant operations on orientation scores. In these examples we first construct an orientation score $U_{f}=\mathcal{W}_{\psi}[f]$, then apply an operation $\Phi$ on the orientation score $\Phi\left[U_{f}\right]$ after which we reconstruct to obtain an enhanced image $\Upsilon_{\psi}[f]=\left[W_{\psi}^{*} \circ \Phi \circ W_{\psi}\right](f)$. We show that the concatenation

$$
\Upsilon_{\psi}=W_{\psi}^{*} \circ \Phi \circ W_{\psi}
$$

is Euclidean invariant if and only if $\Phi$ is left-invariant.

\section{PRELIMINARIES AND NOTATION}

- Images/signals are assumed to be within $\mathrm{L}_{2}\left(\mathbb{R}^{d}\right)$. For signals, $d=1$. For images, $d=2$, unless explicitly stated otherwise.

- The Fourier transform $\mathscr{F}: \mathbb{L}_{2}\left(\mathbb{R}^{d}\right) \longrightarrow \mathbb{L}_{2}\left(\mathbb{R}^{d}\right)$ is almost everywhere defined by

$$
[\mathscr{F}(f)](\boldsymbol{\omega})=\hat{f}(\boldsymbol{\omega})=\frac{1}{(2 \pi)^{d / 2}} \int_{\mathbb{R}^{d}} f(\mathbf{x}) e^{-i \boldsymbol{\omega} \cdot \mathbf{x}} d \mathbf{x} .
$$


Notice that $\|\mathscr{F}[f]\|^{2}=\|f\|^{2}$ and $\mathscr{F}[f * g]=$ $(2 \pi)^{d / 2 \mathscr{F}}[f] \mathscr{F}[g]$ for all $f, g \in \mathbb{L}_{2}\left(\mathbb{R}^{d}\right)$.

- We use the following notation for Euclidean/polar coordinates in the spatial and Fourier domains, respectively: $\mathbf{x}=(x, y)=(r \cos \phi, r \sin \phi), \boldsymbol{\omega}=\left(\omega_{x}, \omega_{y}\right)=$ $(\rho \cos \varphi, \rho \sin \varphi)$, with $\phi, \varphi \in[0,2 \pi), r, \rho>0$. The corresponding complex variables will be denoted by $z=$ $x+i y=r e^{i \phi}$ and $w=\omega_{x}+i \omega_{y}=\rho e^{i \varphi}$.

- We will use short notation for the following groups:

—Aut $\left(\mathbb{R}^{d}\right)=\left\{A: \mathbb{R}^{d} \longrightarrow \mathbb{R}^{d} \mid A\right.$ linear and $A^{-1}$ exists $\}$ $a>0\}$.

-dilation group $D(d)=\left\{A \in \operatorname{Aut}\left(\mathbb{R}^{d}\right) \mid A=a I\right.$,

—orthogonal group $O(d)=\left\{X \in \operatorname{Aut}\left(\mathbb{R}^{d}\right) \mid X^{T}=X^{-1}\right\}$

一rotation group $S O(d)=\{R \in O(d) \mid \operatorname{det}(R)=1\}$.

—circle group $\mathbb{T}=\{z \in \mathbb{C} \| z \mid=1\}, z=e^{i \theta}, \theta=\arg z$ with group homomorphism $\tau: \mathbb{T} \longrightarrow S O(2) \subset \operatorname{Aut}\left(\mathbb{R}^{2}\right)$ :

$$
\tau(z)=R_{\theta}=\left(\begin{array}{cc}
\cos \theta & -\sin \theta \\
\sin \theta & \cos \theta
\end{array}\right) .
$$

- Let $T$ and $S$ be locally compact groups and let $\tau: T \longrightarrow \operatorname{Aut}(S)$ be a group homomorphism. The semidirect product $S \times{ }_{\tau} T$ is defined to be the group (which is again locally compact) with underlying set $\{(s, t) \mid s \in$ $S, t \in T\}$ and group operation

$$
(s, t)\left(s^{\prime}, t^{\prime}\right)=\left(s \tau(t) s^{\prime}, t t^{\prime}\right) .
$$

In this article, we mainly consider the group $\mathbb{R}^{2} \times_{\tau} \mathbb{T}$, where $\tau$ is given by (2.4). The group product (2.5) is now given by

$$
\begin{aligned}
g g^{\prime} & =\left(\mathbf{b}, e^{i \theta}\right)\left(\mathbf{b}^{\prime}, e^{i \theta}\right)=\left(\mathbf{b}+R_{\theta} \mathbf{b}^{\prime}, e^{i\left(\theta+\theta^{\prime}\right)}\right), \\
g & =\left(\mathbf{b}, e^{i \theta}\right), \quad g^{\prime}=\left(\mathbf{b}^{\prime}, e^{i \theta^{\prime}}\right) \in \mathbb{R}^{2} \times_{\tau} \mathbb{T} .
\end{aligned}
$$

This non-commutative group is the Euclidean motion group.

- With $\mathscr{B}(H)$, we denote the space of bounded operators on $H$. The range of a linear operator $A$ will be denoted by $\mathscr{R}(A)$ and its nilspace will be denoted by $\mathcal{N}(A)$.

- A representation $\mathscr{R}$ of a group $G$ onto a Hilbert space $H$ is a homomorphism $\mathscr{R}$ between $G$ and $\mathscr{B}(H)$, the space of bounded linear operators on $H$. It satisfies $\mathscr{R}_{g h}=\mathscr{R}_{g} \mathscr{R}_{h}$ for all $g \in G, h \in G$ and $\mathscr{R}_{e}=I$. A representation $\mathscr{R}$ is irreducible if the only closed invariant subspaces of $H$ are $H$ and $\{0\}$; otherwise it is reducible. We mainly consider unitary representations (i.e., $\left\|u_{g}\right\|_{H}=\|\psi\|_{H}$ for all $g \in G$ and $\psi \in H$ ), which will be denoted by $\mathcal{U}$ rather than $\mathscr{R}$. Within the class of unitary representations, we are mainly interested in the representations of $\mathbb{R}^{d} \times_{\tau} T$ onto $\mathbb{L}_{2}\left(\mathbb{R}^{d}\right)$ which are given by

$$
\left(U_{g} \psi\right)(\mathbf{x})=\frac{1}{\sqrt{\operatorname{det}(\tau(t))}} \psi\left(\left(\tau^{-1}(t)\right)(\mathbf{x}-\mathbf{b})\right) .
$$

We will call these representations left-regular actions of $G=\mathbb{R}^{d} \times_{\tau} T$ in $\mathbb{L}_{2}\left(\mathbb{R}^{d}\right)$.

- Let $\mathbf{b} \in \mathbb{R}^{d}, a>0$ and $g \in G \longleftrightarrow \tau(G) \subset \operatorname{Aut}\left(\mathbb{R}^{d}\right)$. Then the unitary operators $f \mapsto \check{f}, \tau_{\mathbf{b}}, \mathscr{D}_{a}$ and $\mathscr{R}_{g}, \mathbf{b} \in$ $\mathbb{R}^{d}, a>0$ on $\mathbb{L}_{2}\left(\mathbb{R}^{d}\right)$ are defined by

$$
\begin{gathered}
f(\mathbf{x})=f(-\mathbf{x}) \quad \mathscr{T}_{\mathbf{b}} \psi(\mathbf{x})=\psi(\mathbf{x}-\mathbf{b}) \\
\left(\mathscr{D}_{a} \psi\right)(\mathbf{x})=\frac{1}{a^{\frac{d}{2}}} \psi\left(\frac{\mathbf{x}}{a}\right) \\
\mathscr{R}_{g} \psi(\mathbf{x})=\frac{1}{\sqrt{\operatorname{det} \tau(g)}} \psi\left((\tau(g))^{-1} \mathbf{x}\right),
\end{gathered}
$$

which are left regular actions of $O(1), \mathbb{R}^{d}, D(d), G$ in $\mathbb{L}_{2}\left(\mathbb{R}^{d}\right)$.

- A functional Hilbert space ${ }^{2}$ is a Hilbert space consisting of complex valued functions on an index set \ on which the point evaluation $\delta_{a}$ is a continuous/bounded linear functional for all $\mathbf{a} \in \mathbb{\square}$. Consequently, it has a Riesz representant $K_{\mathrm{a}} \in H$

$$
f(\mathbf{a})=\left\langle\delta_{\mathbf{a}}, f\right\rangle=\left(K_{\mathbf{a}}, f\right)_{H} .
$$

The function $K: \square \times \square \longrightarrow \mathbb{C}$ given by $K(\mathbf{a}, \mathbf{b})=\left(K_{\mathrm{a}}\right.$, $\left.K_{\mathbf{b}}\right)_{H}=K_{\mathbf{b}}(\mathbf{a})$ is called reproducing kernel. Notice that the spaces $\mathbb{L}_{2}\left(\mathbb{R}^{d}\right)$ are not functional Hilbert spaces.

- The $d$-dimensional Gaussian kernel $G_{s}$ at scale $\mathrm{s}$ is given by

$$
G_{s}(\mathbf{x})=\frac{1}{(4 \pi s)^{d / 2}} e^{-\frac{\|\mathbf{x}\|^{2}}{4 s}} .
$$

Occasionally, we will parameterize the Gaussian kernel by its standard deviation $\sigma$ and write $\mathscr{G}_{\sigma}=G_{s}$, where we notice that $s=\frac{\sigma^{2}}{2}$.

- For a given wavelet $\psi \in \mathbb{L}_{1}\left(\mathbb{R}^{d}\right) \cap \mathbb{L}_{2}\left(\mathbb{R}^{d}\right)$, we define $\tilde{\psi}$ and $M_{\psi}$ almost everywhere by

$$
\begin{gathered}
\tilde{\psi}(\mathbf{x})=\int_{S O(d)}\left(\overline{\mathscr{R}_{t} \check{\psi}} * \mathscr{R}_{t} \psi\right)(\mathbf{x}) d \mu_{T}(t) \\
M_{\psi}(\boldsymbol{\omega})=(2 \pi)^{d / 2} \int_{S O(d)}\left|\mathscr{F}_{(}\left(\mathscr{R}_{t} \psi\right)(\boldsymbol{\omega})\right|^{2} d \mu_{T}(t),
\end{gathered}
$$

\footnotetext{
${ }^{2}$ Also known as the reproducing kernel Hilbert space.
} 
where $d \mu_{T}(t)$ is the normalized left-invariant Haar-measure of $S O(d)$. It directly follows from the compactness of $S O(d)$ and Fubini that $M_{\psi} \in \mathbb{L}_{1}\left(\mathbb{R}^{d}\right)$ and

$$
\begin{aligned}
\left\|M_{\psi}\right\|_{\mathbb{R}_{1}\left(\mathbb{R}^{d}\right)} & =(2 \pi)^{d / 2}\|\mathscr{F}[\psi]\|_{\mathbb{L}_{1}\left(\mathbb{R}^{d}\right)}^{2} \int_{S O(d)} d \mu_{T}(t) \\
& =(2 \pi)^{d / 2}\|\psi\|_{\mathbb{L}_{1}\left(\mathbb{R}^{d}\right)}^{2}<\infty .
\end{aligned}
$$

Consequently, the kernel $\tilde{\psi}$, which is the inverse Fourier transform of $M_{\psi}$, is a continuous function vanishing at infinity. Moreover, $M_{\psi}$ and $\tilde{\psi}$ are isotropic since $\mathscr{R}_{t} M_{\psi}=M_{\psi}$ for all $t \in S O(d)$ and $\mathscr{F}_{\mathscr{R}} \mathscr{R}_{t}=\mathscr{R}_{t} \mathscr{F}$. Conversely, by compactness of $S O(d)$ and since a convolution of two $\mathbb{L}_{1}\left(\mathbb{R}^{d}\right)$ elements is again within $\mathbb{L}_{1}\left(\mathbb{R}^{d}\right)$, we obtain that $\tilde{\psi} \in \mathbb{L}_{1}\left(\mathbb{R}^{d}\right)$, so $M_{\psi}$ is also a continuous function vanishing at infinity.

\section{WAVELET THEORY}

In this Section, we will give a condensed treatment of continuous wavelet theory. For the sake of illustration, we will first consider the well-known signal case, i.e., $d=1$ with $G=\mathbb{R} \times D(1)$ known as the $a x+b$-group. Then we give the group theoretic generalization first formulated by Grossmann et al. [19]. This theorem and other generalizations of this theorem, such as [29], [17] are not applicable to the reducible left regular action of the Euclidean motion group onto $\mathbb{L}_{2}\left(\mathbb{R}^{d}\right)$ given by (2.6), which is needed for our orientation score application in image analysis. Therefore, we give a true generalization to the wavelet theorem, where irreducibility is neither a requirement nor replaced by another requirement.

\subsection{Continuous Wavelet Theory on 1D-Signals}

The continuous wavelet transform $\mathcal{W}_{\psi}[f]: \mathbb{R}^{+} \times$ $\mathbb{R} \longrightarrow \mathbb{C}$ of a signal $f \in \mathbb{L}_{2}(\mathbb{R})$ with respect to wavelet $\psi \in \mathbb{L}_{2}(\mathbb{R})$ is defined by $\mathcal{W}_{\psi}[f](a, b)=\left(\mathscr{T}_{b} \mathscr{D}_{a}, f\right)_{\mathbb{L}_{2}(\mathbb{R})}$. An alternative expression of the continuous wavelet transform is given by $\mathscr{W}_{\psi}[f](a, b)=\left(f * \overline{\mathscr{D}_{a} \check{\psi}}\right)(b)$. We say $\psi \in \mathbb{Z}_{2}(\mathbb{R})$ is an admissible wavelet if it satisfies the condition $^{3}$

$$
0<C_{\psi}=(2 \pi) \int_{\mathbb{R}^{+}} \frac{|\mathscr{F}[\psi](a \omega)|^{2}}{a} d a<\infty
$$

for almost all $\omega \in \mathbb{R}$.

\footnotetext{
${ }^{3}$ If $\psi(-\mathbf{x})=e^{i \alpha} \psi(\mathbf{x})$, this expression docs not depend on $\omega$. Then $C_{\psi}<\infty \Leftrightarrow \mathscr{F}[\psi](0)=\int_{\mathbb{R}} \psi(x) d x=0$.
}

Notice that $\mathscr{T}_{b} \mathscr{D}_{a} \circ \mathscr{T}_{b^{\prime}} D_{a^{\prime}}=\mathscr{T}_{a b^{\prime}+b} \mathscr{D}_{a a^{\prime}}$ and that $\left(\mathscr{T}_{b} \mathscr{D}_{a}\right)^{-1}=\left(\mathscr{T}_{-b / a} \mathscr{D}_{1 / a}\right)$ and therefore $\left\{\mathscr{T}_{b} \mathscr{D}_{a}\right\}$ forms a 2-parameter Lie group $G^{+}$, known as the $a x+b$-group, isomorphic to the matrix group

$$
\left\{\left(\begin{array}{ll}
a & b \\
0 & 1
\end{array}\right) \mid a>0, b \in \mathbb{R}\right\} \text {. }
$$

Let $U$ be the representation of $G^{+}$given by

$$
\left[\mathcal{U}_{(a, b)} f\right](x)=\frac{1}{\sqrt{a}} f\left(\frac{x-b}{a}\right) ;
$$

then $\mathcal{U}$ is unitary, i.e., $\left(\boldsymbol{U}_{(a, b)} f, \boldsymbol{U}_{(a, b)} g\right)=(f, g)$ for all $a>0$ and $b \in \mathbb{R}$, and $\mathcal{W}_{\psi}[f](a, b)=\left(\mathcal{U}_{(a, b)} \psi, f\right)_{\mathbb{L}_{2}\left(\mathbb{R}^{d}\right)}$. By straightforward computation, it follows that the wavelet constant $C_{\psi}$ can be rewritten:

$$
\begin{gathered}
C_{\psi}=\frac{1}{(\psi, \psi)} \int_{G^{+}}\left|\left(\mho_{g} \psi, \psi\right)\right|^{2} d g \\
=\frac{1}{(\psi, \psi)} \int_{G^{+}}\left|\left(U_{(a, b)} \psi, \psi\right)\right|^{2} \frac{d a}{a^{2}} d b,
\end{gathered}
$$

where the measure $d \mu_{G^{+}}(g)=\frac{d a}{a^{2}} d b$ is the left-invariant Haar measure of $G^{+}$.

Theorem 3.1 (Wavelet Reconstruction Theorem for Signals)

Let $\psi \in \mathbb{L}_{2}(\mathbb{R})$ be an admissible wavelet, with wavelet constant ${ }^{4} C_{\psi}=(2 \pi) \int \frac{|\hat{\psi}(a \omega)|^{2}}{a} d a<\infty$ for almost all $\omega \in \mathbb{R}$. Then the mapping $\Phi$ which maps $f$ onto $\frac{1}{\sqrt{C_{\psi}}} \mathcal{W}_{\psi}[f]$ is a unitary mapping from $\mathbb{L}_{2}(\mathbb{R})$ into $\mathbb{L}_{2}\left(\mathbb{R} \times \mathbb{R}^{+}\right)$, i.e., $\left\|W_{\psi}[f]\right\|_{\mathbb{L}_{2}\left(\mathbb{R} \times \mathbb{R}^{+} ; d b \frac{d a}{a^{2}}\right)}^{2}=C_{\psi}\|f\|_{\mathbb{L}_{2}(\mathbb{R})}^{2}$ for all $f \in \mathbb{L}_{2}(\mathbb{R})$ and thereby

$$
\begin{gathered}
f(x)=\frac{1}{\sqrt{C_{\psi}}} \mathscr{W}_{\psi}^{*}\left[\mathcal{W}_{\psi}[f]\right](x) \\
=\frac{1}{\sqrt{C_{\psi}}} \int_{\mathbb{R}^{+}}\left(\left(f * \overline{\mathscr{D}_{a} \check{\psi}}\right) * \mathscr{D}_{a} \psi\right)(x) \frac{d a}{a^{2}}, \\
\text { for almost every } x \in \mathbb{R} .
\end{gathered}
$$

\footnotetext{
${ }^{4}$ This is a constant if $\psi(-\mathbf{x})=e^{i \alpha} \psi(\mathbf{x})$, for some $\alpha \in[0,2 \pi)$ and almost every $x \in \mathbb{R}$.
} 
Proof. This follows from the general result in the next Subsection. Theorem 3.2: Take $H=\mathbb{L}_{2}(\mathbb{R}), G=$ $G^{+}=\mathbb{R} \times D(1) \times O(1)$ and the unitary representation $\mathscr{V}$ of $G$ on $\mathbb{L}_{2}(\mathbb{R})$ by

$$
\begin{gathered}
{\left[\mathscr{V}_{(a, b, c)} f\right](x)=\frac{1}{\sqrt{a}} f\left(\frac{c^{-1}(x-b)}{a}\right),} \\
c \in\{-1,1\}, \quad a>0, \quad b \in \mathbb{R}, \quad f \in \mathbb{L}_{2}(\mathbb{R}) .
\end{gathered}
$$

Notice to this end that in contrast to the representation $\mathcal{U}$ given by (3.12), representation $\mathscr{V}$ is irreducible. Suppose there exists a subspace $S$ of $H$ which is invariant under $\mathscr{V}$. In particular, since $\mathscr{V}_{(a, b, 1)}=\mathcal{U}_{(a, b)}$ it is also invariant under $\mathcal{U}$. Now we notice that $\mathbb{L}_{2}\left(\mathbb{R}^{d}\right)=$ $H_{+} \oplus H_{-}$is an orthogonal decomposition into $U$-irreducible subspaces, [2]. So either $S=\{0\}$ or $S=H$ or $S=H_{+}=\left\{f \in \mathbb{L}_{2}\left(\mathbb{R}^{d}\right) \mid \hat{f}(\omega)=0\right.$ for $\left.\omega>0\right\}$ or $S=H_{-}=$ $\left\{f \in \mathbb{L}_{2}\left(\mathbb{R}^{d}\right) \mid \hat{f}(\omega)=0\right.$ for $\left.\omega<0\right\}$. But $\mathscr{V}_{a, b,-1} H_{+}=H_{-}$ and $\mathscr{V}_{a, b,-1} H_{-}=H_{+}$and therefore $S=\{0\}$ or $S=H$. As a result, by Theorem 3.2 we have

$$
\begin{gathered}
\iint_{\mathbb{R}_{\mathbb{R}^{+}}} \frac{1}{2} \sum_{c \in\{-1,1\}}\left|\left(\mathscr{V}_{(a, b, c)} \psi, f\right)\right|^{2} d b \frac{d a}{a^{2}} \\
=\frac{\|f\|^{2}}{(\psi, \psi)} \iint_{\mathbb{R}_{\mathbb{R}^{+}}} \frac{1}{2} \sum_{c \in\{-1,1\}}\left|\left(\mathscr{V}_{(a, b, c)} \psi, \psi\right)\right|^{2} d b \frac{d a}{a^{2}},
\end{gathered}
$$

and since it is assumed to be admissible with wavelet constant $C_{\psi}$, the left-hand side of equality (3.14) equals $\left\|W_{\psi}[f]\right\|^{2}$ and the right-hand side equals $C_{\psi}\|f\|^{2}$.

\subsection{Wavelet Transformations Constructed by Unitary Irreducible Representations of Locally Compact Groups}

Rather than restricting ourselves to the particular case of left regular action of the $a x+b$ group representation on $\mathbb{L}_{2}(\mathbb{R})$, we will generalize by defining a wavelet transform given a Hilbert space $H$ and any unitary irreducible representation $g \mapsto \mho_{g}$ of any locally compact group $G$ in $H$. It is well-known that every locally compact group $G$ has a left-invariant Haar measure, which we denote by $\mu G$. A left-invariant Haar measure on $G$ is a Radon measure on $G$ such that $\mu_{G}(g E)=\mu_{G}(E)$ for all $g \in G$ and Borel sets $E$. It is uniquely determined up to a constant multiplicative factor, [26]. A nonzero vector $\psi$ in $H$ is said to be an admissible vector (or wavelet) if

$$
C_{\psi}=\int_{G} \frac{\left|\left(\mathcal{U}_{g} \psi, \psi\right)\right|^{2}}{(\psi, \psi)} d \mu_{G}(g)<\infty .
$$

Given an admissible vector $\psi$ and a unitary irreducible representation $\mathcal{U}$ of a locally compact group $G$ in a
Hilbert space $H$, the wavelet transform $\mathcal{W}_{\psi}: H \longrightarrow$ $\mathbb{L}_{2}(G)$ is defined by

$$
\mathcal{W}_{\psi}[f](g)=\left(\mathcal{U}_{g} \psi, f\right)_{H} .
$$

The next Theorem is well-known in mathematical physics [29] and was first formulated and proven by Grossmann et al. [19]. For a simple alternative and selfcontained proof, see [10] p. 20, which uses a topological version of Schur's lemma; for a proof, see [9] p. 86.

Theorem 3.2 (The Wavelet Reconstruction Theorem)

Let $U$ be irreducible; then, the wavelet transform given by (3.15) is a linear isometry (up to a constant) from the Hilbert space $H$ onto a closed subspace $\mathbb{C}_{K_{\psi}}^{G}$ of $\mathbb{L}_{2}(G, d \mu)$ :

$$
\left\|W_{\psi}[f]\right\|_{\mathbb{L}_{2}(G)}^{2}=C_{\psi}\|f\|^{2} .
$$

The space $\mathbb{C}_{K_{\psi}}^{G}$ is the unique functional Hilbert space with reproducing kernel $K_{\psi}\left(g, g^{\prime}\right)=\frac{1}{C_{\psi}}\left(U_{g} \psi\right.$, $\left.\boldsymbol{U}_{g^{\prime}} \psi\right)$. The corresponding orthogonal projection $\mathbb{P}_{\psi}$ : $\mathbb{L}_{2}(G, d \mu) \longrightarrow \mathbb{C}_{K_{\psi}}^{G}$ is given by

$$
\begin{gathered}
\left(\mathbb{P}_{\psi} \Phi\right)(g)=\int_{G} K_{\psi}\left(g, g^{\prime}\right) \Phi\left(g^{\prime}\right) d \mu_{G}\left(g^{\prime}\right) \\
\Phi \in \mathbb{L}_{2}(G, d \mu) .
\end{gathered}
$$

Furthermore, $\mathscr{W}_{\psi}$ intertwines the representation $\mathcal{U}$ and the left regular representation $\mathscr{L}$ (given by $\mathscr{L}_{g}(\Phi)=$ $\left.\left(h \mapsto \Phi\left(g^{-1} h\right)\right)\right)$ on $\mathbb{L}_{2}(G)$, that is $\mathcal{W}_{\psi} \mathcal{U}_{g}=\mathscr{L}_{g} \mathcal{W}_{\psi}$ for all $g \in G^{\prime \prime}$.

Of course, we would like to apply Theorem 3.2 to the wavelet transformation that maps an image to its orientation score (see (1.2)), since this would imply that the reconstruction of an image from its orientation score is perfectly well-posed in the sense that (just like Fourier transform) the quadratic norm is preserved. It follows by the next lemma that we are not allowed to apply Theorem 3.2 to our case. Therefore, in Section 4 we generalize the standard wavelet theory where irreducibility is neither a requirement nor replaced by a requirement.

Lemma 3.1. The left-regular action $U$ of the Euclidean motion group in $\mathbb{L}_{2}\left(\mathbb{R}^{2}\right)$, given by (2.6), is a reducible representation.

Proof. Consider the set of $\mathbb{L}_{2}$ functions whose Fourier transforms have a support inside a given disk around the origin with radius, say $\rho>0$; i.e., $\mathbb{L}_{2}^{\rho}\left(\mathbb{R}^{2}\right)$ : = $\left\{f \in \mathbb{L}_{2}\left(\mathbb{R}^{2}\right) \mid \operatorname{supp}(\mathscr{F}[f]) \subset B_{0, \rho}\right\}$; then obviously this is a non-trivial vector-space unequal $\mathbb{L}_{2}\left(\mathbb{R}^{2}\right)$, which is 


\begin{tabular}{|c|c|c|c|c|c|c|c|c|c|c|c|c|c|c|c|c|c|c|}
\hline 0 & $-\frac{1}{3}$ & 0 & \multirow{3}{*}{+} & 0 & 1 & 0 & \multirow[b]{2}{*}{+} & 0 & $-\frac{1}{3}$ & 0 & \multirow{3}{*}{+} & 0 & $-\frac{1}{3}$ & 0 & \multirow{3}{*}{$=$} & 0 & 0 & 0 \\
\hline$-\frac{1}{3}$ & 1 & 1 & & $-\frac{1}{3}$ & 1 & $-\frac{1}{3}$ & & 1 & 1 & $-\frac{1}{3}$ & & $-\frac{1}{3}$ & 1 & $-\frac{1}{3}$ & & 0 & 4 & 0 \\
\hline 0 & $-\frac{1}{3}$ & 0 & & 0 & $-\frac{1}{3}$ & 0 & & 0 & $-\frac{1}{3}$ & 0 & & 0 & 1 & 0 & & 0 & 0 & 0 \\
\hline
\end{tabular}

Fig. 3. Integrating the rotated kernel $\psi$ (left) over four discrete orientations gives a discrete spike $\delta$.

invariant under $u$, which directly follows by $\mathscr{F}\left[u_{g} \psi\right]=e^{i \boldsymbol{\omega} \cdot \mathbf{b}} \mathscr{R}_{\theta} \mathscr{F}[\psi]$ for all $\psi \in \mathbb{L}_{2}^{\rho}\left(\mathbb{R}^{2}\right)$.

Analogously to the example in Section 3.1, where we had to take an extra semi-direct product with $O(1)$, we could consider the similitude group $\operatorname{SIM}(2)=\mathbb{R}^{2} \times_{\tau}$ $\mathbb{T} \times D(1)$ with representation

$$
\begin{gathered}
\mathscr{V}_{\mathbf{b}, e^{i \theta}, a} \psi(\mathbf{x})=\frac{1}{\sqrt{a}} \psi\left(\frac{R_{\theta}^{-1}(\mathbf{x}-\mathbf{b})}{a}\right), \\
a>0, \quad \theta \in[0,2 \pi), \quad \mathbf{b} \in \mathbb{R}^{2},
\end{gathered}
$$

which is irreducible; for proof, see [23] pp. 51-52. This brings us within the standard wavelet framework of (and in particular to 2D Gabor wavelets, [22], or Cauchy-wavelets [3]) in 2D-image analysis. But from the implementation/practical point of view, we do not want to consider multiple scales, but stick to a single one. This coincides with Euclidean coherent states from mathematical physics, [20], which should not be mistaken for the more familiar Euclidean coherent states constructed from the irreducible ${ }^{5}$ (representations of the Euclidean motion group onto $\mathbb{L}_{2}\left(S^{1}\right)$ given by

$$
\begin{gathered}
\left(\mathscr{V}_{e^{i \theta}, \mathbf{b}}^{p} \psi\right)\left(e^{i \alpha}\right)=e^{i p\left(b^{1} \cos \alpha+b^{2} \sin \alpha\right)} \psi\left(e^{i(\alpha-\theta)}\right), \\
p>0, \quad \psi \in \mathbb{L}_{2}\left(S^{1}\right)
\end{gathered}
$$

(see [2] p. 219-220). Further, we notice that the generalization of the wavelet reconstruction theorem to reducible representations of quotient groups [29], inducing vector coherent states rather than coherent states, is also too restrictive to be applied to our case, as there is no (finite dimensional) dilation invariant subspace in $\mathbb{L}_{2}\left(\mathbb{R}^{2}\right)$.

From an image analysis point of view, omitting the dilation group poses an important question. For example, in scale space theory [13], it is a well-known problem that the reconstruction of a sharp image $f$ from its (e.g., Gaussian) blurred version $f * G_{s}$ is extremely illposed. Is it possible to get around this ill-posedness by considering all rotated versions of linear combinations

\footnotetext{
5 They are in fact, up to equivalence, the only irreducible representations of the Euclidean Motion group, cf. [27].
}

of Gaussian derivatives $f *\left(\partial_{x}\right)^{p}\left(\partial_{y}\right)^{q} G_{s}$ ? Before we give an affirmative answer to this question and deal with the issue of well-posed reconstruction of images from orientation scores, we give an illustration by means of an extremely simplified discrete example, where reconstruction is done by integration over discrete orientations, rather than inverse convolution.

Example. Suppose we construct a discrete orientation score with only four orientations-up, down, left, and right - constructed with the following discrete oriented wavelet $\psi: \mathbb{Z} \times \mathbb{Z} \longrightarrow \mathbb{R}$, given by

$$
\begin{gathered}
\Psi\left[x^{1}, x^{2}\right] \\
=\left\{\begin{array}{l}
1 \text { if }\left(x^{1}, x^{2}\right) \in\{(0,0),(0,1)\} \\
-1 / 3 \text { if }\left(x^{1}, x^{2}\right) \in\{(0,1),(0,-1),(-1,0)\} \\
0 \text { else. }
\end{array}\right.
\end{gathered}
$$

This wavelet detects a direction at a width of 2 pixels. Then reconstruction of the original discrete image $f: \mathbb{Z} \times \mathbb{Z} \longrightarrow \mathbb{R}$ from its orientation score is done by integration over all directions

$$
f\left[x^{1}, x^{2}\right]=\frac{1}{4} \sum_{k=1}^{4} U_{f}^{4}\left[x^{1}, x^{2}, e^{i \kappa \pi / 2}\right],
$$

since the right-hand side is in fact a convolution with a discrete $\delta$ spike (see Fig. 3).

\section{A FUNCTIONAL HILBERT SPACE APPROACH TO WAVELET THEORY}

In this section, we will put the theory of wavelets into a more generic framework. First we construct unitary maps from a Hilbert space $H$ into a functional Hilbert space $\mathbb{C}_{K}^{\square}$, which is a vector subspace of $\mathbb{C}^{\square}$, the vector space of all complex valued functions on a set $\square$ (not necessarily a group). In Subsection 4.2, we consider a special case and obtain a generalization of the standard wavelet theory for affine groups. The main strength of this generalization is that we will not assume the representation to be irreducible, which by Lemma 3.1 is necessary for our application.

Recall from Section 2 that a functional Hilbert space is a Hilbert space such that point evaluation is continu- 
ous, so by the Riesz representation theorem there exists a set $\left\{K_{m} \mid m \in \mathbb{}\right\}$ with

$$
\left(K_{m}, f\right)_{H}=f(m),
$$

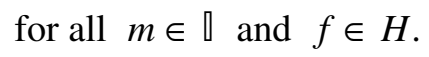

The span of the set $\left\{K_{m} \mid m \in \llbracket\right\}$ is dense in the functional Hilbert space. Indeed, if $f \in H$ is orthogonal to all $K_{m}$, then $f=0$ on $\mathbb{~ . ~}$

Then define $K\left(m, m^{\prime}\right)=K_{m^{\prime}}(m)=\left(K_{m}, K_{m^{\prime}}\right)_{H}$, for $m$, $m^{\prime} \in \mathbb{Z}$. $K$ is called the reproducing kernel and it is a

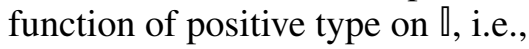

$$
\sum_{i=1}^{n} \sum_{j=1}^{n} K\left(m_{i}, m_{j}\right) \bar{c}_{i} c_{j} \geq 0,
$$

for all $n \in \mathbb{N}, \quad c_{1}, \ldots, c_{n} \in \mathbb{C}, \quad m_{1}, \ldots, m_{n} \in \mathbb{\square}$.

So to every functional Hilbert space there belongs a reproducing kernel, which is a function of positive type. Conversely, as Aronszajn pointed out in his paper [4], a function $K$ of positive type on a set $\square$ uniquely induces a functional Hilbert space consisting of functions on ए with reproducing kernel $K$. We denote this space by $\mathbb{C}_{K}^{\natural}$. Before briefly discussing the construction of this space, we give an illustrative example.

Example. Consider the first order Sobolev space $\mathbb{H}^{1}(\mathbb{R}), \mathbb{\square}=\mathbb{R}$, with

$$
(f, g)_{\mathbb{H}^{1}(\mathbb{R})}=(f, g)_{\mathbb{\bigsqcup}_{2}(\mathbb{R})}+\left(f^{\prime}, g^{\prime}\right)_{\mathbb{\bigsqcup}_{2}(\mathbb{R})},
$$

where $f^{\prime}$ and $g^{\prime}$ are the generalized first order derivatives of resp. $f$ and $g$. Then, $\delta_{a}$ is a bounded (i.e., continuous) linear functional on $\mathbb{T}^{1}(\mathbb{R})$. This follows by the estimate

$$
\begin{aligned}
&|f(a)|^{2}=\int_{a-1}^{a}\{(x-(a-1)) \overline{f(x)} f(x)\}^{\prime} d x \\
&=\int_{a-1}^{a}\left\{|f(x)|^{2}+(x-(a-1))\right. \\
&\left.\times\left[\overline{f^{\prime}(x)} f(x)+\overline{f(x)} f^{\prime}(x)\right]\right\} d x \\
& \leq \int_{a-1}^{a}\left\{|f(x)|^{2}+2\left|f^{\prime}(x)\right||f(x)|\right\} d x \\
& \leq \int_{a-1}^{a}\left\{2|f(x)|^{2}+\left|f^{\prime}(x)\right|^{2}\right\} d x \leq 2\|f\|_{\mathbb{H}^{1}(\mathbb{R})}^{2}
\end{aligned}
$$

So, by the Riesz representation theorem, the reproducing kernel exists and by Fourier transformation it follows that the reproducing kernel is given by

$$
\begin{gathered}
K(a, x)=\mathscr{F}^{-1}\left[e^{i \omega a} \frac{1}{1+|\omega|^{2}}\right](x)=(1 / 2) e^{-|x-a|}, \\
a, x \in \mathbb{R}
\end{gathered}
$$

and $\left\|\delta_{a}\right\|_{\mathbb{H}^{-1}(\mathbb{R})}=\left\|K_{a}\right\|_{\mathbb{H}^{1}(\mathbb{R})}=(1 / 2) \sqrt{2}$. So if $K$ is given by (4.19), then $\mathbb{C}_{K}^{\mathbb{Q}=\mathbb{R}}=\mathbb{H}^{1}(\mathbb{R})$.

Next we give a brief overview of how to construct $\mathbb{C}_{K}^{\natural}$ given the index set $\square$ and the function of positive type $K: \rrbracket \times \rrbracket \longrightarrow \mathbb{C}$. The span $\left\langle\left\{K_{m} \mid m \in \llbracket\right\}\right\rangle$ is a preHilbert space with respect to the inner product

$$
\left(\sum_{i=1}^{l} \alpha^{i} K_{m_{i}}, \sum_{i=1}^{n} \beta^{j} K_{m_{j}}\right)=\sum_{i=1}^{n} \sum_{j=1}^{l} \overline{\alpha^{i}} \beta^{j} K\left(m_{i}, m_{j}\right) .
$$

By taking the completion we get $\mathbb{C}_{K}^{\natural}$. The norm $\|\Phi\|=\sqrt{(\Phi, \Phi)}$ on the space $\mathbb{C}_{K}^{\natural}$ is given by

$$
\|\Phi\|_{\mathbb{C}_{K}^{\square}}=\sup \left\{\frac{\left|(\Phi, G)_{\mathbb{C}_{K}^{\square}}\right|^{2}}{\|G\|_{\mathbb{C}_{K}^{\square}}^{2}} \mid G \in\left\langle\left\{K_{m} \mid m \in \llbracket\right\}\right\rangle\right\}
$$

$$
\begin{gathered}
=\sup \left\{\left|\sum_{j=1}^{l} \alpha_{j} \overline{\Phi\left(m_{j}\right)}\right|^{2}\left(\sum_{k, j=1}^{l} \overline{\alpha_{k}} \alpha_{j} K\left(m_{k}, m_{j}\right)\right)^{-1}(4.20)\right. \\
\left.\mid l \in \mathbb{N}, \alpha_{j} \in \mathbb{C}, m_{j} \in \mathbb{Q}, \sum_{k, j=1}^{l} \overline{\alpha_{k}} \alpha_{j} K\left(m_{k}, m_{j}\right) \neq 0\right\}
\end{gathered}
$$

(see [24] Lemma 1.7, p. 31). We highly stress that this abstract construction of $\mathbb{C}_{K}^{0}$ may not be satisfactory in many cases. Recall for example the example of the first order Sobolev space $\mathbb{H}^{1}(\mathbb{R})$. In this case, the norm is both given by (4.18) and by (4.20), but clearly (4.18) is a much more tangible characterization of the norm than (4.20).

\subsection{The Construction of a Unitary Map from $H$ to $\mathbb{C}_{K}^{\square}$}

Let $V=\left\{\phi_{m} \mid m \in \llbracket\right\}$ be a subset of $H$ such that its linear span is dense in $H$. Define the function $K: \llbracket \times \rrbracket \longrightarrow$ $\mathbb{C}$ by $K\left(m, m^{\prime}\right)=\left(\phi_{m}, \phi_{m}^{\prime}\right)_{H}$. Given such a function, we define the function $K_{m}: \mathbb{} \longrightarrow \mathbb{C}$ as $K_{m}\left(m^{\prime}\right)=K\left(m, m^{\prime}\right)$. In earlier work [10], we obtained the following fundamental result: 
Theorem 4.3. If the span of $V=\left\{\phi_{m} \mid m \in \llbracket\right\}$ is dense in $H$, then the transform $\mathcal{W}: H \mapsto \mathbb{C}_{K}^{\rrbracket}$ defined by

$$
(\mathcal{W}[f])(m)=\left(\phi_{m}, f\right)_{H}
$$

is a unitary mapping; i.e., $\|\mathcal{W}[f]\|_{\mathbb{C}_{K}^{0}}=\|f\|_{H}$.

Proof. See Appendix B.

From the applied mathematical point of view, this result in itself may not be satisfactory, since the norm

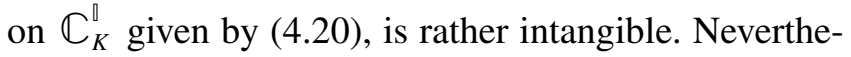
less, in some special cases, this norm has a simpler explicit form.

\section{Special Cases:}

- Wavelet transforms: Let $\square=G$ for some locally compact group $G$. Let $\psi \in H$ (the wavelet). Let $g \mapsto \mathscr{R}_{g}$ fig be a representation of $G$ into $H$ such that

$$
V_{\psi}=\left\{\mathscr{R}_{g} \psi \mid g \in G\right\}
$$

is dense in $H$ and $K\left(g, g^{\prime}\right)=\left(\mathscr{R}_{g} \psi, R_{g^{\prime}} \psi\right)_{H}$; then by Theorem 4.3, the generalized Wavelet Transform given by

$$
\left(\mathcal{W}_{\psi}[f]\right)(g)=\left(\mathscr{R}_{g} \psi, f\right)_{H}
$$

is a unitary mapping from $H$ onto $\mathbb{C}_{K}^{G}$. Notice that it immediately follows by the Cauchy-Schwarz inequality that $\delta_{g}$ is indeed continuous on $\mathbb{C}_{K}^{G}$ :

$$
\begin{gathered}
\left\langle\delta_{g}, \mathcal{W}_{\psi}[f]\right\rangle=\left|\mathcal{W}_{\psi}[f](g)\right| \leq\left\|\mathcal{U}_{g} \psi\right\|_{H}\|f\|_{H}, \\
\text { for all } g \in G, \quad f \in H .
\end{gathered}
$$

- Discrete frames: Let $\left\{\psi_{i} \mid i \in N\right\}$ be a frame within some Hilbert space $H$ with frame bounds $m>0$ and $M>0$, i.e.,

$$
m\|f\|^{2} \leq \sum_{j=1}^{\infty}\left|\left(\psi_{i}, f\right)\right|^{2} \leq M\|f\|^{2},
$$

for all $f \in H=\overline{\operatorname{span}\left\{\psi_{i}\right\}}$. The orthogonal projection onto $H$ is given by

$$
\mathbb{P}_{H} f=\sum_{j=1}^{\infty}\left(\psi_{j} f\right) \tilde{\psi}_{j}=\sum_{j=1}^{\infty}\left(\tilde{\psi}_{j}, f\right) \psi_{j}, f \in \hat{H},
$$

in which $\tilde{\psi}_{j}=\mathscr{A}^{-1} \psi_{j}$ is the reciprocal frame, where $\mathscr{A}^{-1}$ is the bounded inverse ${ }^{6}$ of the bounded operator ${ }^{7} \mathscr{A} \psi=$ $\sum_{i \in \mathbb{N}}\left(\psi_{i}, \psi\right)_{H} \psi_{i}$. Now set $\mathbb{\|}=\mathbb{N}, V=\left\{\psi_{i}\right\}, K(i, j)=\left(\psi_{i}\right.$, $\left.\psi_{j}\right)$, and $H=\overline{\operatorname{span}\left\{\psi_{i}\right\}}$; then from Theorem 4.3 we

\footnotetext{
${ }^{6}$ This directly follows from the frame-bound $m>0$ in (4.24) and the Lax-Milgram theorem.

7 This directly follows by the frame bound $M>0$ in (4.24).
}

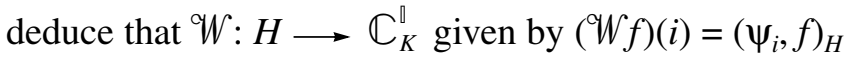
is a unitary mapping.

If $\left\{\psi_{i}\right\}$ are linear independent, the Gramm-matrix $g_{i j}$ is invertible and it follows that $\tilde{\psi}_{i}$ equals the reciprocal basis $\tilde{\psi}^{j}=g^{j k} \psi_{k}$, where $g^{j k}$ are the matrix elements of the inverse of the Gramm-matrix. In this case, $\mathbb{C}_{K}^{\mathbb{1}}$ is a subspace of $\ell_{2}(\mathbb{N})=\left\{\mathbf{a}=\left.\left(a_{k}\right)_{k \in \mathbb{N}} \in \mathbb{C}^{N}\left|\sum_{k \in \mathbb{N}}\right| a_{k}\right|^{2}<\infty\right\}$ equipped with inner product

$$
\begin{gathered}
(\mathbf{a}, \mathbf{b})=\sum_{n \in \mathbb{N}}\left\{\sum_{k \in \mathbb{N}} \overline{g^{k n} a_{k}}\right\} b_{n}, \\
\mathbf{a}=\left\{a_{k}\right\}_{k \in \mathbb{N}}, \quad \mathbf{b}=\left\{b_{n}\right\}_{n \in \mathbb{N}} \in \ell_{2}(\mathbb{N}) .
\end{gathered}
$$

The reproducing kernel is given by $K_{m}(k)=K(m$, $k)=g_{m k}=\left(\psi_{m}, \psi_{k}\right)$ :

$$
\begin{gathered}
\left(K_{m}, \mathbf{b}\right)=\sum_{n \in \mathbb{N}}\left\{\sum_{k \in \mathbb{N}} \overline{g^{k n} g_{m k}}\right\} b_{n}=\sum_{n} \delta_{m}^{n} b_{n}=b_{m}, \\
\text { for all } \mathbf{b} \in l_{2}(\mathbb{N}), \quad m \in \mathbb{N} .
\end{gathered}
$$

If $m=M=1$ then $\left\{\psi_{i}\right\}_{i=1}^{\infty}$ is an orthogonal base (see [8] p. 57) and $\mathscr{G}=\mathscr{G}^{-1}=I$, and thereby $\mathbb{C}_{K}^{\mathbb{1}}=\ell_{2}(\mathbb{N})$ equipped with a standard $\ell_{2}$-inner product.

If $\left\{\psi_{i}\right\}_{i=1}^{\infty}$ is a Riesz basis in $H$, i.e., there exist $c$, $C>0$ such that

$$
\begin{gathered}
c\|\mathbf{a}\|_{\ell_{2}} \leq\left\|\sum_{n \in \mathbb{N}} a_{n} \psi_{n}\right\|^{2} \leq C\|\mathbf{a}\|_{\ell_{2}}, \\
\text { for all } \mathbf{a} \in \ell_{2}(\mathbb{N}),
\end{gathered}
$$

then there exists a bounded invertible linear operator $U$ on $H$ such that $\left\{U \psi_{n}\right\}$ is an orthonormal basis in $H$. Then it follows by $\left(\psi_{i}, f\right)=\left(U \psi_{i},\left(U^{*}\right)^{-1} f\right)$ and the previous case, that $\mathbb{C}_{K}^{\natural}=\ell_{2}(\mathbb{N})$ equipped with inner product: $(\mathbf{a}, \mathbf{b})_{\mathbb{C}_{K}^{0}}=\sum_{n \in \mathbb{N}} \overline{u_{n}^{k} a_{k}} u_{n}^{k} b_{l}$, where $u_{n}^{k}=\left(\psi^{k}, \mathcal{U}^{*} \psi_{n}\right)$.

Next, we give an explicit characterization of $\mathbb{C}_{K}^{G}$ in the case $G=\mathbb{R}^{d} \times_{\tau} T$ and $\mathcal{U}$ the left-regular action of $G$ onto $H=\mathbb{L}_{2}\left(\mathbb{R}^{d}\right)$ and thereby formulate a generalization of the wavelet reconstruction theorem for affine groups.

\subsection{Generalization of Wavelet Reconstruction Theorem for Affine Groups}

Inspired by Section 3.2 , we call $\psi \in \mathbb{L}_{2}\left(\mathbb{R}^{d}\right)$ an admissible wavelet if 


$$
\begin{gathered}
0<M_{\psi}:=(2 \pi)^{d / 2} \int_{T}\left|\frac{\mathscr{F}_{f}\left[\mathscr{R}_{t} \psi\right]}{\sqrt{\operatorname{det} \tau(t)}}\right|^{2} d \mu_{T}(t)<\infty \\
\text { a.e. on } \mathbb{R}^{d},
\end{gathered}
$$

where we recall that $\mathscr{R}_{t}$ is given by (2.7) and where we notice that the function $M_{\psi}$ for the special case $G=$
$\mathbb{R}^{2} \times \mathbb{\mathbb { T }}$ indeed corresponds to definition (2.9) in Section 2 .

Lemma 4.2 Let $\psi$ be an admissible Wavelet. Then the span of $V_{\psi}$ (for definition see (4.22)) is dense in $\mathbb{L}_{2}\left(\mathbb{R}^{d}\right)$, i.e., $\overline{\left\langle V_{\psi}\right\rangle}=\mathbb{L}_{2}\left(\mathbb{R}^{d}\right)$.

\section{Proof.}

$$
\begin{aligned}
& f \in\left\langle V_{\psi}\right\rangle^{\perp} \Leftrightarrow \forall_{\mathbf{b} \in \mathbb{R}^{d}} \forall_{t \in T}\left(\mathcal{U}_{\mathbf{b}, t} \psi, f\right)_{\mathbb{I}_{2}\left(\mathbb{R}^{d}\right)}=0 \\
& \Leftrightarrow \forall_{\mathbf{b} \in \mathbb{R}^{d}} \forall_{t \in T}\left((\mathbf{b}, t) \mapsto \mathscr{F}^{-1}\left[\left[\mathscr{F}_{\mathscr{F}} \mathscr{R}_{t} \psi\right][\mathscr{F} f]\right](\mathbf{b})\right)=0 \\
& \Rightarrow\left((\boldsymbol{\omega}, t) \mapsto \mathscr{F}_{\mathscr{R}} \psi(\boldsymbol{\omega}) \mathscr{F} f(\boldsymbol{\omega})\right)=0 \quad \text { a.e. on } \mathbb{R}^{d} \times_{\tau} T \\
& \Rightarrow\left((\boldsymbol{\omega}, t) \mapsto\left|\mathscr{F}_{\mathscr{R}_{t}} \psi(\boldsymbol{\omega}) \mathscr{F} f(\boldsymbol{\omega})\right|^{2}\right)=0 \quad \text { a.e. on } \mathbb{R}^{d} \times_{\tau} T
\end{aligned}
$$

Let $f \in\left\langle V_{\psi}\right\rangle^{\perp}$. Then

$$
\begin{gathered}
M_{\psi}(\boldsymbol{\omega})|(\mathscr{F} f)(\boldsymbol{\omega})|^{2} \\
=(2 \pi) \int_{T}^{d / 2}\left|\frac{\mathscr{F}\left[\mathscr{R}_{t} \psi\right](\boldsymbol{\omega}) \mathscr{F} f(\boldsymbol{\omega})}{\sqrt{\operatorname{det} \tau(t)}}\right|^{2} d \mu_{T}(t)=0 \\
\text { a.e. on } \mathbb{R}^{d} .
\end{gathered}
$$

Because $\psi$ is an admissible wavelet, $M_{\psi}>0$ a.e. $\Rightarrow$ $|\mathscr{F} f|^{2}=0 \Rightarrow f=0$.

Corollary 1. If the wavelet $\psi$ is admissible, then the corresponding wavelet transform $\mathcal{W}_{\psi}: \mathbb{L}_{2}\left(\mathbb{R}^{d}\right) \longrightarrow \mathbb{C}_{K}^{G}$ is unitary.

Proof. This follows from Lemma 4.2 and Theorem 4.3.

Define the linear operator $\mathscr{T}_{M_{\psi}}$ on $\mathbb{C}_{K}^{G}$ by

$$
\begin{gathered}
{\left[\mathscr{T}_{M_{\psi}}[\Phi]\right](\mathbf{b}, t)} \\
=\mathscr{F}^{-1}\left[\boldsymbol{\omega} \mapsto(2 \pi)^{-d / 4} M_{\psi}^{-\frac{1}{2}}(\boldsymbol{\omega}) \mathscr{F}[\Phi(\cdot, t)](\boldsymbol{\omega})\right](\mathbf{b}) .
\end{gathered}
$$

Operator $\mathscr{T}_{M_{\psi}}$, is well-defined since $M_{\psi}>0$ a.e. on $\mathbb{R}^{d}$ and $M_{\psi}^{-\frac{1}{2}} \mathscr{F}[\Phi(\cdot, t)] \in \mathbb{L}_{2}\left(\mathbb{R}^{d}\right)$ for all $t \in T$.

Theorem 4.4. Let $G=\mathbb{R}^{d} \times_{\tau} T$. Let $\psi$ be an admissible wavelet. Then $\mathscr{T}_{M_{\psi}} \Phi \in \mathbb{L}_{2}\left(G, d \mu_{G}(g)\right)$ for all $\Phi \in$ $\mathbb{C}_{K}^{G}$. Therefore, $(\cdot, \cdot)_{M_{\psi}}:\left(\mathbb{C}_{K}^{G}\right)^{2} \longrightarrow \mathbb{C}$ defined by

$$
(\Phi, \Psi)_{M_{\psi}}=\left(\mathscr{T}_{M_{\psi}}[\Phi], \mathscr{T}_{M_{\psi}}[\Psi]\right)_{\mathbb{L}_{2}(G)},
$$

is an explicit characterization of the inner product on $\mathbb{C}_{K}^{G}$, which is the unique functional Hilbert space with reproducing kernel $K: G \times G \longrightarrow \mathbb{C}$ given by

$$
\begin{aligned}
& K(g, h)=\left(\mathcal{U}_{g} \psi, \mathcal{U}_{h} \psi\right)_{\mathbb{L}_{2}\left(\mathbb{R}^{2}\right)} \\
& =\left(\mathcal{U}_{h^{-1} g} \psi, \psi\right)_{\mathbb{E}_{2}\left(\mathbb{R}^{d}\right)}, g, h \in G .
\end{aligned}
$$

The Wavelet transformation $\mathcal{W}_{\psi}$ defined by

$$
\begin{gathered}
\mathscr{W}_{\psi}[f](\mathbf{b}, t)=\left(\mathcal{U}_{g} \psi, f\right)_{\mathbb{L}_{2}\left(\mathbb{R}^{2}\right)}, \\
f \in \mathbb{L}_{2}\left(\mathbb{R}^{d}\right), \quad g=(\mathbf{b}, t) \in \mathbb{R}^{d} \times{ }_{\tau} T,
\end{gathered}
$$

is a unitary mapping from $\mathbb{L}_{2}\left(\mathbb{R}^{d}\right)$ to $\mathbb{C}_{K}^{G}$.

The space $\mathbb{C}_{K}^{G}$ is a closed subspace of the Hilbert space $\mathbb{H}_{\psi} \otimes \mathbb{L}_{2}\left(T ; \frac{d \mu_{T}}{\operatorname{det}(\tau(t))}\right)$, where $\mathbb{H}_{\psi}=\{f \in$ $\left.\mathbb{L}_{2}\left(\mathbb{R}^{d}\right) \mid M_{\psi}^{-\frac{1}{2}} \mathscr{F}[f] \in \mathbb{L}_{2}\left(\mathbb{R}^{d}\right)\right\}$ is equipped with inner product

$$
(f, g)=\left(M_{\psi}^{-\frac{1}{2}} \mathscr{F} f, M_{\psi}^{-\frac{1}{2}} \mathscr{F} g\right)_{\mathbb{L}_{2}\left(\mathbb{R}^{d} ;(2 \pi)^{-d / 2} d x\right)} .
$$

The orthogonal projection $\mathbb{P}_{\psi}$, of $\mathbb{H}_{\psi} \otimes$ $\mathbb{L}_{2}\left(T ; \frac{d \mu_{T}}{\operatorname{det}(\tau(t))}\right)$, onto $\mathbb{C}_{K}^{G}$ is given by $\mathbb{P}_{\psi}[\Phi]=$ $(K(\cdot, g), \Phi)_{M_{\psi}}$.

Proof. See ${ }^{8}[10]$ p. $27-30$.

Remarks.

(1) Since $W_{\psi}: \mathbb{L}_{2}\left(\mathbb{R}^{d}\right) \longrightarrow \mathbb{C}_{K}^{G}$ is unitary, the inverse equals the adjoint and as a result image $f$ can be reconstructed from its orientation score $\mathcal{W}_{\psi}[f]$ by

\footnotetext{
${ }^{8}$ Here, the result is generalized to semi-direct products of a locally compact group $T$ and any commutative group $S$. Here, we only consider $S=\mathbb{R}^{d}$.
} 


$$
\begin{gathered}
f=\mathcal{W}_{\psi}^{*}\left[\mathcal{W}_{\psi}[f]\right] \\
=\mathscr{F}^{-1}\left[\boldsymbol{\omega} \mapsto \int_{T} \mathscr{F}_{T}\left[\mathcal{W}_{\psi}[f](\cdot, t)\right](\boldsymbol{\omega})\right. \\
\left.\times \mathscr{F}_{F}\left[\mathscr{R}_{t} \psi\right](\boldsymbol{\omega}) \frac{d \mu_{T}(t)}{|\operatorname{det}(\tau(t))|} M_{\psi}^{-1}(\boldsymbol{\omega})\right] .
\end{gathered}
$$

(2) If $M_{\psi}=(2 \pi)^{d / 2}$, then $\mathbb{C}_{K}^{\mathbb{R}^{d} \times_{\tau} T}$ is a closed subspace of $\mathbb{L}_{2}(G)=\mathbb{L}_{2}\left(\mathbb{R}^{d} \times{ }_{\tau} T\right)$. Then, as in the irreducible case, Theorem 3.2, the quadratic norm is preserved.

(3) It is easily verified that $\mathscr{F}\left(\mathscr{R}_{t} \psi\right)(\boldsymbol{\omega})=$ $\sqrt{\operatorname{det} \tau(t)}[\mathscr{F} \psi]\left(\tau(t)^{T} \boldsymbol{\omega}\right)$, for all $\boldsymbol{\omega} \in \mathbb{R}^{d}$ and $t \in T$. As a result, $\mathcal{M}_{\psi}$ can be rewritten as

$$
\begin{gathered}
M_{\psi}(\boldsymbol{\omega})=(2 \pi)^{\frac{d}{2}} \int_{T}\left|[\mathscr{F} \psi]\left((\tau(t))^{T} \boldsymbol{\omega}\right)\right|^{2} d \mu_{T}(t), \\
\boldsymbol{\omega} \in \mathbb{R}^{d} .
\end{gathered}
$$

From this expression (and the left invariance of Haar measure), we conclude that

$$
\begin{aligned}
& M_{\psi}\left((\tau(t))^{T} \boldsymbol{\omega}\right)=M_{\psi}(\boldsymbol{\omega}), \\
& \text { for all } \boldsymbol{\omega} \in \mathbb{R}^{d} \text { and } t \in T .
\end{aligned}
$$

Now assume that $t \mapsto(\tau(t))^{T} \boldsymbol{\omega}$ acts transitively on $\mathbb{R}^{d}$; then it follows by (4.30) that $M_{\psi}$ is constant and the space $\mathbb{C}_{K}^{G}, G=\mathbb{R}^{d} \times_{\tau} T$, is a closed subspace of $\mathbb{L}_{2}(G)$.

Example in the case of $T=\mathscr{D}(1) \times S O(2)$, which acts transitively on $\mathbb{R}^{2}$, we get the similitude group $G=$ $\mathbb{R}^{2} \times{ }_{\tau} T=\mathbb{R}^{2} \times \mathscr{D}(1) \times S O(2)$. So the functional Hilbert space $\mathbb{C}_{K}^{G}$ is indeed a closed subspace of $\mathbb{L}_{2}(G)$ and the corresponding wavelet transform preserves the quadratic norm.

(4) If $T$ is a compact group (for example $\mathbb{T}$ ) then it follows by interchanging the order of summation and integration (Fubini) that $M_{\psi} \in \mathbb{L}_{1}\left(\mathbb{R}^{d}\right)$. As a result, the desirable case $M_{\psi}=(2 \pi)^{d / 2}$ cannot be achieved.

(5) The transform $\mathcal{W}_{\psi}$ can be rewritten as

$$
\begin{gathered}
\left(\mathscr{W}_{\psi} f\right)(\mathbf{b}, t) \\
=\left(\mathscr{U}_{(\mathbf{b}, t)} \psi, f\right)=\left(\mathscr{F}_{\mathbf{F}} \mathscr{R}_{t} \psi, \mathscr{F}_{F}\right) \\
=\sqrt{|\operatorname{det} \tau(t)|} \int_{\mathbb{R}^{d}} \mathrm{e}^{\mathrm{i}(\omega, \mathbf{b})} \overline{\left(\mathscr{R}_{(\tau(t))^{-T}} \mathscr{F} \psi\right)(\omega)} \mathscr{F} f(\omega) \mathrm{d} \omega \\
=\sqrt{(2 \pi)^{d}|\operatorname{det} \tau(t)|}\left(\mathscr{F} *\left(\sqrt{\mathscr{R}_{(\tau(t))^{-T}} \mathscr{F}_{\mathcal{F}} \psi} \mathscr{F}_{F} f\right)\right)(\mathbf{b}),
\end{gathered}
$$

This provides the backbone of the proof of Theorem 4.4, which is the following Parceval equality:

$$
\begin{gathered}
\left\|W_{\psi} f\right\|_{M_{\psi}}^{2} \\
=\int_{\mathbb{R}^{d}}(2 \pi)^{-\frac{d}{2}} \int_{T}\left|\left(\mathscr{F} W_{\psi} f\right)(\omega, t)\right|^{2} \frac{\mathrm{d} \mu_{T}(t)}{|\operatorname{det}(\tau(t))|} \frac{\mathrm{d} \omega}{M_{\psi}(\omega)} \\
=\int_{\mathbb{R}^{d}}(2 \pi)^{\frac{d}{2}} \int_{T}|\mathscr{F} f(\omega)|^{2}\left|\mathscr{F} \psi\left((\tau(t))^{T} \omega\right)\right|^{2} \mathrm{~d} \mu_{T}(t) \frac{\mathrm{d} \omega}{M_{\psi}(\omega)} \\
=\int_{\mathbb{R}^{d}}(2 \pi)^{\frac{d}{2}} \frac{|\mathscr{F} f(\omega)|^{2}}{M_{\psi}(\omega)} \int_{T}\left|\mathscr{F} \psi\left((\tau(t))^{T} \omega\right)\right|^{2} \mathrm{~d} \mu_{T}(t) \mathrm{d} \omega \\
=\int_{\mathbb{R}^{d}}|\mathscr{F} f(\omega)|^{2} \mathrm{~d} \omega=\|f\|_{\mathbb{L}_{2}\left(\mathbb{R}^{d}\right)}^{2}
\end{gathered}
$$

that holds for all $f \in \mathbb{L}_{2}\left(\mathbb{R}^{d}\right)$.

Definition 4.1. The inner product on $\mathbb{H}_{\psi} \otimes$ $\mathbb{L}_{2}\left(T ; \frac{\mu_{T}}{\operatorname{det}(\tau(t))}\right)$ induces a norm $\|\cdot\|_{M_{\psi}}: \mathbb{H}_{\Psi} \otimes$ $\mathbb{L}_{2}\left(T ; \frac{\mu_{T}}{\operatorname{det}(\tau(t))}\right) \rightarrow \mathbb{R}^{+}$, which is given by

$$
\begin{gathered}
\|\Phi\|_{M_{\psi}}=\sqrt{(\Phi, \Phi)_{M_{\psi}}} \\
=\iint_{\mathbb{R}^{2} T}|\mathscr{F}[\Phi(\cdot, t)](\boldsymbol{\omega})|^{2}(2 \pi)^{-d / 2} M_{\psi}^{-1}(\boldsymbol{\omega}) \frac{d \mu_{T}(t)}{\operatorname{det} \tau(t)} d \boldsymbol{\omega},
\end{gathered}
$$

which we will call the $M_{\psi}$-norm.

Theorem 4.4 has the following important consequence for our application of orientation scores in image analysis:

Corollary 2. The space of orientation scores is a reproducing kernel Hilbert space $\mathbb{C}_{K}^{\mathbb{R}^{2} \times \mathbb{T}}$ which is a closed subspace of $\mathbb{H}_{\psi} \otimes \mathbb{L}_{2}\left(\mathbb{\mathbb { N }} ; \frac{\mu_{T}}{\operatorname{det}(\tau(t))}\right)$, which is a vector subspace ${ }^{9}$ of $\mathbb{L}_{2}(G)$. The inner product on $\mathbb{C}_{K}^{\mathbb{R}^{2} \times \mathbb{T}}$ is given by (4.26) and is explicitly characterized by means of the function $M_{\psi}$ given in (2.9). The wavelet transformation which maps an image $f \in \mathbb{L}_{2}\left(\mathbb{R}^{2}\right)$ onto its orientation score $U_{f} \in \mathbb{C}_{K}^{\mathbb{R}^{2} \times \mathbb{T}}$ is a unitary mapping: $\|f\|_{\mathbb{L}_{2}\left(\mathbb{R}^{2}\right)}^{2}=\left\|U_{f}\right\|_{M_{\psi}}^{2}$.

\footnotetext{
${ }^{9}$ I.e., a subspace like a vector space, but equipped with a different norm.
} 
As a result, the image $f$ can be reconstructed from its orientation score $U_{f}=\mathcal{W}_{\psi}[f]$ by means of the adjoint wavelet transformation $\mathcal{W}_{\psi}^{*}$ :

$$
\begin{gathered}
f=\mathscr{W}_{\psi}^{*} W_{\psi}[f] \\
=\mathscr{F}^{-1}\left[\boldsymbol{\omega} \mapsto \int_{0}^{2 \pi} \mathscr{F}\left[U_{f}\left(\cdot, e^{i \theta}\right)\right](\boldsymbol{\omega})\right. \\
\left.\times \mathscr{F}_{\mathscr{F}}\left[\mathscr{R}_{e^{i \theta}} \psi\right](\boldsymbol{\omega}) d \theta M_{\psi}^{-1}(\boldsymbol{\omega})\right] .
\end{gathered}
$$

Proof. Take $d=2, T=\mathbb{T}$, and $\tau: \mathbb{T} \longrightarrow \operatorname{Aut}\left(\mathbb{R}^{2}\right)$ given by $\tau\left(e^{i \theta}\right) \mathbf{x}=R_{\theta} \mathbf{x}$ in Theorem 4.4.

4.2.1. The discrete analogue. Since the general theory deals with $G=T \times \mathbb{R}^{d}$, with $T$ locally compact, we may as well choose $T$ a finite rotation group (equipped with discrete topology) which is locally compact. So consider $G=\mathbb{T}_{N} \times \mathbb{R}^{2}$, where

$$
\mathbb{T}_{N}=\left\{e^{i k \Delta} \mid k \in\{0,1, \ldots, N-1\}, \Delta=\frac{2 \pi}{N}\right\},
$$

$$
\text { for } N \in \mathbb{N} \text {. }
$$

The discrete version of Corollary 2 is then straightforward, where we notice that the discrete orientation score $U_{f}^{N}\left(\mathbf{b}, e^{i k \Delta}\right)$ of an image $f \in \mathbb{L}_{2}\left(\mathbb{R}^{2}\right)$ is given by

$$
\begin{gathered}
U_{f}^{N}\left(\mathbf{b}, e^{i k \Delta}\right)=\left(\mathscr{T}_{\mathbf{b}} \mathscr{R}_{k \Delta} \psi, f\right)_{\mathbb{L}_{2}\left(\mathbb{R}^{2}\right)}, \\
k \in\{0,1, \ldots, N-1\}, \quad \Delta=\frac{2 \pi}{N},
\end{gathered}
$$

and the discrete version of the function $M_{\psi}$ is $M_{\psi}(\boldsymbol{\omega})=$ $\frac{1}{N} \sum_{k=0}^{N-1}\left|\mathscr{F}_{(}\left(\mathscr{R}_{k \Delta} \psi\right)(\boldsymbol{\omega})\right|^{2}$.

\section{STABILITY \\ OF THE IMAGE RECONSTRUCTION}

The usual way to quantify the well-posedness/stability of an invertible linear transformation $A: V \longrightarrow W$ from a normed space $\left(V,\|\cdot\|_{V}\right)$ to a normed space $(W$, $\left.\|\cdot\|_{W}\right)$ is by means of the condition number

$$
\begin{gathered}
\operatorname{cond}(A)=\left\|A^{-1}\right\|\|A\| \\
=\left(\sup _{\mathbf{x} \in V} \frac{\|\mathbf{x}\|_{V}}{\|A \mathbf{x}\|_{W}}\right)\left(\sup _{\mathbf{x} \in V} \frac{\|A \mathbf{x}\|_{W}}{\|\mathbf{x}\|_{V}}\right) \geq 1 .
\end{gathered}
$$

The closer it approximates 1 , the more stable the operator and its inverse are. Notice that the condition number depends on the norms imposed on $V$ and $W$. We want to apply this general concept to the wavelet trans- formation which maps image $f$ to its orientation score ${ }^{10}$ $U_{f}$. In the previous section, we considered the wavelet transform as a unitary mapping from the space $\mathbb{L}_{2}\left(\mathbb{R}^{2}\right)$ to the space $\mathbb{C}_{K}^{G}$ equipped with $\mathbb{L}_{2}$-norm and $M_{\psi}$-norm respectively. Consequently, with these chosen norms, the condition number becomes 1 . However, from a practical and numerical point of view it is much more reasonable to impose the $\mathbb{L}_{2}(G)$-norm on the orientation score, since this norm does not depend on the wavelet $\psi$ and is in general not sensitive to noise. Moreover, it seems consistent, with the $\mathbb{L}_{2}$-norm imposed on the space of images ${ }^{11}$.

Furthermore, we assume that our images $f$ are bandlimited ${ }^{12}$, i.e., the support of their Fourier transform is bounded, by, e.g., a sphere with radius $\boldsymbol{\rho}$. The space of these images is given by

$$
\begin{gathered}
\mathbb{L}_{2}^{\boldsymbol{\rho}}\left(\mathbb{R}^{2}\right)=\left\{f \in \mathbb{L}_{2}\left(\mathbb{R}^{2}\right) \mid \operatorname{supp}(\mathscr{F}[f]) \subset B_{0, \boldsymbol{\rho}}\right\}, \\
\boldsymbol{\rho}>0 .
\end{gathered}
$$

The reason for this assumption is the well-known Nyquist theorem, which states that every band-limited function is determined by its values on a discrete grid. For example, if $u_{B}: \mathbb{R} \longrightarrow \mathbb{C}$ is bandlimited on a square, $\operatorname{supp}\left(\mathscr{F}_{F}\left[u_{B}\right]\right) \subset[-l / 2, l / 2] \times[-l / 2, l / 2]$, then

$$
\begin{gathered}
u_{B}(x, y)=\sum_{\left(k_{1}, k_{2}\right) \in \mathbb{Z}^{2}} u_{B}\left(\frac{2 \pi k_{1}}{l}, \frac{2 \pi k_{2}}{l}\right) \\
\times \operatorname{sinc}\left(\frac{l x}{2}-k_{1} \pi\right) \operatorname{sinc}\left(\frac{l y}{2}-k_{2} \pi\right),
\end{gathered}
$$

where the cutoff frequency $\boldsymbol{\rho}=l / 2$ is called the Nyquist frequency.

Actually, the Nyquist Theorem is another direct consequence of Theorem 4.3: take $H=\mathbb{L}_{2}([-\boldsymbol{\rho}, \boldsymbol{\rho}] \times$ $[-\boldsymbol{\rho}, \boldsymbol{\rho}])$, take $\mathbb{\|}=\mathbb{R}^{2}$, and take $\psi_{\mathbf{x}}=\frac{1}{2 \pi} e^{-i \boldsymbol{\omega} \cdot \mathbf{x}}, x=$ $(x, y) \in \llbracket$; then, $\mathscr{W}_{\psi}$ is the inverse Fourier transform

${ }^{10}$ In image analysis, orientation scores are sometimes called orientation bundles, but that name seems rather inappropriate. It is rather the domain of an orientation score, which is the Euclidean motion group, that can be considered as a principal fiber bundle over structure group $\mathbb{T}$, with respect to the left or right action of $\mathbb{T}$ on $G$. In these fibrations, the fibers are right cosets and left cosets, which are, respectively, the spirals $[g]=\left[\left(\mathbf{b}, e^{i \theta}\right)\right]=\left\{\left(R_{\theta} \mathbf{b}\right.\right.$, $\left.\left.e^{i\left(\theta+\theta^{\prime}\right)}\right) \mid e^{i \theta^{\prime}} \in \mathbb{T}\right\}$ and the straight lines $[g]=\left\{\left(\mathbf{b}, e^{i \theta-\theta^{\prime}}\right) \mid e^{i \theta^{\prime}} \in\right.$ $\mathbb{T}\}$.

${ }^{11}$ For example, in the discrete framework, the trivial case $\psi=\delta$ gives $\mathcal{W}_{\psi}[f]\left(\cdot, e^{i \theta_{k}}\right)=f$ for $k=1, \ldots, N$ with preservation of norm and thereby condition number 1 .

${ }^{12}$ Notice that the left-regular action of $G$ onto $\mathbb{L}_{2}^{\rho}\left(\mathbb{R}^{2}\right)$ is welldefined: if $f \in \mathbb{L}_{2}^{\boldsymbol{\rho}}\left(\mathbb{R}^{2}\right)$, then $u_{g} f \in \mathbb{L}_{2}^{\boldsymbol{\rho}}\left(\mathbb{R}^{2}\right)$ for all $g \in G$. 
restricted to $\mathbb{L}_{2}([-\boldsymbol{\rho}, \boldsymbol{\rho}] \times[-\boldsymbol{\rho}, \boldsymbol{\rho}])$ and thereby its range is the space $\left\{f \in \mathbb{L}_{2} \mid \operatorname{supp} \mathscr{F}[f] \subset[-\boldsymbol{\rho}, \boldsymbol{\rho}] \times[-\boldsymbol{\rho}, \boldsymbol{\rho}]\right\}$, which is the subspace of $\mathbb{L}_{2}\left(\mathbb{R}^{2}\right)$ consisting of bandlimited images, which is indeed a reproducing kernel Hilbert space with reproducing $\operatorname{kernel}\left(\psi_{\mathbf{x}}, \psi_{\mathbf{x}^{\prime}}\right)_{\mathbb{L}_{2}([-\boldsymbol{\rho}, \boldsymbol{\rho}] \times[-\boldsymbol{\rho}, \boldsymbol{\rho}])}=$ $\frac{1}{4 \boldsymbol{\rho}^{2} \pi^{2}} \operatorname{sinc}\left(\boldsymbol{\rho}\left(x-x^{\prime}\right)\right) \operatorname{sinc}\left(\boldsymbol{\rho}\left(y-y^{\prime}\right)\right)$. By Theorem 4.3, operator $\mathcal{W}_{\psi}$ is unitary, so it maps the orthonormal base $\left\{\mathbf{x} \mapsto e^{\frac{i m \pi x}{\boldsymbol{\rho}}} e^{\frac{i n \pi y}{\boldsymbol{\rho}}}\right\}_{m, n \in \mathbb{Z}}$ to the orthonormal base $\{\operatorname{sinc}(\boldsymbol{\rho} x-m \pi) \operatorname{sinc}(\boldsymbol{\rho} y-n \pi)\}$ in the functional Hilbert space $\left\{f \in \mathbb{L}_{2} \mid \operatorname{supp}(\mathscr{F}[f]) \subset[-\boldsymbol{\rho}, \boldsymbol{\rho}] \times[-\boldsymbol{\rho}, \boldsymbol{\rho}]\right\}$, and the result follows.

By (5.34), we consider images which are bandlimited on a disk rather than on a square, but this is just a subtlety as the reproducing kernel $\frac{1}{4 \rho^{2} \pi^{2}} \operatorname{sinc}(\rho(x-$ $\left.\left.x^{\prime}\right)\right) \operatorname{sinc}\left(\boldsymbol{\rho}\left(y-y^{\prime}\right)\right)=\mathscr{F}^{-1}\left[\boldsymbol{\omega} \mapsto e^{i \boldsymbol{\omega} \cdot\left(\mathbf{x}-\mathbf{x}^{\prime}\right)} 1_{[-\boldsymbol{\rho}, \boldsymbol{\rho}] \times[-\boldsymbol{\rho}, \boldsymbol{\rho}]}\right]$ is replaced by the kernel

$$
\begin{gathered}
J_{1}\left(\frac{\sqrt{\left(x-x^{\prime}\right)^{2}+\left(y-y^{\prime}\right)^{2}}}{\mathbf{\rho}}\right) \frac{\mathbf{\rho}}{\sqrt{\left(x-x^{\prime}\right)^{2}+\left(y-y^{\prime}\right)^{2}}} \\
=\mathscr{F}^{-1}\left[1_{B_{0, \boldsymbol{p}}}\right]\left(\mathbf{x}-\mathbf{x}^{\prime}\right) .
\end{gathered}
$$

Finally, we notice that from the practical point of view it does not make sense to store frequencies of order greater than $>l / 2$ if the signal contains $l$ samples, which is captured by the fact that a discrete Fourier transform of a discrete image $f:\{1, \ldots, n\} \times$ $\{1, \ldots, n\} \rightarrow \mathbb{C}$ is again of the form $\mathscr{F} f:\{1, \ldots, n\} \times$ $\{1, \ldots, n\} \rightarrow \mathbb{C}$. So a finite sample width puts an upper bound on the domain of the Fourier transform.

Definition 5.2. Let $\psi$ be an admissible wavelet. Then the wavelet transform $\mathcal{W}_{\psi}^{\boldsymbol{\rho}}: \mathbb{L}_{2}^{\boldsymbol{\rho}}\left(\mathbb{R}^{2}\right) \longrightarrow \mathbb{L}_{2}(G)$ is given by

$$
\mathcal{W}_{\psi}^{\rho}[f](g)=\left(\mathcal{U}_{g} \psi, f\right)_{\mathbb{L}_{2}\left(\mathbb{R}^{2}\right)}, \quad f \in \mathbb{L}_{2}^{\rho}\left(\mathbb{R}^{2}\right),
$$

for almost every $g \in G$.

Theorem 5.5. Let $\psi$ be an admissible wavelet, with $M_{\psi}(\boldsymbol{\omega})>0$ for all $\boldsymbol{\omega} \in \mathbb{R}^{2}$. Then the condition number cond $\left(\mathcal{W}_{\psi}^{\boldsymbol{\rho}}\right)$ of the wavelet transformation $\mathcal{W}_{\psi}^{\boldsymbol{\rho}}$ : $\mathbb{L}_{2}^{\mathbf{\rho}}\left(\mathbb{R}^{2}\right) \longrightarrow \mathbb{L}_{2}(G)$ is defined by

$$
\begin{gathered}
\operatorname{cond}\left(\mathcal{W}_{\psi}^{\mathbf{\rho}}\right)=\left\|\left(\mathcal{W}_{\psi}^{\mathbf{\rho}}\right)^{-1}\right\|\left\|W_{\psi}^{\boldsymbol{\rho}}\right\| \\
=\left(\sup _{f \in \mathbb{L}_{2}^{\mathbf{\rho}\left(\mathbb{R}^{2}\right)}} \frac{\|f\|_{\mathbb{L}_{2}\left(\mathbb{R}^{2}\right)}}{\left\|U_{f}\right\|_{\mathbb{L}_{2}(G)}}\right)\left(\sup _{f \in \mathbb{L}_{2}^{\mathbf{\rho}}\left(\mathbb{R}^{2}\right)} \frac{\left\|U_{f}\right\|_{\mathbb{L}_{2}(G)}}{\|f\|_{\mathbb{L}_{2}\left(\mathbb{R}^{2}\right)}}\right)
\end{gathered}
$$

and satisfies

$$
1 \leq\left(\operatorname{cond}\left(\mathcal{W}_{\psi}^{\boldsymbol{\rho}}\right)\right)^{2} \leq\left(\sup _{\|\boldsymbol{\omega}\| \leq \boldsymbol{\rho}} M_{\psi}^{-1}(\boldsymbol{\omega})\right)\left(\sup _{\|\boldsymbol{\omega}\| \leq \boldsymbol{\rho}} M_{\psi}(\boldsymbol{\omega})\right) .
$$

Proof. The proof is straightforward, for details see [12] p. 16.

Corollary 3. The stability of the (inverse) wavelet transformation $\mathcal{W}_{\psi}^{\boldsymbol{\rho}}: \mathbb{L}_{2}^{\boldsymbol{\rho}}\left(\mathbb{R}^{2}\right) \longrightarrow \mathbb{L}_{2}(G)$ is optimal if $M_{\psi}^{\boldsymbol{\rho}}(\boldsymbol{\omega})=$ constant for all $\boldsymbol{\omega} \in \mathbb{R}$, with $\|\boldsymbol{\omega}\| \leq \boldsymbol{\rho}$.

So in general, the more closely the function $M_{\psi}$ approximates the constant function, say 1 , on $B_{0, \boldsymbol{\rho}}$, the better the norm on $\mathbb{C}_{K}^{G}$ approximates the $\mathbb{L}_{2}(G)$-norm, and the better the stability of reconstruction. In case of a good approximation, one may use the following approximate reconstruction of the image:

$$
\begin{aligned}
& \tilde{\psi} * f=\mathscr{F}^{-1} {\left[\boldsymbol{\omega} \mapsto \frac{1}{2 \pi} \int_{0}^{2 \pi} \mathscr{F}_{f}\left[U_{f}\left(\cdot, e^{i \theta}\right)\right](\boldsymbol{\omega})\right.} \\
&\left.\times \mathscr{F}\left[\mathscr{R}_{e^{i \theta}} \psi\right](\boldsymbol{\omega}) d \theta\right],
\end{aligned}
$$

with the numerical benefit that in contrast to the true reconstruction (4.31), it does not use divisions in the Fourier domain.

In the theoretic case where the pixel size of our image $f$ converges to 0 and thereby the Nyquist frequency $\boldsymbol{\rho} \longrightarrow \infty$, the upper bound for stability tends to infinity. Since by $\psi \in \mathbb{L}_{1}\left(\mathbb{R}^{2}\right) \cap \mathbb{L}_{2}\left(\mathbb{R}^{2}\right) \Rightarrow M_{\psi} \in \mathbb{L}_{1}\left(\mathbb{R}^{2}\right)$, the continuous function $M_{\psi}^{-1}$ is unbounded on the whole $\mathbb{R}^{2}$. Relaxing the demand ${ }^{13}$ that $\psi \in \mathbb{L}_{2}\left(\mathbb{R}^{2}\right)$, by constructing a Gelfand Triple $\mathbb{H}^{2 k}\left(\mathbb{R}^{2}\right) \subseteq \mathbb{L}_{2}\left(\mathbb{R}^{2}\right) \subseteq$ $\mathbb{H}^{-2 k}\left(\mathbb{R}^{2}\right), k \in \mathbb{N}$ (for details see [11]), leads to an isometric wavelet transform from $\mathbb{L}_{2}\left(\mathbb{R}^{2}\right)$ to $\mathbb{L}_{2}(G)$, with the condition number equal to 1 .

\section{CLASSES OF WAVELETS THAT ALLOW WELL-POSED RECONSTRUCTION}

By Corollary 3, wavelets $\psi$ with $M_{\psi}=1_{B_{0, p}}$ induce optimal stability of the (inverse) wavelet transform. Because of the discontinuity at $\rho=\|\boldsymbol{\omega}\|=\boldsymbol{\rho}$, in practice this choice causes numerical problems with the discrete inverse Fourier transform.

To avoid this practical problem, we mainly focus on wavelets $\psi$, with either $M_{\psi}(\rho)=\mathscr{G}_{\sigma}(\|\omega\|)$, a Gaussian kernel with standard deviation $\sigma \approx \rho / 2$, or $M_{\psi}(\rho)=$ $M_{N}\left(\sigma^{2} \rho^{2}\right), N \in \mathbb{N}, \sigma>0, \rho=\|\omega\|$, where

$$
M_{N}\left(\rho^{2}\right)=e^{-\rho^{2}} \sum_{k=0}^{N} \frac{\rho^{2 k}}{k !} \leq 1 .
$$

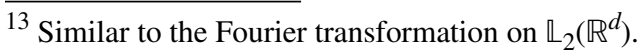




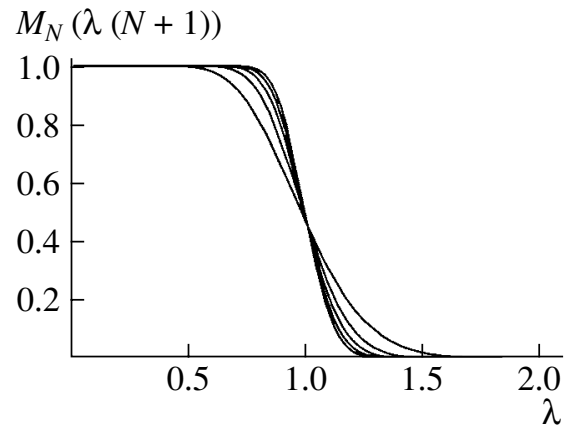

Fig. 4. Plots of $\lambda \mapsto \mu_{N}\left(\rho^{2}\right)$, with $\rho^{2}=\lambda(N+1)$, for $N=$ $20,40,60,80,100$.

In both cases, the function $M_{\psi}$ smoothly approximates $1_{B_{0, \mathrm{p}}}$, and thereby guarantees a stable reconstruction. In what follows we will call a wavelet $\psi \in$ $\mathbb{L}_{2}\left(\mathbb{R}^{2}\right) \cap \mathbb{L}_{1}\left(\mathbb{R}^{2}\right)$, with such a $M_{\psi}$, a proper wavelet.

For more analysis on $M_{N}$ given by (6.37), we refer to [12] p. 18, where it is shown that the condition number of wavelets $\psi$ with $M_{\psi}(\rho)=M_{N}\left(\frac{\rho N}{\rho}\right)$ satisfies $\left(\operatorname{cond}\left(\mathcal{W}_{\psi}^{\boldsymbol{\rho}}\right)\right)^{2} \leq 1 \cdot \mathcal{M}_{N}^{-1}(N+1)=2+O\left(\frac{1}{\sqrt{N}}\right)$, which is indeed close to optimal stability, which coincides with Fig. 4.

\subsection{A Simple Approach to Parameterization of Proper Wavelet Classes}

The following lemma shows us a simple but practical approach to obtaining proper wavelets $\psi$ with the same $M_{\psi}(\boldsymbol{\omega})=M(\rho), \rho=\|\boldsymbol{\omega}\|$, where $\mathcal{M}$ ensures a stable reconstruction.

Lemma 6.3. Let $A: S^{1} \longrightarrow \mathbb{C} \backslash \mathbb{R}^{-}$be such that

$$
\frac{1}{2 \pi} \int_{0}^{2 \pi}|A(\varphi)| d \varphi=1
$$

then the wavelet $\psi=\mathscr{F}^{-1}[\boldsymbol{\omega} \mapsto \sqrt{A(\varphi) \mathcal{M}(\rho)}]$ has $M_{\psi}(\boldsymbol{\omega})=M(\rho)$ for all $\boldsymbol{\omega} \in \mathbb{R}^{2}$.

Proof.

$$
M_{\psi}(\boldsymbol{\omega})=\frac{1}{2 \pi} \int_{0}^{2 \pi}\left|\mathscr{F}\left(\mathscr{R}_{\theta} \psi\right)(\boldsymbol{\omega})\right|^{2} d \theta=
$$

$\frac{1}{2 \pi} \int_{0}^{2 \pi}|\sqrt{A(\phi-\theta) M(\rho)}|^{2} d \theta=M(\rho)$, for all $\omega \in \mathbb{R}^{2}$, $\|\omega\|=\rho$.

Lemma 6.3 is easily translated to the discrete framework $\mathbb{R}^{2} \times \mathbb{T}_{N}$; see (4.32), where condition (6.38) must be replaced by

$$
\frac{1}{N} \sum_{k=0}^{N}|A(\phi-k \Delta)|=1
$$

If, moreover, $\frac{1}{2 \pi} \int_{0}^{2 \pi} \sqrt{A(\phi)} d \phi \approx 1$, we have a fast/simple approximate reconstruction:

$$
\begin{gathered}
\tilde{f}(\mathbf{x})=\frac{1}{2 \pi} \int_{0}^{2 \pi} U_{f}(\mathbf{x}, \theta) d \theta \\
\approx \mathscr{F}^{-1}\left[\boldsymbol{\omega} \mapsto \sqrt{M(\rho)} \mathscr{F}_{F}[f](\boldsymbol{\omega})\right](\mathbf{x}) \\
=\left(\sqrt{M_{\psi}} * f\right)(\mathbf{x}), \text { for almost every } \mathbf{x} \in \mathbb{R}^{2} . \\
M_{\psi} \approx 1_{B_{0, \mathbf{p}}} \Rightarrow \tilde{f} \approx f \in \mathbb{L}_{2}^{\mathbf{\rho}}\left(\mathbb{R}^{2}\right),
\end{gathered}
$$

which coincides with the exact reconstruction in the simple example of Section 3 (see Fig. 6).

On the one hand, the class of proper wavelets induced by Lemma 6.3 is fairly wide, which allows us to select a proper wavelet that is a good detector of elongated structures (like blood vessels or catheters in medical images). On the other hand, this class of wavelets seems rather restrictive, as the Fourier transform of the wavelet, $\mathscr{F}[\psi]$, is assumed to be polar-separable, with a (more or less) fixed radial component $R$. The

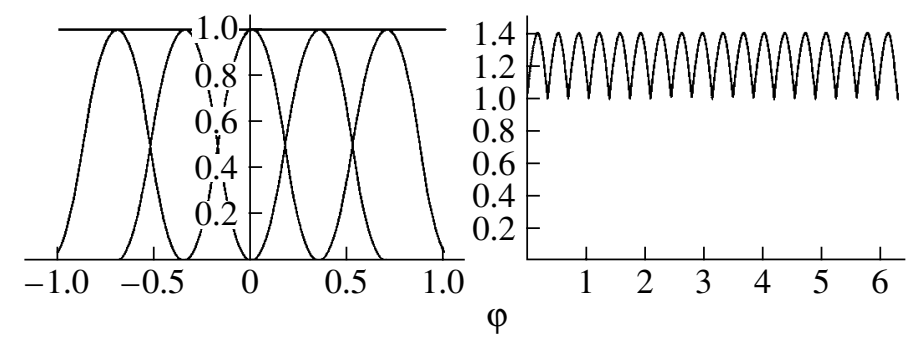

Fig. 5. Left: Plots of $A(\phi-k \Delta)$ (see (6.41)), with $\Delta=\frac{2 \pi}{18}, k=-2,-1,0,1,2$. Notice that it follows by the point-symmetries at $\left\{k+\left(\frac{\Delta}{2}\right), \frac{1}{2}\right\}$ that $\frac{1}{N} \sum_{k=0}^{N-1}|A(\phi-k \Delta)|=1$. Right: Graph of $\phi \mapsto \frac{1}{N} \sum_{k=0}^{N-1} \sqrt{A(\phi-k \Delta)} \approx 1$. 


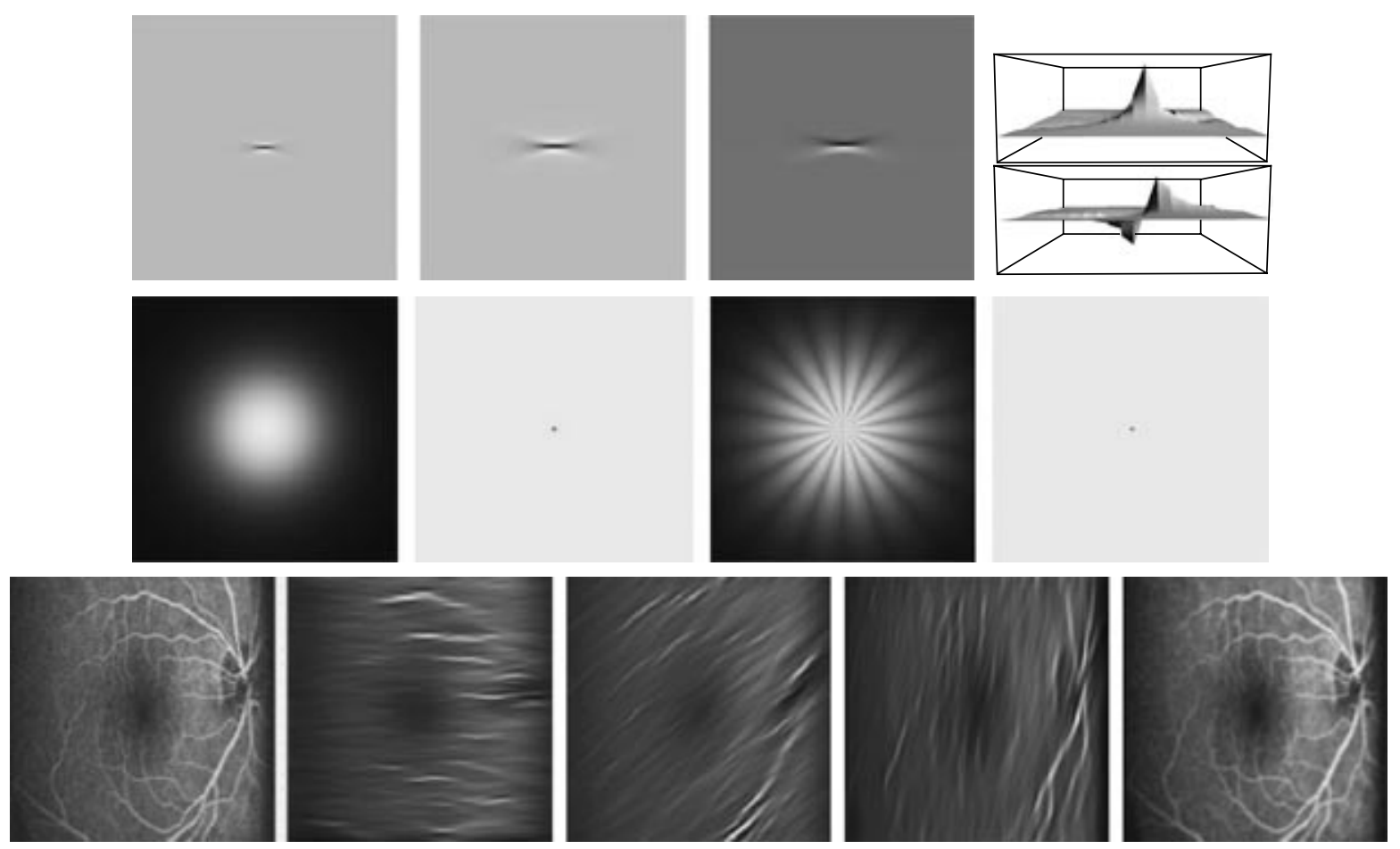

Fig. 6. Upper row: Plots of the graph of the real and imaginary part of $\psi=\mathscr{F}^{-1}[\omega \mapsto \sqrt{A(\varphi) M(\rho)}$, with $A(\varphi)$ given by (6.41) and $\mathcal{M}(\rho)=G_{s}(\rho), s=800$, determined by the discrete inverse Fourier transform of $\omega \mapsto \sqrt{A(\varphi) \mathcal{M}(\rho)}$ sampled on a $256 \times 256$ equidistant grid. From left to right: Density plots (grey-values have been mapped to full range and inverted for the sake of clarity) of $\mathfrak{R}(\psi)$ at true size, $\mathfrak{i}(\psi)$ and $\mathfrak{S}(\psi)$ with 2 times zoom, and finally two SD plots of the graphs of $\mathfrak{i}(\psi)$ and $\mathfrak{\Im}(\psi)$. Second row: From left to right: plots of $M_{\psi}$ and Fourier inverse $\tilde{\psi}=\mathscr{F}^{-1}\left[M_{\psi}\right]$, sum of all rotated kernels in Fourier and spatial domain. Third row: MRI-image of the retina, three slices $U_{f}^{N}\left(e^{i k \Delta}, \cdot\right), k=0,2,4$ of the discrete orientation score $U_{f}^{N}$ and fast approximative reconstruction, (6.40) which is close to the exact reconstruction.

separability restriction is not harmful in practice since $\mathbb{L}_{2}^{\mathbf{\rho}}\left(\mathbb{R}^{2}\right)=\mathbb{L}_{2}\left(S^{1}\right) \otimes \mathbb{L}_{2}((0, \boldsymbol{\rho}), \rho d \rho)$, so by choosing a basis in $\mathbb{L}_{2}\left(S^{1}\right)$ and $\mathbb{L}_{2}((0, \boldsymbol{\rho}), \rho d \rho)$, the Fourier transform $\mathscr{F}[\psi]$ of a line detector wavelet is always a superposition of separable wavelets.

The restriction of the radial component(s) is a more serious problem. Mostly, typical line detecting convolution kernels have a radial component with a much smaller width than $M(\rho)$. Therefore, replacement of the radial component $R$ by $\mathcal{M}(\rho)$ will affect the radial shape of the wavelet, but preserve the angular part of the wavelet.

Example 1. Consider $N=18$ discrete orientations, so that $\Delta=\frac{2 \pi}{N}$. The idea is to "fill a cake by pieces of cake" in the Fourier domain. In order to avoid high frequencies in the spatial domain, these pieces must be smooth, and therefore they must overlap.

Let $\psi=\mathscr{F}^{-1}\left[\boldsymbol{\omega} \mapsto \sqrt{A(\varphi) \mathcal{M}(\rho)}\right.$ and let $A: S^{1} \longrightarrow$ $\mathbb{R}^{+}$be given by

$$
A(\varphi)=\left\{\begin{array}{l}
-\left(\frac{2}{\Delta^{2}} \varphi^{2}\right)^{2} \varphi^{2}+1 \text { if }|\varphi| \leq \frac{\Delta}{2} \\
\left(\frac{2}{\Delta^{2}}\right) \varphi^{2}-\frac{4}{\Delta}|\varphi|+2 \text { if } \frac{\Delta}{2} \leq|\varphi| \leq \Delta, \\
0 \text { else } \\
\varphi \in[-\pi, \pi) ;
\end{array}\right.
$$

then it is easily checked that $M_{\psi}(\boldsymbol{\omega})=\mathcal{M}(\rho), \rho=\|\boldsymbol{\omega}\|$ (see (6.39) and Fig. 5). Now we have $\frac{1}{N} \sum_{k=0}^{N}|\sqrt{A(\phi-k \Delta)}| \approx 1$ (again see Fig. 5), which allows us to use the practical approximative reconstruction given by (6.40). Moreover, the real valued part of the wavelet is useful for line detection and the imaginary part of the kernel is appropriate for edge detection (see Fig. 6). For line enhancement (with comparison to the usual line detection) via orientation score, see Fig. 7. Notice that if $A_{1}: S^{1} \longrightarrow \mathbb{R}^{+}$and $A_{2}: S^{1} \longrightarrow \mathbb{R}^{+}, A_{i} \in$ $\mathbb{L}_{1}\left(S^{1}\right) \cap \mathbb{L}_{2}\left(S^{1}\right)$ satisfy condition $(6.38)$, then so does 

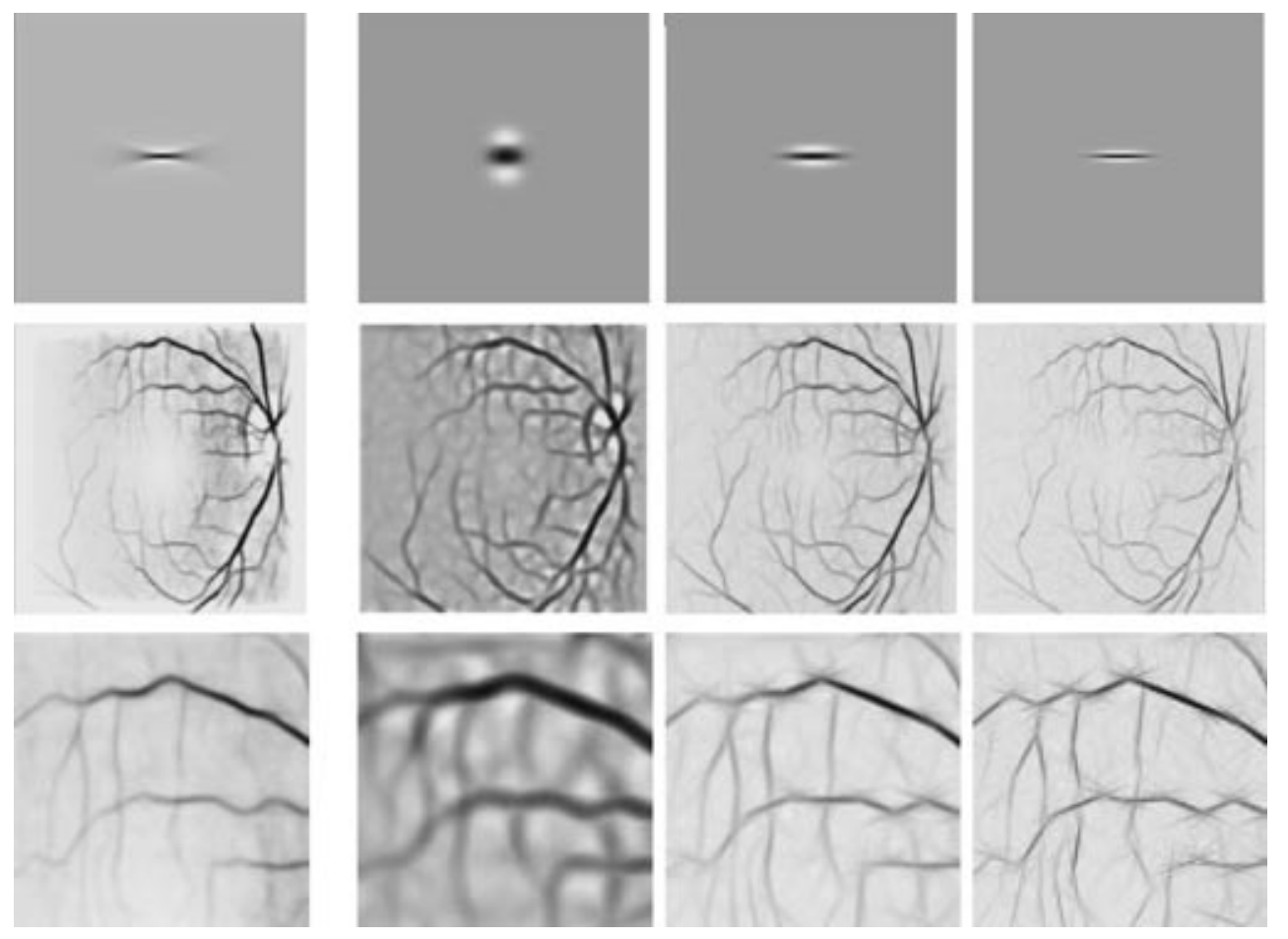

Fig. 7. Top row, from left to right, a proper wavelet $\psi=\mathscr{F}^{-1}[\omega \mapsto \sqrt{A(\varphi) \mu(\rho)}]$ as shown in the previous figure, second order derivatives of anisotropic Gaussian kernel $\gamma=1, \gamma=4, \gamma=7$ (see (6.43)). For the sake of illustration, we zoomed in by a factor of 2 in all cases. Middle row: detection of elongated structures via orientation scores constructed by wavelet, $\left.\mathscr{W}_{\psi}^{*}\left[\frac{\left|\mathfrak{R}\left(U_{f}\right)-\min \left\{\mathfrak{R}\left(U_{f}\right)\right\}\right|^{2}}{\max \left\{\left|\mathfrak{R}\left(U_{f}\right)-\min \left\{\mathfrak{R}\left(U_{f}\right)\right\}\right|\right.}\right\}\right]$ and comparison with the usual line detection in image analysis with second order Gaussian kernel, for $\gamma=1, \gamma=4$ and $\gamma=7$ (see (6.42)). Bottom row: enlarged small fragment of images. This figure shows us that there exist proper wavelets (with the advantage of a stable reconstruction) that can be used for line detection, which is at least as good as the usual approach. In fact, when a stable reconstruction is demanded, the tail of a proper wavelet typically spreads out more naturally. Therefore proper wavelets, which look locally at the center like the usual line detectors, deal better with elongated lines which are slightly curved, which is usually the case in practical applications such as catheter, guide wire, and blood vessel detection.

the periodic convolution $A_{1} * A_{2}: S^{1} \longrightarrow \mathbb{R}^{+}$given by $\left(A_{1} * A_{2}\right)(\phi)=\int_{0}^{2 \pi} A_{1}(\phi-\psi) A_{2}(\psi) d \psi$. This enables us to generate explicit examples, which are similar to the wavelet in Example 1 in the same sense of splitting a cake into equal and smooth overlapping pieces. For example, take $A_{i}(\phi)=\sum_{k=0}^{N} \chi_{k}(\phi), i=1,2$, where $\chi_{x}(\phi)=$ $\left(\tau_{k \Delta} \chi_{0}\right)(\phi)=\chi_{0}(\phi-k \Delta)$ and $\chi(\phi)=1_{-\Delta / 2, \Delta / 2}(\phi)$; then by $\chi_{i} * \chi_{j}=\tau_{(i+j) \Delta}\left(\chi_{0} * \chi_{0}\right)$, we obtain a first order B-spine representation of $1_{S^{1}}$. If we repeat this process $n$ times, we obtain a $n+1$-th order $B$-spline representation of $1_{S^{1}}(\phi)=\sum_{k=0}^{N-1}\left(\chi_{0} *^{(n)} \chi_{0}\right)(\phi-k \Delta)$

Example 2. The most common method of line detection in image analysis is by means of the largest eigenvalue $\lambda_{1}(\mathbf{b})$ of the Hessian matrix $H u(\mathbf{b})$ of second order Gaussian derivatives

$$
H u(\mathbf{b})=\left(\begin{array}{ll}
u_{x x}(\mathbf{b}) & u_{x y}(\mathbf{b}) \\
u_{x y}(\mathbf{b}) & u_{y y}(\mathbf{b})
\end{array}\right)
$$

where $u(\mathbf{b}, s)=\left(G_{s} * f\right)(\mathbf{b})$, and $\mathbf{s}=\frac{1}{2} \sigma^{2}>0$ is the socalled scale space representation of image $f \in \mathbb{L}_{2}\left(\mathbb{R}^{2}\right)$. We notice that

$$
\begin{gathered}
\lambda_{1}(\mathbf{b})=\max _{\theta \in[0,2 \pi)}\left|\left(R_{\theta} \mathbf{e}_{x}\right)^{T} H u(\mathbf{b})\left(R_{\theta} \mathbf{e}_{x}\right)\right| \\
=\max _{\theta \in[0,2 \pi)}\left\{\left|u_{\xi \xi}\right| \xi=(\cos \theta) x+(\sin \theta) y\right\} \\
=\max _{\theta \in[0,2 \pi)}\left|\left(f * \mathscr{R}_{\theta}\left(G_{s}\right)^{(2,0)}\right)(\mathbf{b})\right| \\
=\max _{\theta \in[0,2 \pi)}\left|W_{G_{s}^{(2,0)}}[f]\left(\mathbf{b}, e^{i \theta}\right)\right|=\max _{\theta \in[0,2 \pi)}\left|U_{f}\left(\mathbf{b}, e^{i \theta}\right)\right|,
\end{gathered}
$$

so the usual method in image analysis is in fact a maximum intensity projection of an orientation score with a second order derivative of the Gaussian kernel. Such a second order derivative of a Gaussian clearly distin- 
guishes lines from edges and it can easily be stretched by replacing the Gaussian kernel by an anisotropic Gaussian kernel:

$$
\begin{gathered}
G_{s, \gamma}^{(2,0)}(x, y)=G_{s / \gamma}^{(2)}(x) G_{s}(y) \\
=\frac{\gamma}{16 \pi s^{3}}\left(\gamma x^{2}-2 s\right) e^{-\frac{\gamma x^{2}}{4 s}} e^{-\frac{y^{2}}{4 s}}, \quad s>0, \gamma>0 .
\end{gathered}
$$

Its Fourier transform is given by

$$
\begin{gathered}
\mathscr{F}\left[G_{s, \gamma}^{(2,0)}\right](\boldsymbol{\omega})=-\rho^{2}(\cos \varphi)^{2} e^{-\frac{1}{2}\left(\frac{\sigma \rho}{\kappa}\right)^{2}} e^{-\frac{1}{2}(\rho \sigma \sin \varphi)^{2}\left(1-\frac{1}{\kappa^{2}}\right)} \\
\boldsymbol{\omega}=(\rho \cos \varphi, \rho \sin \varphi),
\end{gathered}
$$

from which we immediately deduce that $\mathrm{M}_{\psi}$, for $\psi=$ $G_{s, \gamma}^{(2,0)}$ is not at all desirable; a well-posed reconstruction is not possible. Therefore, we will construct new wavelets that are locally similar to $\psi=G_{s, \gamma}^{(2,0)}$ but that do allow a well-posed reconstruction. In [12] p. 23, we decomposed $\psi=G_{s, \gamma}^{(2,0)}$ into steerable components, which is less trivial than it seems for $\gamma \neq 1$. Instead of using $G_{s, \gamma}^{(2,0)}$ as a line detector wavelet, we propose the wavelets $\psi_{\gamma, \beta}$, whose Fourier transforms are given by

$$
\begin{aligned}
& \mathscr{F}_{F}\left[\psi_{\gamma, \beta}\right](\boldsymbol{\omega})=\cos ^{2} \varphi e^{-\frac{1}{2} \frac{(\rho \sigma)^{2}}{\gamma^{2}}} e^{-\frac{\beta}{2} \sin ^{2}(\phi) \sigma^{2}\left(1-\frac{1}{\gamma^{2}}\right)}, \\
& \beta \in\left[0, \frac{1}{2}\right),
\end{aligned}
$$

which is locally similar to $G_{s, \gamma}^{(2,0)}$, with the same angular components, but which has the following practical advantages over $G_{s, \gamma}^{(2,0)}$ :

(1) It allows a well-posed approximate reconstruction.

(2) It is a simple steerable filter, in the sense that its expansion in $\{\cos m \varphi\}$ (in the Fourier domain) is simple and can be truncated at low $m,(m=16)$.

(3) Its shape is more natural for line detection, since the objective elongated structures may have some curvature.

See Fig. 6.5 in our technical report [12]. In the next subsection, we consider a more general, but also more difficult approach to obtaining (parameterizations of) proper wavelets.

\subsection{A General Approach to Parameterization of Proper Wavelet Classes}

We will derive $\psi$ and $M_{\psi}$ explicitly in a Fourier invariant orthonormal base. Some (classes of) proper wavelets pop up in a very natural way and they are essentially different from the ones in the previous section.
Notice that $\mathbb{L}_{2}\left(\mathbb{R}^{2}\right)=\mathbb{L}_{2}\left(S^{1}\right) \otimes \mathbb{L}_{2}((0, \infty), r d r)$. Decompose the angular part $\mathbb{L}_{2}\left(S^{1}\right)$ into irreducible representations $^{14}$ :

$$
\mathbb{L}_{2}\left(S_{1}\right)=\bigoplus_{m \in \mathbb{Z}} \mathscr{D}^{(m)}=\bigoplus_{m \in \mathbb{Z}}\left\langle Y_{m}\right\rangle=\bigoplus_{m \in \mathbb{Z}}\left\langle\phi \mapsto \frac{e^{-i m \phi}}{\sqrt{2 \pi}}\right\rangle .
$$

Let $\left\{\gamma_{m n}\right\}_{n \in N}$ in $\mathbb{L}_{2}((0, \infty), r d r)$ be an orthonormal base for each $m \in \mathbb{Z}$; then $\left(\gamma_{m n} \otimes Y_{m}\right)(r, \phi)=\gamma_{m l}(r) Y_{m}(\phi)$ is an orthonormal base in $\mathbb{L}_{2}\left(\mathbb{R}^{2} ; r d r\right)$. As a result, we can expand any wavelet $\psi \in \mathbb{L}_{2}\left(\mathbb{R}^{d}\right)$ in terms of this basis:

$$
\begin{aligned}
\psi(r, \phi) & =\sum_{n \in \mathbb{Z} n=0} \sum_{m n}^{\infty} \gamma_{m n}(r) Y_{m}(\phi)\left(\gamma_{m n} \otimes Y_{m}, \psi\right)_{\mathbb{L}_{2}\left(\mathbb{R}^{2}\right)} \\
& =\sum_{m \in \mathbb{Z}} \psi_{m}(r, \phi)=\sum_{m \in \mathbb{Z}} g_{m}(r) \psi_{m}(\phi) .
\end{aligned}
$$

By the Bochner-Hecke Theorem (see Appendix A), the Fourier Transform $\mathscr{F}[\psi]$ of the wavelet $\psi \in \mathbb{L}_{2}\left(\mathbb{R}^{d}\right)$ is given by

$$
\mathscr{F}[\psi](\rho, \bar{\phi})=\sum_{m \in \mathbb{Z}} \tilde{g}_{m}(\rho) Y_{m}(\phi),
$$

with $\quad \tilde{g}_{m} \in \mathbb{L}_{2}\left(\mathbb{R}^{2} ; \quad r d r\right)$ given by $\tilde{g}_{m}(\rho)=$ $\rho^{1 / 2} \mathcal{H}_{m}\left[r^{1 / 2} g_{m}(r)\right](\rho)$, where the unitary Hankel Transform $\mathscr{H}_{m}: \mathbb{L}_{2}((0, \infty)) \longrightarrow \mathbb{L}_{2}((0, \infty))$ is given by (A.83). Given such a wavelet $\psi \in \mathbb{L}_{2}\left(\mathbb{R}^{d}\right)$, the orientation score $U_{f}$ can be written

$$
\begin{aligned}
U_{f}\left(\mathbf{b}, e^{i \theta}\right) & =\left(\overline{\mathscr{R}_{R_{\theta}} \check{\psi}} * f\right)(\mathbf{b})=\left(\overline{\mathscr{R}_{R_{\theta+\pi}} \psi} * f\right)(\mathbf{b}) \\
& =\sum_{m \in \mathbb{Z}}(-1)^{m} \frac{e^{-i m \theta}}{\sqrt{2 \pi}}\left(\overline{\psi_{m}} * f\right)(\mathbf{b}) .
\end{aligned}
$$

If the radial functions do not depend strongly on $m$, the wavelet will be directed along $\phi=0$ (and thereby the rotated kernel will be directed along $\phi=\theta$ ). The real part of the orientation score (constructed by a real valued wavelet which is even around $\phi=0$ ) reveals elongated line structures, whereas the imaginary part (constructed by a real valued wavelet which is odd around $\phi=0$ ) reveals elongated edge structures.

The function $M_{\psi}: \mathbb{R}^{d} \longrightarrow \mathbb{R}$, which by Theorem 5.5 completely determines the well-posedness of $f \longleftrightarrow U_{f}$, is now given by

$$
\begin{gathered}
M_{\psi}(\boldsymbol{\omega})=\int_{S O(d)}\left|\mathscr{F}_{(}\left(\mathscr{R}_{R} \psi\right)(\boldsymbol{\omega})\right|^{2} d R=\sum_{m \in \mathbb{Z}}\left|\tilde{g}_{m}(\rho)\right|^{2}, \\
\text { with } \rho=\|\boldsymbol{\omega}\| .
\end{gathered}
$$

\footnotetext{
14 The irreducible representations of commutative groups are always defined on one dimensional subspace.
} 
6.2.1. Expansion of $\psi$ and $M_{\psi}$ in a Fourier Invariant polar base. Although Eq. (6.47) is of rather simple form, it still includes the operator $\rho^{-1 / 2} \mathcal{H}_{m} r^{1 / 2}$. This unitary operator on $\mathbb{L}_{2}((0, \infty) ; r d r)$ has the following complete set of orthonormal eigenfunctions $h_{n}^{m}$ (for details see Appendix A):

$$
\begin{gathered}
h_{n}^{m}(r)=\left(\frac{2 n !}{(m+n) !}\right)^{1 / 2} r^{m} e^{-r^{2} / 2} / L_{n}^{(m)}\left(r^{2}\right), \\
r>0, \quad m \geq 0 .
\end{gathered}
$$

For $m<0$, we define $h_{n}^{m}=h_{n}^{|m|}$. Therefore, we expand $\psi$ and express $M_{\psi}$ in this angular-irreducible radially Fourier invariant basis, which are also the eigen functions of the 2D-Harmonic oscillator ${ }^{15}$ :

$$
\begin{gathered}
\psi(\mathbf{x})=\sum_{m \in \mathbb{Z} l=0} \sum_{m}^{\infty} \alpha_{m}^{n}\left(Y_{m} \otimes h_{n}^{m}\right)(\phi, r), \\
\mathscr{F} \psi(\boldsymbol{\omega})=\sum_{m \in \mathbb{Z} l=0} \sum_{i=1}^{\infty}(i)^{|m|}(-1)^{n+m} \alpha_{m}^{n}\left(Y_{m} \otimes h_{n}^{m}\right)(\varphi, \rho), \\
\left(\mathscr{R}_{R_{\theta}} \psi\right)(\mathbf{x})=\sum_{m \in \mathbb{Z} n=0} \sum_{m}^{\infty} \alpha_{m}^{n} e^{+i m \theta}\left(Y_{m} \otimes h_{n}^{m}\right)(\phi, r),(6.49) \\
\left.M_{\psi}(\boldsymbol{\omega})=\sum_{m=-\infty}^{\infty} \mid \sum_{n=0}^{\infty}(-1)^{n} \alpha_{m}^{n} h_{n}^{m}(\rho)\right)\left.\right|^{2} \\
=\sum_{m=-\infty}^{\infty} \sum_{n=0}^{\infty} \sum_{n^{\prime}=0}^{\infty}(-1)^{n+m^{\prime}} \alpha_{m}^{n} \overline{\alpha_{m}^{n}} h_{n}^{m}(\rho) h_{n^{\prime}}^{m}(\rho) .
\end{gathered}
$$

In the rest of this section, we will construct wavelets $\psi_{N_{1}, N_{2}}$, with coefficients $\alpha_{m}^{n}=0$, for $|m|>N_{1}$ and $n>$ $N_{2}$, for fixed $N_{1}, N_{2} \in \mathbb{N}$, i.e.,

$$
\psi_{N_{1}, N_{2}}=\sum_{m=-N_{1}}^{N_{1}} \sum_{n=0}^{N_{2}} \alpha_{m}^{n} Y_{m} \otimes h_{n}^{m}
$$

such that

$$
M_{\Psi_{N}}=M_{N}, \text { with } N=N_{1}+2 N_{2},
$$

where we recall that $\mu_{N}$ is given by (6.37). We consider (6.50) as an equation in variable $\psi$, determined by its coefficients $\left\{\boldsymbol{\alpha}_{m}\right\}_{|m|=0}^{N_{1}}=\left\{\left\{\alpha_{m}^{n}\right\}_{n=0}^{N_{2}}\right\}_{|m|=0}^{N_{1}}$ and thereby

15 The self-adjoint operators $\mathscr{F}, \mathscr{R}_{R}$ and the harmonic oscillator $\Delta-$ $\|\mathbf{x}\|^{2}$ commute and have a common base of eigen functions which are indeed given by $Y_{m} \otimes h_{n}^{m}$. we obtain $M_{\psi_{N}}(\boldsymbol{\omega})=M_{N}(\rho)$ for almost every $\rho=\|\boldsymbol{\omega}\|>$ 0 , if and only if

$$
\begin{gathered}
\sum_{|m|=0}^{N_{1}} \sum_{n=0}^{N_{2}} \sum_{n^{\prime}=0}^{N_{2}}(-1)^{n+n^{\prime}} \sqrt{\frac{2 n !}{(n+|m|) !}} \sqrt{\frac{2 n^{\prime} !}{\left(n^{\prime}+|m|\right) !}} \\
\times \rho^{2|m|} L_{n}^{(m)}\left(\rho^{2}\right) L_{n^{\prime}}^{(m)}\left(\rho^{2}\right)=\sum_{q=0}^{N} \frac{\rho^{2 q}}{q !} \\
\sum_{k=0}^{2 N_{2}} \sum_{|m|=0}^{N_{1}} \boldsymbol{\alpha}_{m}^{\dagger} A_{k}^{m} \boldsymbol{\alpha}_{m} \rho^{2(k+|m|)}=\sum_{q=0}^{N} \frac{\rho^{2 q}}{q !},
\end{gathered}
$$

where the $\left(N_{2}+1\right) \times\left(N_{2}+1\right)$ positive symmetric matrices $A_{k}^{m}$ are given by $\left[A_{k}^{m}\right]_{n^{\prime}}^{n}=\sum_{j=0}^{k}\left(\mathbf{a}_{j}^{m}\right)_{n}\left(\mathbf{a}_{(k-j)}^{m}\right)_{n^{\prime}}$, with

$$
=\left\{\begin{array}{l}
\left.(-1)^{n} \sqrt{\frac{2(n !)}{(n+|m|) !}} d_{k}^{m, n}\right)_{n} \\
=(-1)^{n+k} \sqrt{\frac{2(n !)}{(n+|m|) !}} \frac{1}{k !}\left(\begin{array}{c}
n+|m| \\
n-k
\end{array}\right) \text { if } k \leq n, \\
0 \text { else, }
\end{array}\right.
$$

where $d_{k}^{m, n}=\frac{(-1)^{k}}{k !}\left(\begin{array}{c}n+|m| \\ n-k\end{array}\right)$ equals the coefficient of $\rho^{2 k}$ in $L_{n}^{(|m|)}\left(\rho^{2}\right)$, cf. [1].

Now in the left hand side of (6.50), we introduce the summation index $q=|m|+k$ and assume $\boldsymbol{\alpha}_{-m}=\boldsymbol{\alpha}_{m} \in$ $\mathbb{R}^{N_{2}+1}$ for all $m$, (i.e., the wavelet is symmetric around its direction); then we obtain

$$
\begin{gathered}
\sum_{q=0 k=\max \left\{0, q-N_{1}\right\}}^{N} \boldsymbol{\alpha}_{m}^{T} A_{k}^{q-k} \boldsymbol{\alpha}_{q-k} \rho^{2 q}=\sum_{q=0}^{N} \frac{\rho^{2 q}}{q !} \\
\Leftrightarrow \sum_{k=\max \left\{0, q-N_{1}\right\}}^{2 N_{2}} \boldsymbol{\alpha}_{m}^{T} A_{k}^{q-k} \boldsymbol{\alpha}_{q-k} \rho^{2 q}=\frac{1}{q !}, \\
\text { for } q=0, \ldots, N,
\end{gathered}
$$

which are $N+1=N_{1}+2 N_{2}+1$ equations for $\left(N_{1}+\right.$ 1) $\left(N_{2}+1\right)$ variables $\left\{\boldsymbol{\alpha}_{m}\right\}_{m=0}^{N_{1}}$. 

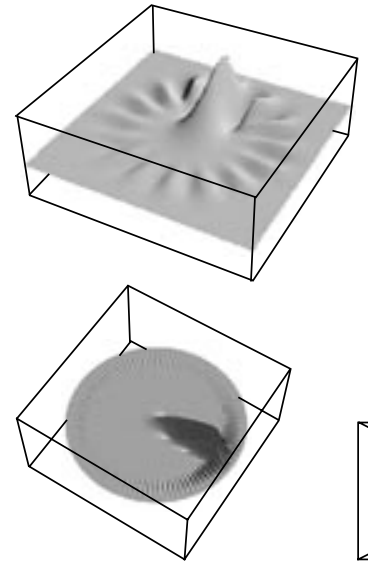
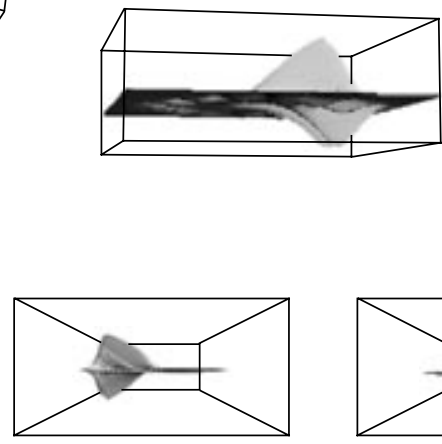
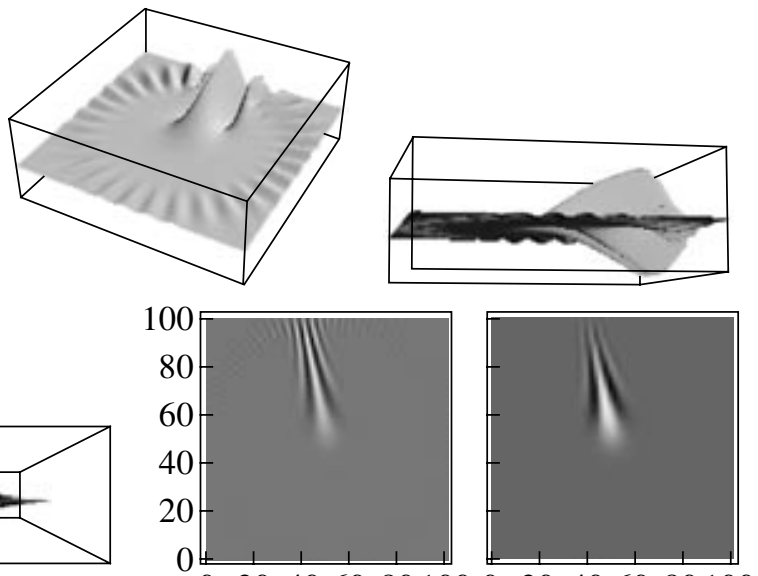

Fig. 8. Top row left: two different viewpoint plots of graphs $\psi_{N=15}^{0}$. Top row right: two different viewpoint plots of graphs $\psi_{N=30}^{0}$. Bottom row left: three plots of graph $\Re\left(\psi_{N=100}^{0}\right)$. Bottom row right: $100 \times 100$-pixel grey-value plots of $\Re\left(\psi_{N=100}^{0}\right)$ and Gaussian blurred (with $\sigma=0.8$ pixels). Notice that the kernel becomes sharper and the wiggle-ring vanishes as $N$ increases. The locally highly oscillatory behavior within $\Re\left(\psi_{N=100}^{0}\right)$, which may seem awkward, is not really harmful since it disappears immediately by convolution with Gaussian kernel with tiny scale (see also (6.54)).
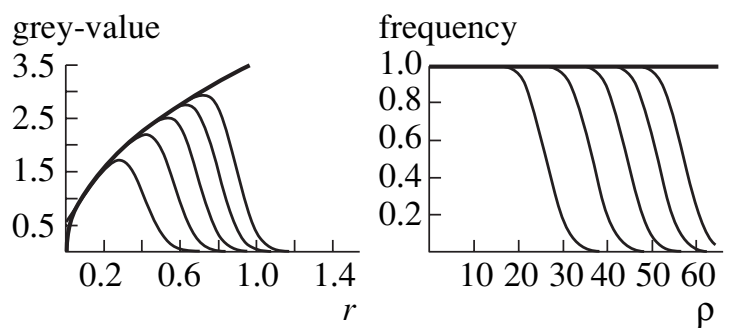

Direction-Variance

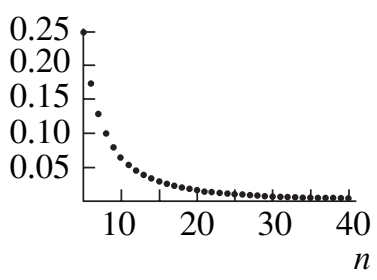

Fig. 9. Left: The graphs of the kernel $\psi_{N}^{0}(\mathbf{x})$ cut off at $N=10,20,30,40,50$ with $\sigma=1 / 8$, restricted to its main direction $\phi=0$. Notice that the peaks move out as $\mathrm{m}$ increases. Notice that the asymptotic formula derived for $\psi_{\infty}^{0}(r, \phi=0)=$ $(8 \pi)^{1 / 4} \sqrt{r}\left(1-\frac{1}{16 r^{2}}+O\left(\frac{1}{r^{4}}\right)\right)=(8 \pi)^{1 / 4} \sqrt{r}+O\left(r^{-\frac{3}{2}}\right)$ (see Appendix $\mathrm{C}$ in [12]) is a good approximation (we included the graph of $r \mapsto(8 \pi)^{1 / 4} \sqrt{r}$ ). Middle: The corresponding functions $\mathcal{M}_{\psi_{N}^{0}}=\mathcal{M}_{N}$, which indeed approximate 1 as $N \longrightarrow \infty$. Right: Angular variance of $\psi_{N}^{0}$ plotted as a function in $N$.

Example. The special case $\alpha_{m}^{n}=\alpha^{m} \delta_{n 0}$. In this case, $N=N_{1}, N_{2}=0$ and $A_{0}^{q}=\frac{1}{q !}$. The function $M_{\psi}$ equals $M_{N}($ recall (6.37)),

$$
\begin{gathered}
M_{\psi}(\boldsymbol{\omega})=\sum_{m=0}^{N}\left|\alpha^{m}\right|^{2}\left(h_{n}^{m}(\rho)\right)^{2}=M_{N}(\rho), \\
\rho=\|\boldsymbol{\omega}\| .
\end{gathered}
$$

$$
\begin{aligned}
\psi_{N}^{0}(\mathbf{x}) & =\sum_{m=0}^{N} \frac{1}{\sqrt{m !}} \rho^{m} e^{-\frac{\rho^{2}}{2}} \frac{e^{-i m \phi}}{\sqrt{2 \pi}}=\frac{1}{\sqrt{2 \pi}} \sum_{m=0}^{N} \frac{(\bar{z})^{m}}{\sqrt{m !}} e^{-\frac{|z|^{2}}{2}} \\
= & \frac{1}{\sqrt{2 \pi}} \sum_{m=0}^{N}\left(-\frac{1}{2}\right)^{m} \frac{\left(\frac{\partial}{\partial z}\right)^{m}}{\sqrt{m !}} e^{-\frac{|z|^{2}}{2}} \quad z=r e^{i \phi} .
\end{aligned}
$$

The (up to phase factors unique) solution $\psi_{N}^{0}$ of (6.50) is now given by $\left(\alpha_{m}=1\right.$ for all $\left.m\right)$

This series converges uniformly on compacta, but not in the $\mathbb{L}_{2}$-sense. The real part of this wavelet cor- 

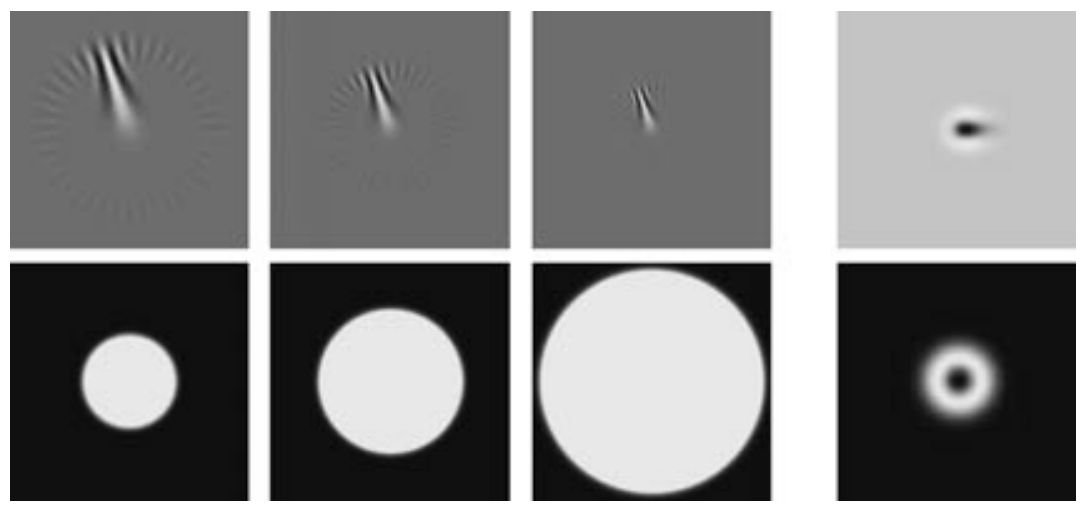

Fig. 10. Top row: 1-3; example of line-detector wavelets $\psi$ in practice (discrete impulse response at $\theta=(13 / 20) \pi)$ at different scales For the sake of illustration, the plots of the kernels are zoomed in by a factor of 2. Example of kernel developed for catheter endpoint detection. Bottom row: the corresponding functions $M_{\psi}$. Notice that the line detectors have better $M_{\psi}$ and thereby induce a more stable exact reconstruction than the catheter endpoint detector.

responds to the wavelet first proposed by Kalitzin [21] as a line detector in medical images. The imaginary part is a good edge detector. For plots of the graph of wavelet $\psi_{N}$ for several values of $N$, see Fig. 6.2.1 and Fig. 10. The wavelets $\psi_{N}^{0}$ have minimal uncertainty in the spatial and frequency domain, in the sense that

$$
\begin{gathered}
\int_{\mathbb{R}^{2}}\|\mathbf{x}\|^{2} \frac{\left|\psi_{N}^{0}\right|^{2}}{\left\|\psi_{N}^{0}\right\|_{\mathbb{L}_{2}\left(\mathbb{R}^{2}\right)}^{2}} d \mathbf{x} \\
\times \int_{\mathbb{R}^{2}}\|\boldsymbol{\omega}\|^{2} \frac{\left|\mathscr{F} \psi_{N}^{0}(\boldsymbol{\omega})\right|^{2}}{\left\|\mathscr{F}\left(\psi_{N}^{0}\right)\right\|_{\mathbb{L}_{2}\left(\mathbb{R}^{2}\right)}^{2}} d \boldsymbol{\omega} \geq \frac{1}{4} \text { for all } N \in \mathbb{N}
\end{gathered}
$$

holds with equality. The basis functions $z \mapsto \frac{z^{m}}{\sqrt{m !}} e^{-\frac{|z|^{2}}{2}}$ are 2D-minimal uncertainty states with $E(\boldsymbol{\omega})=0$ analogously to the Gabor filters in ID-signal analysis. For details, we refer to our technical report [11] Section 7.5, pp. 225-228, where we considered minimal uncertainty states with respect to the Heisenberg group (which is mentioned above and which is most common in image analysis), the Euclidean motion group and the similitude group (which are actually more relevant for wavelet transformations including orientation).

\section{Practical Aspects:}

The cutoff index $N$ has a practical upper bound because of sampling. If $N$ increases, the reconstruction will become better, but if we choose $N$ too large, the wavelet behaves badly along $\phi=0$ (see Fig. 9).

We stress that $\psi_{N}^{0}$ is essentially different than the proper wavelets constructed in the previous subsection: The wavelet $\psi_{N}^{0}$ is clearly not an approximation of the identity as $N \longrightarrow \infty$, whereas the wavelets constructed in the previous section are approximations of the identity as $\boldsymbol{\rho} \longrightarrow \infty$. Therefore, the simple approximative reconstruction from an orientation score $U_{f}$ constructed by $\psi_{N}^{0}$ to image $f$, by integration over the angles only (see (6.40)), is not possible. Thus, we must use the exact inverse given by (4.31) or (5.36) as a close approximative reconstruction, where we notice that $\tilde{\psi}_{0}^{N}$ in contrast to $\psi_{N}^{0}$ is an approximation of the identity.

The size of the wavelet $\psi_{N}^{0}$ can be controlled by dilation, $\mathbf{x} \mapsto\left(\mathscr{D}_{\sigma} \psi_{N}^{0}\right)(\mathbf{x})=\frac{1}{\sigma} \psi_{0}^{N}(\mathbf{x} / \sigma)$. This does effect $M_{\psi}$, since $\mathscr{D}_{\sigma} \mathscr{F}=\mathscr{D}_{1 / \sigma} \mathscr{F}$, but, for $N$ sufficiently large, stability remains guaranteed. Moreover, for large $N$, the wavelet can be smoothed by convolving the wavelet with a relatively small Gaussian kernel:

$$
\begin{gathered}
\psi_{N, s}^{0}(\mathbf{x})=\left(G_{s} * \psi_{N}^{0}\right)(\mathbf{x}) \\
=e^{-(1 / 2)\left|\frac{z}{\mathbf{\rho}}\right|^{2} \beta(\boldsymbol{\rho}, s)}\left(\mathscr{D}_{\alpha(\boldsymbol{\rho}, s)} \psi_{N}^{0}\right)(z), \quad z=x+i y,
\end{gathered}
$$

where $\alpha(\boldsymbol{\rho}, s)=1+\frac{s}{\boldsymbol{\rho}^{2}} \approx 1$ and $\beta(\boldsymbol{\rho}, s)=\frac{1}{\frac{\boldsymbol{\rho}^{2}}{s}+2+\frac{s}{\boldsymbol{\rho}^{2}}}=$ $O\left(\frac{s}{\boldsymbol{\rho}^{2}}\right)$ and $\frac{s}{\boldsymbol{\rho}^{2}} \ll 1$. It is easily verified that $\psi \mapsto G_{s} *$ $\psi$ implies $\tilde{\psi} \mapsto G_{2 s} * \tilde{\psi}$; so as long as the scale s is relatively small, the Gaussian convolution of the wavelet is harmless for the stability of the (re)construction.

For more examples of proper wavelets expanded in eigen functions of the Harmonic oscillator, we refer to [11] Section 7.5, pp. 223-225. 


\section{ORIENTATION SCORES ON 3D IMAGES}

In this section, we generalize our results on the construction of and reconstruction from orientation scores of 3D-images $f \in \mathbb{L}_{2}^{\boldsymbol{\rho}}\left(\mathbb{R}^{3}\right)$. Although some generalizations are straightforward, there arise some difficulties that did not arise in the 2D case. First of all, $S O(3)$ is not commutative, so the $S O(3)$-irreducible representations are not one dimensional. Secondly, in practice, one is mainly interested in constructing orientation scores by "cigar-shaped" wavelets, i.e., wavelets that are invariant under the stabilizer of the north pole, which brings us to the 2-sphere $S^{2}=\frac{S O(3)}{S O(2)}$. Thirdly, it is not obvious which discrete subgroup of $S O(3)$ to take and thus the question arises of how to store the orientation score, since an equidistant sampling in spherical coordinates does not make sense.

Let $f \in \mathbb{L}_{2}^{\mathbf{\rho}}\left(\mathbb{R}^{3}\right)$ be a band-limited 3D image; then we define its wavelet transform $\mathcal{W}_{\psi}[f] \in \mathbb{C}_{K}^{G}$ by

$$
\begin{gathered}
\mathcal{W}_{\psi}[f](g)=\int_{\mathbb{R}^{3}} \overline{\psi\left(R^{-1}(\mathbf{x}-\mathbf{b})\right)} f(\mathbf{x}) d \mathbf{x}, \\
g=(\mathbf{b}, R) \in G=\mathbb{R}^{3} \times S O(3) .
\end{gathered}
$$

We restrict ourselves to the case where the wavelet $\psi$ is invariant under the stabilizer of the north pole $\mathbf{e}_{z}$, which is the subgroup of $S O(3)$ consisting of all rotations around the $z$-axis. So we assume

$$
\psi(R \mathbf{x})=\psi(\mathbf{x}) \text {, for all } R \in \operatorname{Stab}\left(\mathbf{e}_{z}\right) .
$$
tion:

On $S O(3)$, we define the following equivalence rela-

$$
R_{1} \sim R_{2} \Leftrightarrow\left(R_{2}\right)^{-1} R_{1} \in \operatorname{Stab}\left(\mathbf{e}_{z}\right) \equiv S O(2) .
$$

The equivalence classes are the left cosets $[R]=$ $R \operatorname{Stab}\left(\mathbf{e}_{z}\right), R \in S O(3)$. The partition of all equivalence classes will be denoted by $S O(3) / S O(2)$, which is isomorphic to $S^{2}$ and therefore not a group. Rather than using the canonical parameterization given by

$$
\begin{aligned}
& R_{a, \phi}(\mathbf{x})=(\cos \phi) \mathbf{x}+(1-\cos \phi)(\mathbf{a}, \mathbf{x}) \mathbf{a} \\
+ & \sin \phi(\mathbf{a} \times \mathbf{x}), \quad \mathbf{x}, \mathbf{a} \in \mathbb{R}^{3}, \quad \phi \in[0,2 \pi)
\end{aligned}
$$

of $S O(3)$, we will use the well-known Euler angle parameterization $Y: B_{0,2 \pi} \longrightarrow S O(3)$ :

$$
\begin{gathered}
Y(\mathbf{x})=R_{\mathbf{e}_{z}, \gamma} R_{\mathbf{e}_{y}, \beta} R_{\mathbf{e}_{z}, \alpha}, \\
\mathbf{x}=(\alpha \cos \gamma \sin \beta, \alpha \sin \gamma \sin \beta, \alpha \cos \beta)^{T},
\end{gathered}
$$

which gives us directly an explicit isomorphism between $S^{2}$ and $\frac{S O(3)}{S O(2)}$ :

$$
\begin{gathered}
S^{2} \ni \mathbf{n}(\beta, \gamma)=(\cos \gamma \sin \beta, \sin \gamma \sin \beta, \cos \beta)^{T} \\
\longleftrightarrow\left[R_{\mathbf{e}_{z}, \gamma} R_{\mathbf{e}_{y}, \beta}\right] \in \frac{S O(3)}{S O(2)} .
\end{gathered}
$$

Because of our assumption (7.55), we can define the orientation score $U_{f}: \mathbb{R}^{3} \times S^{2} \longrightarrow \mathbb{C}$ corresponding to image $f \in \mathbb{L}_{2}\left(\mathbb{R}^{3}\right)$ by means of

$$
\begin{gathered}
U_{f}(\mathbf{b}, \mathbf{n}(\beta, \gamma))=\mathcal{W}_{\psi}[f]\left(\mathbf{b},\left[R_{\mathbf{e}_{z}, \gamma} R_{\mathbf{e}_{y}, \beta}\right]\right) \\
=\mathcal{W}_{\psi}[f]\left(\mathbf{b}, R_{\mathbf{e}_{z}, \gamma} R_{\mathbf{e}_{y}, \beta}\right) . \\
\text { 7.1. A Simple Approach } \\
\text { of Constructing 3D Proper Wavelets }
\end{gathered}
$$

\subsection{A Simple Approach}

To generalize the idea of constructing proper wavelets by means of suitable decompositions of the unity as illustrated in Example 1 in Section 6.1 to the 3D case, we must discretize $S^{2}$ in disjoint and equal pieces. To this end, we consider an icosahedron $I$, which is the platonic solid with the most ${ }^{16}$ faces $F=20$. It is dual to the dodecahedron and has $V=12$ vertices and $E=V+F-$ $2=30$ edges. Let $S I$ be the discrete subgroup of $S O(3)$ consisting only of rotations that keep $I$ invariant. Let $\mathbf{v}$ be a vertex of $I$; then $|S I * \mathbf{v}|=12$ as $S I$ acts transitively on $I$. Moreover, $\operatorname{Stab}_{S I}(\mathbf{v})=\left\{R_{\mathbf{v}, \beta_{i}}: \beta_{k}=\frac{2 k \pi}{5}, k=0\right.$, $1, \ldots, 4\}$, so we have $|S I|=\left|\operatorname{Stab}_{S I}(\mathbf{v})\right||S I * \mathbf{v}|=5 * 12=$ 60 . So the elements of $S I$ are:

- The 24 rotations by $\frac{2 \pi k}{5}, k=1, \ldots, 4$ about the line passing through a vertex and its opposite.

- The 20 rotations by $\frac{2 \pi k}{3}, k=1,2$ about the line passing through the center $\mathbf{c}_{j}, j=1, \ldots, 12$ of a face and its opposite.

- The 15 rotations by $\pi$ about the center of an edge.

- The identity.

Recall that by our assumption (7.55), we are more interested in a discretization of the two sphere $S^{2}=$ $\frac{S O(3)}{S O(2)}$ than in a discrete subgroup of $S O(3)$. Rather than taking all rotation axes of all elements in $S I$, we consider all (normalized) center points $\tilde{\mathbf{c}}_{i}, i=1, \ldots$, $12 * 2^{q}$ of the triangles $\left\{P_{i_{1}}, P_{i_{2}}, P_{i_{3}}\right\}$ in a $q$-th order (in our example, $q=2$ ) regular tessellation of each face, which are more uniformly distributed over a sphere. For every $i \in\{1, \ldots, n\}$, we define $V_{i}$ as the unique finite

\footnotetext{
16 The platonic solids are the tetrahedron, cube, octahedron, dodecahedron, and icosahedron. Notice to this end that in a convex regular solid in $\mathbb{R}^{3}$ with regular $n$-gon faces, we have $r(n-$ 2) $\pi / n<2 \pi$, where $r$ is the number of faces meeting at every vertex.
} 

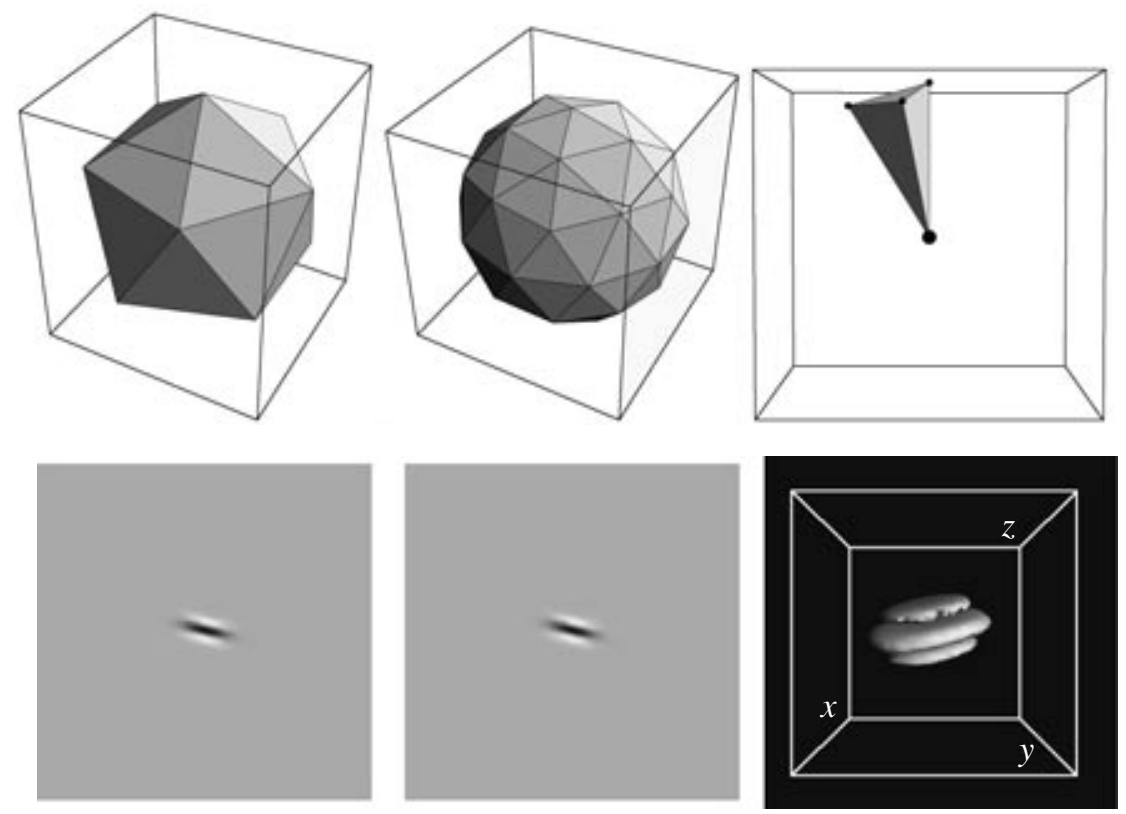

Fig. 11. Top row: Icosahedron, spherical projection of second order tessellated icosahedron, volume $V_{i}$. Bottom row: (for the sake of illustration we zoomed in by factor of 2.5) density plots through XOZ-plane and YOZ-plane of the wavelet $\psi_{i}: \mathbb{R}^{3} \longrightarrow \mathbb{R}\left(\psi_{i}\right.$ sampled at $128 \times 128 \times 128$ pixels, $s=(1 / 2) \sigma^{2}=6,2 * \boldsymbol{\rho}=80$ pixels $)$ given by (7.58), joint contour plot of isosurfaces and $\psi_{i}(\mathbf{x})=$ +0.02 and $\psi_{i}(\mathbf{x})=-0.02$.

volume bounded by the polygons $\left\{O, P_{i_{1}}, P_{i_{2}}\right\}$, $\left\{O, P_{i_{1}}, P_{i_{3}}\right\}$, and $\left\{O, P_{i_{2}}, P_{i_{3}}\right\}$ and the unit sphere. Notice that the unit ball is the disjoint union of these volumes, so $\sum_{i=1}^{12 * 2^{q}} 1_{V_{i}}=1_{B_{0}, 1}$. We now define the smooth wavelets

$$
\begin{aligned}
& \psi_{i}^{\boldsymbol{\rho}}=\mathscr{F}^{-1} {\left[\boldsymbol{\omega} \mapsto\left(G_{s} * 1_{V_{i}}\right)\left(\frac{1}{\boldsymbol{\rho}} \boldsymbol{\omega}\right)\right], } \\
& \boldsymbol{\rho}>0, \quad s>0,
\end{aligned}
$$

which typically detect $2 \mathrm{D}$ ridges/planes (see Fig. 11). They allow a stable (re)construction as $M_{\psi} \simeq 1_{B_{0}, 1}$ and a good approximative reconstruction

$$
f(\mathbf{b}) \approx \sum_{i=1}^{12 * 2^{q}} U_{f}\left(\mathbf{b}, \tilde{\mathbf{c}}_{i}\right)
$$

$$
12 * 2^{q}
$$

since we have $\sum_{i=1} \mathscr{F}\left[\psi_{i}^{\boldsymbol{\rho}}\right](\boldsymbol{\omega})=\left(G_{s} * 1_{B_{0, \boldsymbol{\rho}}}\right)(\boldsymbol{\omega})$, for all $\boldsymbol{\omega} \in \mathbb{R}^{2}$. Notice that for a sufficiently large s, the isosurfaces of $G_{s} * V_{i}$ approach a circular cone, which implies that $\psi_{i}$ is nearly symmetric around the $z$-axis, i.e., nearly satisfies (7.55). Clearly, the wavelet $\psi_{i}$ is the $\mathrm{SD}$-analogue to the 2D wavelet in example 1 in Sec- tion 6.1. However, if one wants to obtain a line detector in $\mathbb{R}^{3}$, one must decompose the unit ball in the Fourier domain with planar structures rather than line structures.

\subsection{General Approach to Constructing 3D Proper Wavelets}

Notice that $\mathbb{L}_{2}\left(\mathbb{R}^{3}\right)=\mathbb{L}_{2}\left(S^{2}\right) \otimes \mathbb{L}_{2}\left((0, \infty), r^{2} d r\right)$. Decompose the angular part $\mathbb{L}_{2}\left(S^{2}\right)$ into irreducible representations:

$$
\mathbb{L}_{2}\left(S_{2}\right)=\underset{l \in \mathbb{N} \cup\{0\}}{\bigoplus} \mathscr{D}^{(l)}
$$

$$
=\bigoplus_{l \in \mathbb{N} \cup\{0\}}\left\langle(\theta, \phi) \mapsto Y_{l}^{m}(\theta, \phi)\right\rangle_{m=-l, \ldots, l},
$$

where the 3D spherical harmonics $Y_{l}^{m} \in \mathbb{L}_{2}\left(S^{2}\right)$ are given by

$$
\begin{gathered}
Y_{l}^{m}(\theta, \phi)=(-1)^{m / 2+|m / 2|} \\
\times \sqrt{\frac{(2 l+1)(l-|m|) !}{4 \pi(l+|m|) !}} P_{l}^{m}(\cos \theta) e^{i m \phi} \\
l \in \mathbb{N}, \quad m=-l, \ldots, l .
\end{gathered}
$$



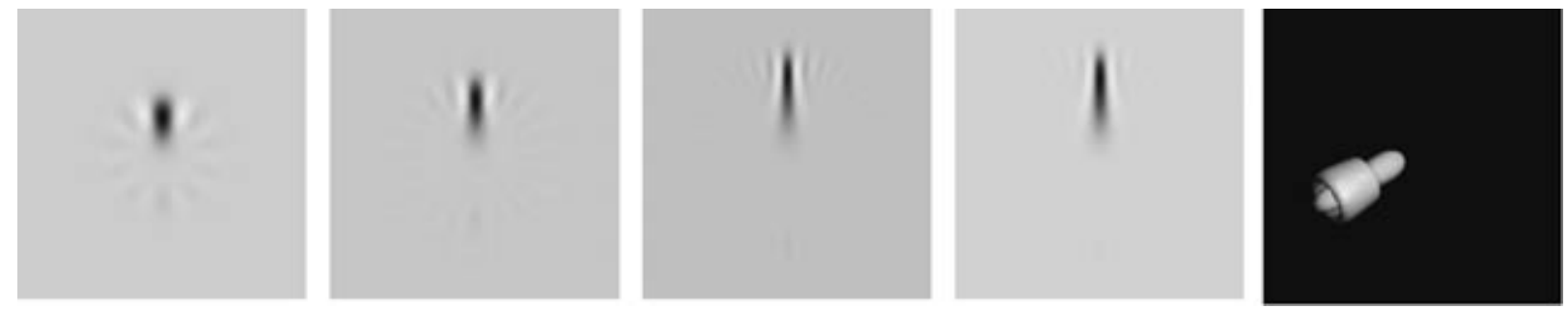

Fig. 12. The wavelet $\psi_{N}^{0,3 \mathrm{D}}$ given by (7.64). From left to right: plots of $\psi_{N}^{0,3 \mathrm{D}}(x, 0, z)$, for $N=10,20,40$ and $G_{s} * \psi_{N=40}^{0,3 \mathrm{D}}=40(x$, $0, z)$, for tiny scale s, and finally a joined 3D plot of the iso-intensity contours of the rotated 3D kernel $\psi_{i}\left(\left(R_{\mathbf{e}_{z}, \phi} R_{\mathbf{e}_{x}, \theta}\right)^{-1} \mathbf{x}\right)=0.5$, $-0.5,\left(\phi \approx-\frac{\pi}{4}\right.$ and $\left.\theta \approx \frac{3 \pi}{4}\right)$.

The orthonormal base $Y_{l}^{m} \otimes g_{n}^{l}$, for definition $g_{n}^{l}$ (see (A.87) in Appendix A), is Fourier invariant (see (A.88)), so we have

$$
\begin{gathered}
\psi=\sum_{n=0}^{\infty} \sum_{l=0}^{\infty} \sum_{m=-l}^{l} \alpha_{m l}^{n} Y_{l}^{m} \otimes g_{n}^{l}, \\
\mathscr{F}[\psi]=\sum_{n=0}^{\infty} \sum_{l=0}^{\infty} \sum_{m=-l}^{l} \alpha_{m l}^{n} i^{l}(-1)^{n+l} Y_{l}^{m} \otimes g_{n}^{l}, \\
\mathscr{F}[\psi]\left(R^{-1} \boldsymbol{\omega}\right)=\sum_{n=0}^{\infty} \sum_{l=0}^{\infty} \sum_{m=-l m^{\prime}=-l}^{l} \sum_{m l}^{l} \alpha_{i}^{n} l^{l}(-1)^{n+l} \\
\times\left(\mathscr{D}^{(l)}(R)\right)_{m^{\prime}}^{m}\left(Y_{l}^{m^{\prime}} \otimes g_{n}^{l}\right)(\varphi, p),
\end{gathered}
$$

with $\alpha_{m l}^{n}=\left(\psi, Y_{l}^{m} \otimes g_{n}^{l}\right)_{\mathbb{L}_{2}\left(\mathbb{R}^{3}\right)}$. It follows from Weyl's Theorem for compact groups (applied to the case $S O(3))$ that

$$
\begin{gathered}
\int_{S O(3)} \overline{\left(\mathscr{D}^{(l)}(R)\right)_{m^{\prime}}^{m}}\left(\mathscr{D}^{(\tilde{l})}(R)\right)_{\tilde{m}^{\prime}}^{\tilde{m}} d R \\
=\frac{1}{2 l+1} \delta^{l \tilde{l}} \delta^{m \tilde{m}} \delta_{m^{\prime} \tilde{m}^{\prime}} 8 \pi^{2} ;
\end{gathered}
$$

and changing the order of integration and summation (Fubini), that

$$
\begin{aligned}
\mathcal{M}_{\psi}(\boldsymbol{\omega})= & 4 \pi \sum_{n=0}^{\infty} \sum_{\tilde{n}=0}^{\infty} \sum_{l=0}^{\infty} \sum_{m=-l m^{\prime}=-l}^{l} \sum_{\frac{1}{2 l} \frac{\overline{\alpha_{m l}^{n}}}{2 l} \alpha_{m l}^{n}(-1)^{n+\tilde{n}}} \\
& \times\left|Y_{l}^{m^{\prime}}(\bar{\theta}, \bar{\phi})\right|^{2} g_{n}^{l}(\rho) g_{\tilde{n}}^{l}(\rho)
\end{aligned}
$$

now it follows from the fact that

PATTERN RECOGNITION AND IMAGE ANALYSIS

$$
\sum_{m=-l}^{l}\left|Y_{l}^{m}(\theta, \phi)\right|^{2}=\frac{(2 l+1)}{4 \pi},
$$

that (7.60) can be simplified to

$$
\begin{aligned}
\mathcal{M}_{\psi}(\boldsymbol{\omega})= & \sum_{n=0}^{\infty} \sum_{\tilde{n}=0}^{\infty} \sum_{l=0}^{\infty} \sum_{m=-l}^{l} \overline{\alpha_{m l}^{\tilde{n}}} \alpha_{m l}^{n}(-1)^{n+\tilde{n}} \\
& \times g_{n}^{l}(\rho) g_{\tilde{n}}^{l}(\rho) .
\end{aligned}
$$

The only spherical harmonics $Y_{l}^{m}$ which are invariant under $\operatorname{Stab}\left(\mathbf{e}_{z}\right)$ are the ones with $m=0$, since $\mathscr{R}_{R_{\mathbf{e}_{z} \beta}}=e^{\beta\left(\mathbf{e}_{z} \times \nabla\right)} Y_{l}^{m}=e^{\beta m i} Y_{l}^{m}$; so under assumption (7.55), the expansion of $\psi$ simplifies to

$$
\begin{gathered}
\psi(\mathbf{x})=\sum_{n=0}^{\infty} \sum_{l=0}^{\infty} \alpha_{l}^{n} g_{n}^{l}(r) Y_{l}^{0}(\theta, \phi), \\
\text { where } \alpha_{l}^{n}=\alpha_{0 l}^{n}, \\
\psi\left(\left(R_{\mathbf{e}_{z}, \gamma} R_{\mathbf{e}_{y}, \beta}\right)^{-1} \mathbf{x}\right) \\
=\sum_{n=0 l=0}^{\infty} \sum_{m^{\prime}=-l}^{\infty} \alpha_{l}^{n}\left[\mathscr{D}^{(l)}\left(R_{\mathbf{e}_{z}, \gamma} R_{\mathbf{e}_{y}, \beta}\right)\right]_{m^{\prime}}^{0} g_{n}^{l}(r) Y_{l}^{m^{\prime}}(\theta, \phi), \\
\mathscr{F}[\psi](\boldsymbol{\omega})=\sum_{n=0}^{\infty} \sum_{l=0}^{\infty} \alpha_{l}^{n}(-1)^{n+l}(i)^{l} g_{n}^{l}(\rho) Y_{l}^{0}(\vartheta, \varphi),
\end{gathered}
$$

for almost every $\mathbf{x} \in \mathbb{R}^{3}$ and $\boldsymbol{\omega} \in \mathbb{R}^{3}$, where we notice that

$$
\begin{gathered}
{\left[\mathscr{D}^{(l)}\left(R_{\mathbf{e}_{z}, \gamma} R_{\mathbf{e}_{y}, \beta}\right)\right]_{m^{\prime}}^{0}=e^{-i m^{\prime} \gamma}} \\
\times \sum_{k=\max \left\{m^{\prime}, 0\right\}}^{\min \left\{l, l+m^{\prime}\right\}} \frac{(-1)^{k}\left(\cos \frac{\beta}{2}\right)^{2 l-2 k+m^{\prime}}\left(\sin \frac{\beta}{2}\right)^{2 k-m^{\prime}}}{k !(l-k) !\left(l+m^{\prime}-k\right) !\left(k-m^{\prime}\right) !}
\end{gathered}
$$



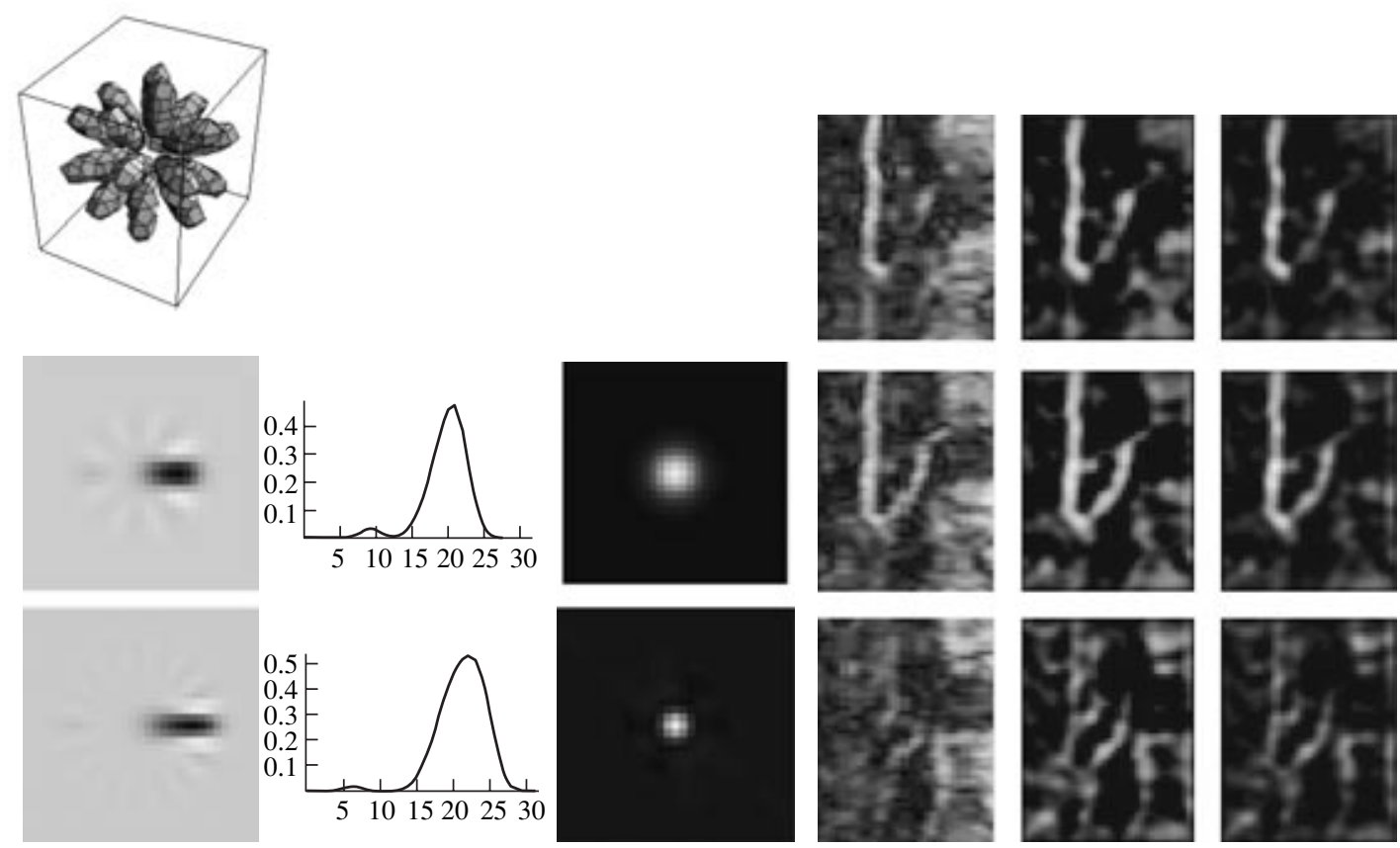

Fig. 13. Left: First row $N=8$, second row $N=16$. From left to right: plots of the wavelet restricted to $y=0$ : $\psi_{N}^{0,3 \mathrm{D}}(x, 0, z)$; the wavelet restricted to $y=0, x=0: \psi_{N}^{0,3 \mathrm{D}}(0,0, z)$; and the restriction of the corresponding $\mathscr{F}^{-1}\left(M_{\psi}\right)=\tilde{\psi}$ to $y=0$, computed by only 20 sampling points, which are the centers $\mathbf{c}_{i}$ of the triangular faces of icosahedron. Right: example of medical application, enhancement of Adam-Kiewicz vessel, in 3D MRI-image by means of power enhancement on the orientation score (see (8.79)) constructed with wavelet, $N=8$, illustrated in the left (upper row).

$$
=\frac{(-1)^{m^{\prime}} Y_{l}^{m^{\prime}}(\beta, \gamma)}{\sqrt{\frac{(2 l+1)}{4 \pi}}} .
$$

The expansion of $M_{\psi}$ now simplifies to

$$
\begin{gathered}
M_{\psi}(\boldsymbol{\omega})=\sum_{l=0}^{\infty} \sum_{n=0}^{\infty} \sum_{\tilde{n}=0}^{\infty}(-1)^{n+\tilde{n}} \overline{\alpha_{l}^{n}} \alpha_{l}^{\tilde{n}} g_{n}^{l}(\rho) g_{\tilde{n}}^{l}(\rho), \\
\text { for all } \boldsymbol{\omega} \in \mathbb{R}^{3},
\end{gathered}
$$

which is the direct equivalent to the third equality of (6.49)! Again we consider the case $\alpha_{l}^{n}=\alpha_{l} \delta^{n 0}$, and $M_{\psi}(\boldsymbol{\omega})$ given by (6.37); then following by $\left(g_{n}^{l}(\boldsymbol{\rho})\right)^{2}=$ $\frac{1}{\Gamma(l+3 / 2)} e^{-\rho^{2}} \rho^{2 l}$ and the Taylor expansion of $e^{\rho^{2}}$, we find the solutions $\left|\alpha_{l}\right|=\sqrt{\frac{\Gamma(l+3 / 2)}{\Gamma(l+1)}}$. In particular, $\alpha_{l}=$ $\sqrt{\frac{\Gamma(l+3 / 2)}{\Gamma(l+1)}}$ gives the wavelet

$$
\psi_{N}^{0.3 D}(\mathbf{x})=\sum_{l=0}^{N} \frac{1}{\sqrt{l} !} r^{l} e^{-\frac{r^{2}}{2}} Y_{l}^{0}(\theta, \phi),
$$

where $Y_{l}^{0}(\theta, \phi)=P_{l}^{0}(\cos \theta) \sqrt{\frac{2 l+1}{4 \pi}}$ is independent of $\phi$. So we have found the SD analogue of the 2D linedetecting wavelet (6.53) (compare Fig. 12 to Fig. 10). We stress the practical advantage of the steerability property of our basis functions (7.61) for computing the orientation score:

$$
\begin{gathered}
U_{f}(\mathbf{b}, \mathbf{n}(\beta, \gamma))=\left(\mathscr{\mathscr { T }}_{\gamma, \beta} \check{\psi}, f\right)_{\mathbb{L}_{2}\left(\mathbb{R}^{3}\right)} \\
=\sum_{n=0}^{\infty} \sum_{l=0}^{\infty} \sum_{m^{\prime}=-l}^{l} \alpha_{l}^{n}\left[\mathscr{D}^{(l)}\left(R_{\mathbf{e}_{z}, \gamma} R_{\mathbf{e}_{y}, \beta}\right)\right]_{m^{\prime}}^{0} \\
\quad \times\left(g_{n}^{l} \otimes Y_{l}^{m^{\prime}}, f\right)_{\mathbb{L}_{2}\left(\mathbb{R}^{3}\right)},
\end{gathered}
$$

which is of course useful, since once coefficients $\left(g_{n}^{l} \otimes\right.$ $\left.Y_{l}^{m^{\prime}}, f\right)_{\mathbb{L}_{2}\left(\mathbb{R}^{3}\right)}$ are computed it is easy to compute $U_{f}(\mathbf{b}$, $\mathbf{n}(\beta, \gamma))$ for a large number of angles $\gamma, \beta$. For elongated structure detection via 3D orientation scores, see Figs. 13 and 14. Another practical advantage of the decomposition in spherical harmonics is that it becomes fairly easy to compute the $\alpha$-scale spaces $W_{f}^{\alpha}(\mathbf{b}, \mathbf{n}(\beta, \gamma), s)[13]$ of the spherical functions $U_{f}(\mathbf{b}, \cdot): S^{2} \longrightarrow \mathbb{R}$ for all $\mathbf{b} \in \mathbb{R}^{3}$ fixed; i.e., $W_{f}^{\alpha}(\mathbf{b}, \mathbf{n}(\beta$, 


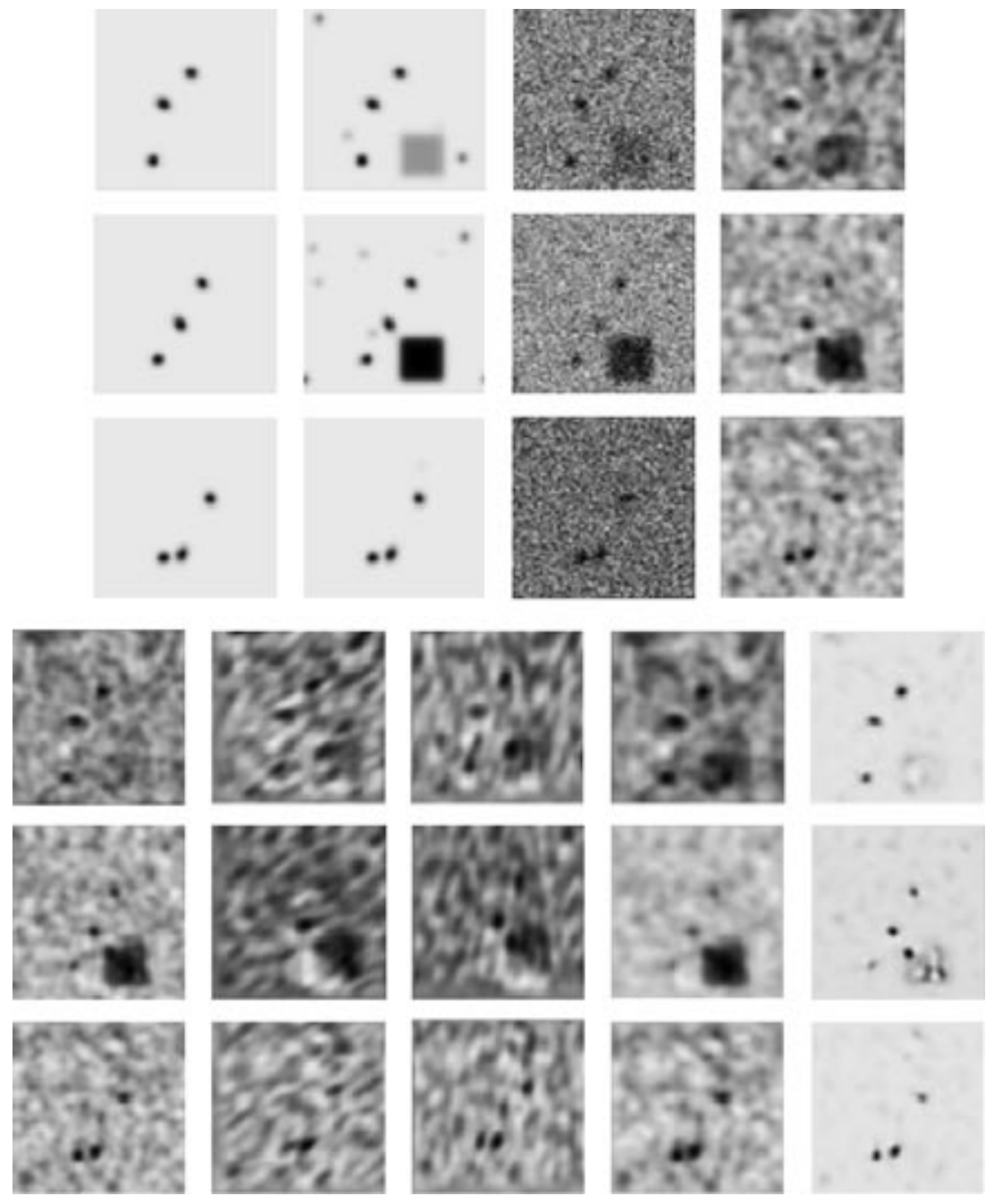

Fig. 14. Illustration of robust line enhancement in $3 \mathrm{D}$ images via orientation scores. SD images $(64 \times 64 \times 64)$ illustrated only by three different 2D cuts (along $x y$ plane, on $z=2,12,22$ ). Orientation scores are constructed with proper wavelet $\psi_{N=16}^{0,3 \mathrm{D}}$ illustrated in Figs. 12 and 13. First three rows: first column original image of a straight line and two circular spirals. Parameterized by, respectively, $(10+(0.2) t, 20+(0.2) t, t),\left(32+10 \cos \left(\frac{2 \pi t}{128}\right), 32+10 \sin \left(\frac{2 \pi t}{128}\right), t\right)$, and $\left(20+12 \cos \left(\frac{2 \pi t}{128}\right), 20+12 \sin \left(\frac{2 \pi t}{80}\right), t\right)$. In the second column we added other geometrical structures, some spots and a cube. In the third column, we obtained $f_{1}$ by adding strong Gaussian distributed noise on the grey values and in the fourth column the approximative reconstruction. In the bottom three rows, we added strongly correlated noise, with the following (from left to right): $2 \mathrm{D}$ cuts of original SD image $f_{2}=f_{1}+$ noise, two elements (which are SD images) in its orientation score $U_{f_{2}}\left(\cdot, \tilde{\mathbf{c}}_{i}\right)=\mathcal{W}_{\psi}\left[f_{2}\right]\left(\cdot, \tilde{\mathbf{c}}_{i}\right)$, with $\tilde{\mathbf{c}}_{2} \approx(-0.19,0.58,0.79), \tilde{\mathbf{c}}_{7} \approx(-0.30,0.93,0.19)$, the approximative reconstruction $f_{2} * \tilde{\psi}$, and the processed image after simple power enhancement (see (8.79)) in score. We did not use any thresholding on the grey values (which is by definition an ill-posed operation).

$\gamma), s)$ is the unique solution $W_{f}^{\alpha}(\mathbf{b}, \mathbf{n}(\beta, \gamma), s)$ of the evolution (diffusion iff $\alpha=1$ ) system

$$
\left\{\begin{array}{l}
\frac{\partial W^{\alpha}}{\partial s}=-\left(-\Delta_{L B}\right)^{\alpha} W, \quad \alpha \in\left[\frac{1}{2}, 1\right] \\
\lim _{s \downarrow 0} W^{\alpha}(\mathbf{b}, \cdot, s)=U_{f}(\mathbf{b}, \cdot) \text { in } \mathbb{L}_{2}\left(S^{2}\right) \text {-sense, }
\end{array}\right.
$$

where $\Delta_{L B}=\frac{\partial^{2}}{\partial \beta^{2}}+\frac{\cos \beta}{\sin \beta} \frac{\partial}{\partial \beta}+\frac{1}{(\sin \beta)^{2}} \frac{\partial^{2}}{\partial \gamma^{2}}$ is the

Laplace-Beltrami operator on $S^{2}$. The unique solution (recall (7.62)) of this evolution problem is simply given by 


$$
\begin{gathered}
W_{f}^{\alpha}(\mathbf{b}, \mathbf{n}(\beta, \gamma), s) \\
=\sum_{n=0}^{\infty} \sum_{l=0}^{\infty} \sum_{m^{\prime}=-l}^{l} \alpha_{l}^{n}\left[\mathscr{D}^{(l)}\left(R_{\mathbf{e}_{z}, \gamma} R_{\mathbf{e}_{y}, \beta}\right)\right]_{m^{\prime}}^{0} \\
\times\left(g_{n}^{l} \otimes Y_{l}^{m^{\prime}}, f\right)_{\mathbb{L}_{2}\left(\mathbb{R}^{3}\right)} e^{-(l(l+1))^{\alpha} s} .
\end{gathered}
$$

\section{IMAGE ENHANCEMENT BY MEANS OF LEFT-INVARIANT OPERATIONS ON ORIENTATION SCORES}

Now that we have constructed an orientation score $U_{f}$ from image $f$, such that it allows a well-posed reconstruction of $f$ from orientation score $U_{f}$, one can think of suitable operations on the orientation scores.

Let $\psi$ be a proper wavelet; then there exists a 1-to-1 correspondence between bounded operators $\Phi \in$ $\mathscr{B}\left(\mathbb{C}_{K}^{G}\right)$ on orientation scores and bounded operators $\Upsilon \in \mathscr{B}_{(}\left(\mathbb{L}_{2}\left(\mathbb{R}^{d}\right)\right)$ on band-limited images,

$$
\begin{gathered}
\Upsilon[f]=\left(\mathcal{W}_{\psi}^{\boldsymbol{\rho}}\right) *\left[\Phi\left[\mathcal{W}_{\psi}^{\boldsymbol{\rho}}[f]\right]\right], \\
f \in \mathbb{L}_{2}^{\mathbf{\rho}}\left(\mathbb{R}^{d}\right),
\end{gathered}
$$

which allows us to relate operations on orientation scores to operations on images in a robust manner ${ }^{17}$.

Recall (Theorem 4.4) and Corollary 2 that $\mathbb{C}_{K}^{G}$ is the space of orientation scores as a closed linear subspace of $\mathbb{H}_{\psi}$, which is a vector subspace of $\mathbb{L}_{2}(G)$. For proper wavelets, we have (approximate) $\mathbb{L}_{2}$-norm preservation, and thus we have $\mathbb{L}_{2}(G) \cong \mathbb{U}_{\psi}$. In this section, we set $\mathbb{H}_{\psi}=\mathbb{L}_{2}(G)$ to avoid technical (irrelevant) subtleties concerning approximations.

Let $\Phi: \mathbb{L}_{2}(G) \longrightarrow \mathbb{L}_{2}(G)$ be some bounded operator on $\mathbb{L}_{2}(G)$; then the range of the restriction of this operator to the subspace $\mathbb{C}_{K}^{G}$ of orientation scores need not be contained in $\mathbb{C}_{K}^{G}$, i.e., $\Phi\left(U_{f}\right)$ need not be the orientation score of an image. The adjoint mapping of $\mathcal{W}_{\psi}^{\rho}$ : $\mathbb{L}_{2}\left(\mathbb{R}^{d}\right) \longrightarrow \mathbb{L}_{2}(G)$ (recall definition 5.2 ) is given by

$$
\begin{gathered}
\left(\mathcal{W}_{\psi}^{\mathbf{\rho}}\right) *(U)=\int_{G} \boldsymbol{U}_{g} \psi U(g) d \mu_{G}(g), \\
U \in \mathbb{L}_{2}(G) .
\end{gathered}
$$

\footnotetext{
17 This can be compared with image processing via the Fourier domain. By the Plancherel Formula $\|f\|_{\mathbb{L}_{2}\left(\mathbb{R}^{2}\right)}^{2}=\|\mathscr{F}[f]\|_{\mathbb{L}_{2}\left(\mathbb{R}^{2}\right)}^{2}$, the Fourier transform $\mathscr{F}: \mathbb{L}_{2}\left(\mathbb{R}^{2}\right) \longrightarrow \mathbb{L}_{2}\left(\mathbb{R}^{2}\right)$ has a stable inverse $\mathscr{F}^{-1}=\mathscr{F}^{*}$, allowing image processing via the Fourier domain.
}

The operator $\mathbb{P}_{\psi}=\mathcal{W}_{\psi}^{\boldsymbol{\rho}}\left(\mathcal{W}_{\psi}^{\boldsymbol{\rho}}\right) *$ is the orthogonal projection on the space of orientation scores $\mathbb{C}_{K}^{G}$. This projection can be used to decompose the manipulated orientation score:

$$
\Phi\left(U_{f}\right)=\mathbb{P}_{\psi}\left(\Phi\left(U_{f}\right)\right)+\left(I-\mathbb{P}_{\psi}\right)\left(\Phi\left(U_{f}\right)\right) .
$$

Notice that the orthogonal complement $\left(\mathbb{C}_{K}^{G}\right)^{\perp}$, which equals $\mathscr{R}\left(I-\mathbb{P}_{\psi}\right)$, is exactly the nil-space of $\left(\mathcal{W}_{\psi}^{\boldsymbol{\rho}}\right)^{*}$, so

$$
\begin{gathered}
{\left[\left(\mathcal{W}_{\psi}^{\boldsymbol{\rho}} * \circ \Phi \circ \mathcal{W}_{\psi}^{\boldsymbol{\rho}}\right)\right](f)} \\
=\left[\left(\mathcal{W}_{\psi}^{\boldsymbol{\rho}}\right) * \circ \mathbb{P}_{\psi} \circ \Phi \circ \mathcal{W}_{\psi}^{\boldsymbol{\rho}}\right](f),
\end{gathered}
$$

for all $f \in \mathbb{L}_{2}^{\mathbf{\rho}}\left(\mathbb{R}^{d}\right)$ and all $\Phi \in \mathscr{B}\left(\mathbb{L}_{2}(G)\right)$.

Definition 8.3. An operator $\Phi: \mathbb{L}_{2}(G) \longrightarrow \mathbb{L}_{2}(G)$ is left-invariant iff

$$
\begin{gathered}
\qquad\left[\mathscr{L}_{h} f\right]=\mathscr{L}_{h}[\Phi f], \\
\text { for all } h \in G, \quad f \in \mathbb{L}_{2}\left(\mathbb{R}^{2}\right),
\end{gathered}
$$

where the left regular action $\mathscr{L}_{g}$ (also known as the shift-twist transformation [30]) of $g \in G$ onto $\mathbb{L}_{2}(G)$ is given by

$$
\begin{gathered}
\mathscr{L}_{g} \psi(h)=\psi\left(g^{-1} h\right)=\psi\left(R_{\theta}^{-1}\left(\mathbf{b}^{\prime}-\mathbf{b}\right), \theta^{\prime}-\theta\right), \\
\text { with } g=(\mathbf{b}, \theta) \in G, \quad h=\left(\mathbf{b}^{\prime}, \theta^{\prime}\right) \in G .
\end{gathered}
$$

Theorem 8.6. Let $\Phi$ be a bounded operator on $\mathbb{C}_{K}^{G}$. Then the unique corresponding operator $\Upsilon$ on $\mathbb{L}_{2}\left(\mathbb{R}^{d}\right)$, which is given by $\Upsilon[f]=\left(\mathcal{W}_{\psi}^{\boldsymbol{\rho}}\right)^{*} \circ \Phi \circ \mathcal{W}_{\psi}^{\boldsymbol{\rho}}[f]$ is Euclidean invariant, i.e., $\mho_{g} \Upsilon=\Upsilon u_{g}$ for all $g \in G$ if and only if $\Phi$ is left-invariant, i.e., $\mathscr{L}_{g} \Phi=\Phi \mathscr{L}_{g}$ for all $g \in G$.

Proof. First, we note that

$$
\mathcal{W}_{\psi}^{\boldsymbol{\rho}} \mathcal{U}_{g}=\mathscr{L}_{g} \mathcal{W}_{\psi}^{\boldsymbol{\rho}} \text {, for all } g \in G,
$$

since $\mathscr{W}_{\psi}^{\rho}\left[\mathcal{U}_{g}[f]\right](h)=\left(\cup_{h} \psi, \quad u_{g} f\right)_{\mathbb{L}_{2}\left(\mathbb{R}^{2}\right)}=$ $\left(\mathcal{U}_{g^{-1} h} \psi, f\right)_{\mathbb{L}_{2}\left(\mathbb{R}^{2}\right)}=\mathscr{L}_{g}\left[\mathcal{W}_{\psi}^{\rho}[f]\right](h)$. Moreover,

$$
\begin{gathered}
\left(\mathcal{W}_{\psi}^{\boldsymbol{\rho}} \mathcal{U}_{g} f, U\right)_{\mathbb{L}_{2}(G)}=\left(\mathscr{L}_{g} \mathcal{W}_{\psi}^{\boldsymbol{\rho}} f, U\right)_{\mathbb{L}_{2}(G)} \\
\Leftrightarrow\left(f, \mathcal{U}_{g^{-1}}\left(\mathcal{W}_{\psi}^{\boldsymbol{\rho}}\right) * U\right)_{\mathbb{L}_{2}(G)}=\left(f,\left(\mathcal{W}_{\psi}^{\boldsymbol{\rho}}\right) * \mathscr{L}_{g^{-1}} U\right)_{\mathbb{L}_{2}(G)}
\end{gathered}
$$

for all $U \in \mathbb{L}_{2}(G), f \in \mathbb{L}_{2}^{\boldsymbol{\rho}}\left(\mathbb{R}^{d}\right), g \in G$, and thus we have

$$
\left(\mathcal{W}_{\psi}^{\boldsymbol{\rho}}\right) * \boldsymbol{U}_{g}=\mathscr{L}_{g}\left(\mathcal{W}_{\psi}^{\boldsymbol{\rho}}\right)^{*}, \text { for all } g \in G
$$

Now suppose $\Phi$ is left-invariant; then it follows by (8.70) and (8.71) that

$$
\Upsilon\left[\mathcal{U}_{g} f\right]=\left(\mathcal{W}_{\psi}^{\boldsymbol{\rho}}\right) * \circ \Phi \circ \mathcal{W}_{\psi}^{\boldsymbol{\rho}} \circ \mathcal{U}_{g}[f]
$$




$$
\begin{aligned}
& =\left(\mathcal{W}_{\psi}^{\boldsymbol{\rho}}\right) * \circ \Phi \circ \mathscr{L}_{g} \circ \mathcal{W}_{\psi}^{\boldsymbol{\rho}}[f] \\
& =\left(W_{\psi}^{\mathbf{\rho}}\right) * \circ \mathscr{L}_{g} \circ \Phi \circ \mathcal{W}_{\psi}^{\boldsymbol{\rho}}[f] \\
& =\boldsymbol{U}_{g} \circ\left(\mathcal{W}_{\psi}^{\boldsymbol{\rho}}\right) * \circ \Phi \circ \mathcal{W}_{\psi}^{\boldsymbol{\rho}}[f]=\boldsymbol{U}_{g}[\Upsilon[f]]
\end{aligned}
$$

for all $f \in \mathbb{L}_{2}\left(\mathbb{R}^{2}\right)$ and $g \in G$. So we indeed obtain $\Upsilon u_{g}=u_{g} \Upsilon$ for all $g \in G$.

Now suppose $\Phi$ is left-invariant. Then we again have by $(8.70)$ and (8.71) that

$$
\begin{aligned}
& \left(\mathcal{W}_{\psi}^{\boldsymbol{\rho}}\right) * \circ \Phi \circ \mathscr{L}_{g} \circ \mathcal{W}_{\psi}^{\boldsymbol{\rho}}[f] \\
= & \left(\mathcal{W}_{\psi}^{\boldsymbol{\rho}}\right) * \circ \mathscr{L}_{g} \circ \Phi \circ\left(\mathcal{W}_{\psi}^{\boldsymbol{\rho}}[f],\right)
\end{aligned}
$$

for all $f \in \mathbb{L}_{2}\left(\mathbb{R}^{2}\right)$ and $g \in G$. Now, since the range of $\left.\mathscr{L}_{g}\right|_{\mathbb{C}_{K}^{G}}$ and the range of $\Phi$ are contained in $\mathbb{C}_{K}^{G}$ and since $\left.\left(\mathcal{W}_{\psi}^{\boldsymbol{\rho}}\right) *\right|_{\mathbb{C}_{K}^{G}}=\left(\mathcal{W}_{\psi}^{\boldsymbol{\rho}}\right)^{-1}$, we obtain $\mathscr{L}_{g} \circ \Phi \circ \mathcal{W}_{\psi}^{\boldsymbol{\rho}}=$ $\Phi \circ \mathscr{L}_{g} \circ \mathcal{W}_{\psi}^{\rho}$. Now the final result follows from the fact that the range of $\mathcal{W}_{\psi}^{\rho}$ equals $\mathbb{C}_{K}^{G}: \mathscr{L}_{g} \circ \Phi=\Phi \circ \mathscr{L}_{g}$ for all $g \in G$.

Practical Consequences: The euclidean invariance of $\gamma$ is of great practical importance since the result should not be different if the original image is rotated or translated. So by Theorem 8.6, the only reasonable operations on orientation scores are left-invariant. Moreover, by (8.67) it follows that is not a problem when the mapping $\Phi: \mathbb{C}_{K}^{G} \longrightarrow \mathbb{L}_{2}(G)$ maps an orientation score to an element in $\mathbb{L}_{2}(G) \backslash \mathbb{C}_{K}^{G}$ as long one is aware of the effect that $\mathbb{P}_{\psi} \Phi: \mathbb{C}_{K}^{G} \longrightarrow \mathbb{C}_{K}^{G}$ yields the same result. One can always compute the angle between $\Phi\left(U_{f}\right)$ and $\mathbb{C}_{K}^{G}$ to see how effective the operation $\Phi$ is (In most of our applications the angle was small).

All linear left-invariant kernel operators $\Phi$ : $\mathbb{L}_{2}(G) \longrightarrow \mathbb{L}_{2}(G)$ are $G$-convolution operators. They are given by

$$
\begin{aligned}
& {[\Phi(U)](g)=\int_{G} K\left(h^{-1} g\right) U(h) d h, \quad g=\left(\mathbf{b}, e^{i \theta}\right) } \\
= & \int_{\mathbb{R}^{2}}^{2 \pi} K\left(e^{i\left(\theta-\theta^{\prime}\right)}, R_{\theta^{\prime}}^{-1}\left(\mathbf{b}-\mathbf{b}^{\prime}\right)\right) U\left(\mathbf{b}^{\prime}, e^{i \theta^{\prime}}\right) d \theta^{\prime} d b_{1}^{\prime} d b_{2}^{\prime},
\end{aligned}
$$

for almost every $g=(\mathbf{b}, \theta) \in G$. From the practical point of view (speed), these can be implemented via impulse response followed by taking the $G$-convolution. Before we propose left-invariant operators on orientation scores, we give a brief overview of the interesting geometry within the domain $G$ of orientation scores, which is the Euclidean Motion Group.

\subsection{Geometry of the Euclidean Motion Group}

For any Lie group $G$, the tangent space $T_{e}(G)$ at the unity element equipped with the product

$$
[A, B]=\lim _{t \downarrow 0} \frac{a(t) b(t)(a(t))^{-1}(b(t))^{-1}-e}{t^{2}},
$$

where $t \mapsto a(t)$ resp. $t \mapsto b(t)$ are any smooth curves in $G$ with $a(0)=b(0)=e$ and $a^{\prime}(0)=A$ and $b^{\prime}(0)=B$, is isomorphic to $\mathscr{L}(G)$. $\mathscr{L}(G)$ is the Lie algebra of leftinvariant vector fields on $G$, i.e., all vector fields $\tilde{A}$ on $G$ such that

$$
\tilde{A}_{g} f=\tilde{A}_{e}\left(f \circ L_{g}\right)=\tilde{A}_{e}(h \mapsto f(g h)),
$$

equipped with product

$$
[\tilde{A}, \tilde{B}]_{g} f=\tilde{A}_{g} \tilde{B} f-\tilde{B}_{g} \tilde{A} f .
$$

The isomorphism is given by $A \longleftrightarrow \tilde{A} \Leftrightarrow \tilde{A}_{g}(\phi)=$ $A\left(\phi \circ L_{g}\right)=A(h \mapsto \phi(g h))$ for all smooth $\phi: G \supset O_{g} \longrightarrow$ $\mathbb{R}$ and all $g, h$ in $G$.

In our case, of the Euclidean motion group we have that $T_{e}(G)$ is spanned by $\left\{A_{1}=\mathbf{e}_{\theta}, A_{2}=\mathbf{e}_{\xi}, A_{3}=\mathbf{e}_{\eta}\right\}$ with

$$
\begin{aligned}
\xi & =b_{1} \cos \theta+b_{2} \sin \theta \\
\mathbf{e}_{\xi} & =\cos \theta \mathbf{e}_{b_{1}}+\sin \theta \mathbf{e}_{b_{2}}
\end{aligned}
$$

(in the spatial plane along the measured orientation) and

$$
\begin{aligned}
& \eta=-b_{1} \sin \theta+b_{2} \cos \theta \\
& \mathbf{e}_{\eta}=-\sin \theta \mathbf{e}_{b_{1}}+\cos \theta \mathbf{e}_{b_{2}}
\end{aligned}
$$

(in the spatial plane orthogonal to the measured orientation). The corresponding left (or shift-twist) invariant vector fields are given by

$$
\begin{gathered}
\left\{\tilde{A}_{1}=\partial_{\theta}, \tilde{A}_{2}=\partial_{\xi}=\cos \theta \partial_{b_{1}}+\sin \theta \partial_{b_{2}},\right. \\
\left.\tilde{A}_{3}=\partial_{\eta}=-\sin \theta \partial_{b_{1}}+\cos \theta \partial_{b_{2}}\right\} .
\end{gathered}
$$

It is easily verified that

$$
\left\{\begin{array} { l } 
{ \tilde { A } _ { 3 } = [ \tilde { A } _ { 1 } , \tilde { A } _ { 2 } ] } \\
{ - \tilde { A } _ { 2 } = [ \tilde { A } _ { 1 } , \tilde { A } _ { 3 } ] \text { and } } \\
{ 0 = [ \tilde { A } _ { 2 } , \tilde { A } _ { 3 } ] }
\end{array} \left\{\begin{array}{l}
A_{3}=\left[A_{1}, A_{2}\right] \\
-A_{2}=\left[A_{1}, A_{3}\right] \\
0=\left[A_{2}, A_{3}\right]
\end{array}\right.\right.
$$

which coincides with $A \longleftrightarrow \tilde{A}$. For dimensional consistency, define $X_{1}=\left(\frac{2 \pi}{Z}\right) A_{1}, X_{2}=A_{2}$, and $X_{3}=A_{3}$, where $Z$ is the width of the image domain (so $b_{1}, b_{2} \in$ $[0, Z])$. A group element $g=(\mathbf{b}, \theta)$ can then be parameterized using either coordinates of the first $\left\{\alpha_{i}\right\}_{i=1,2,3}$, or of the second kind $\left\{\beta_{i}\right\}_{i=1,2,3}$ : 


$$
\begin{gathered}
g=\exp \left(\sum_{i=1}^{3} \alpha_{i} X_{i}\right) \\
=\left(\frac{2 \pi \alpha_{1}}{Z}, \frac{Z \alpha_{2}}{2 \pi \alpha_{1}}\left(\cos \left(\frac{2 \pi \alpha_{1}}{Z}\right)-1\right)\right. \\
\quad-\frac{Z \alpha_{3}}{2 \pi \alpha_{1}} \sin \left(\frac{2 \pi \alpha_{1}}{Z}\right), \\
\left.\frac{Z \alpha_{2}}{2 \pi \alpha_{1}} \sin \left(\frac{2 \pi \alpha_{1}}{Z}\right)+\frac{Z \alpha_{3}}{2 \pi \alpha_{1}}\left(\cos \left(\frac{2 \pi \alpha_{1}}{Z}\right)-1\right)\right), \\
=\prod_{i=1}^{3} \exp \left(\beta_{i} X_{i}\right)=\left(\frac{2 \pi \beta_{1}}{Z}, 0,0\right)\left(0, \beta_{2}, 0\right)\left(0,0, \beta_{3}\right) \\
=\left(\frac{2 \pi \beta_{1}}{Z}, \beta_{2} \cos \left(\frac{2 \pi \beta_{1}}{Z}\right)-\beta_{3} \sin \left(\frac{2 \pi \beta_{1}}{Z}\right),\right. \\
\left.\beta_{2} \sin \left(\frac{2 \pi \beta_{1}}{Z}\right)+\beta_{3} \cos \left(\frac{2 \pi \beta_{1}}{Z}\right)\right) .
\end{gathered}
$$

The coordinates of the second kind correspond to $(\theta, \xi, \eta)$, since by $(8.78):\left(\beta^{1} Z, \beta^{2}, \beta^{3}\right)=\left(\theta, R_{-\theta} \mathbf{b}\right)=$ $(\theta, \xi, \eta)$.

\subsection{Basic Left-invariant Operations on Orientation Scores}

In image analysis it is well-known that differential operators used for corner/line/edge/blob detection must be Euclidean invariant. Mostly, such differential invariants are easily expressed in a local coordinate system (gauge coordinates) where in 2D one coordinate axis $($ say $\mathrm{V}$ ) is along the isoline/isophote and the other along the gradient direction (say $w$ ) [18].

Rather than putting these gauge coordinates along isophotes, we propose a local coordinate system along the measured orientation. Note to this end that in some medical image applications, the elongated structures are not along isophotes. So in our orientation scores, $\xi$ and $\eta$ play the role of $v$ and $w$. Moreover, we can differentiate along the direction $\theta$ and obtain directional frequencies.

Besides these local left-invariant operators, we can think of more global left-invariant operators, such as normalization or grey-value transformations to enhance certain elongated structures.

Overview of left-invariant operations on orientation scores: ple

- Monotonic Grey-value transformations. For exam-

$$
\Phi\left(U_{f}\right)=\left(U_{f}-\min _{g}\left\{U_{g}(g)\right\}\right)^{q}, \quad q>0 .
$$

This can be used to enhance the strongly oriented spots in the score and reduce the noise or weakly ori- ented spots in the score (see Fig. 8.2). Note that it does not correspond to a simple grey-value transformation of the original image, since in general we have $f^{q} \neq$ $\left(W_{\psi}^{\mathbf{\rho}}\right) *\left[\left(W_{\psi}[f]\right)^{q}\right]$.

- Normalization. $\left[\Phi\left(U_{f}\right)\right]\left(\mathbf{b}, e^{i \theta}\right)=U_{f}(\mathbf{b}$, $\left.e^{i \theta}\right) /\left(\int_{\mathbb{R}^{2}}\left|U_{f}\left(\mathbf{x}, e^{i \theta}\right)\right|^{p} d \mathbf{x}\right)^{\frac{1}{p}}, p>$ 1. See Fig. 8.1.

- Differential operators constructed from left-invariant vector fields. For example, enhancement of elongated structures via $\Phi\left(U_{f}\right)=\partial_{\eta \eta} U_{f} \partial_{\theta \theta} U_{f} U_{f}$.

- Left-invariant evolution equations generated by left-invariant vector fields. For examples see Subsection 8.3.

\subsection{Evolution Equations Corresponding to Left-Invariant Stochastic Processes on the Euclidean Motion Group}

Just like the well-known Gaussian scale space satisfies the translation and rotation invariance axiom [2], the following linear evolutions on orientation scores are left invariant:

$$
\left\{\begin{array}{l}
\partial_{s} W=A W, \quad a_{i}, b_{i} \in \mathbb{C}, \quad i=1, \ldots, 3 \\
\lim _{s \downarrow 0} W(\cdot, s)=U_{f}(\cdot),
\end{array}\right.
$$

where the generator $A$ acting on $\mathbb{L}_{2}(G)$ is given by (the closure of)

$$
\begin{gathered}
A=\left[-\frac{a_{1}}{Z} \partial_{\theta}-a_{2} \partial_{\xi}-a_{3} \partial_{\eta}\right. \\
\left.+\frac{D_{11}}{Z^{2}}\left(\partial_{\theta}\right)^{2}+D_{22}\left(\partial_{\xi}\right)^{2}+D_{33}\left(\partial_{\eta}\right)^{2}\right]
\end{gathered}
$$

The first order derivatives take care of transport (convection) and the second order derivatives give diffusion. We first consider the case where all $D_{i i}$ 's are zero and the initial condition is a spike-bundle $\delta_{\theta_{0}, \mathbf{b}_{0}}$ (i.e., one oriented particle). This spike will move over time along exponential curves, which are straight-lines in a spatial plane, spirals through $G$, and straight lines along the $\theta$ direction. By introducing the variables $t=$

$s \frac{a_{1}}{Z}, \lambda_{2}=\frac{a_{2}}{a_{1}} Z, \lambda_{3}=\frac{a_{3}}{a_{1}} Z$, Eq. (8.80) reduces to

$$
\left\{\begin{array}{l}
\partial_{t} W=\left[\partial_{\theta}+\lambda_{2} \partial_{\xi}+\lambda_{3} \partial_{\eta}\right] W \\
\lim _{t \downarrow 0} W(\cdot, t)=\delta_{\theta_{0}, \mathbf{b}_{0}}
\end{array} \quad \lambda_{2}, \lambda_{3} \in \mathbb{C} .\right.
$$

Notice that indeed $[s]=1 \longleftrightarrow[t]=1$ and $\left[a_{1}\right]=$ $\left[a_{2}\right]=\left[a_{3}\right]=[$ length $] \longleftrightarrow\left[\lambda_{2}\right]=\left[\lambda_{3}\right]=$ [length $]$. It fol- 

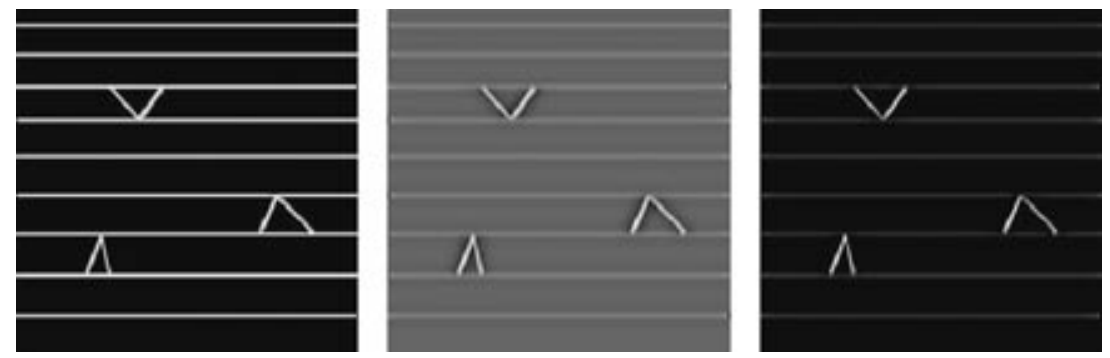

Fig. 15. Illustration of image processing via elementary operation on orientation score. Modeling the visual illusion: normalization of the orientation layers in the orientation scores reveals the most contrasting lines in the triangles.
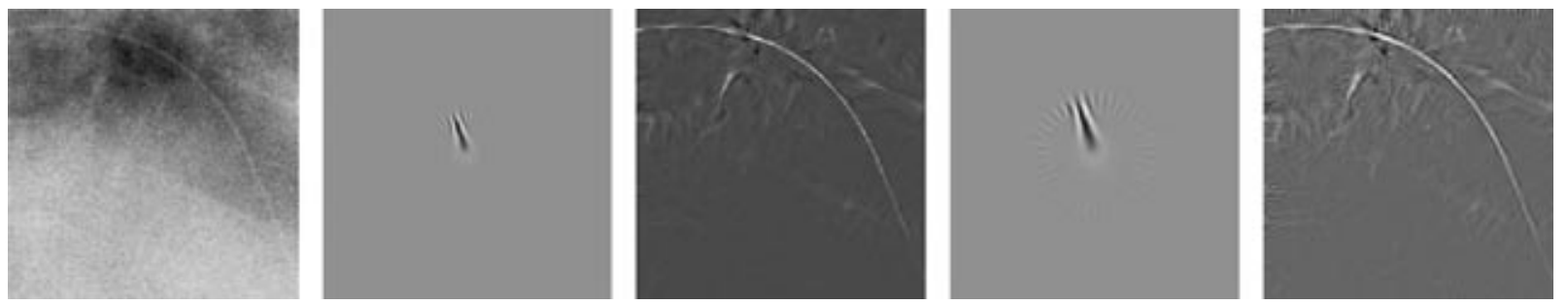

Fig. 16. Illustration of image processing via elementary operation on orientation score. From left to right: 1 , noisy medical image with guide wire; 2-3, small oriented wavelet $\psi$ with corresponding processed image $\tilde{f}=\mathcal{W}_{\psi}^{*}\left[\tilde{U}_{f}\right]$, with $\tilde{U}_{f}=$ $\left(\frac{\Re U_{f}-\min \Re\left(U_{f}\right)}{\max \left\{\Re\left(U_{f}\right)-\min \left(\Re\left(U_{f}\right)\right)\right\}}\right)^{2}$; and 4-5, same as 2-3 with relatively larger kernel $\frac{1}{\sigma} \psi_{N}^{0}\left(\frac{\mathbf{x}}{\sigma}\right)$, where we recall that $\psi_{N}^{0}$ was given by (6.53). For the sake of clarity, the wavelet plots $(2,4)$ are zoomed in with a factor of 2.

lows by equality (8.78) that the orbit of the Dirac distribution at the initial position $\left(\mathbf{b}_{0}, e^{i \theta_{0}}\right)$ is given by

$$
\begin{gathered}
\left(b_{0}^{1}+\lambda_{3}\left(\cos \left(t+\theta_{0}\right)-\cos \left(\theta_{0}\right)\right)\right. \\
+\lambda_{2}\left(\sin \left(t+\theta_{0}\right)-\sin \left(\theta_{0}\right)\right), \\
b_{0}^{2}+\lambda_{3}\left(\sin \left(t+\theta_{0}\right)-\sin \left(\theta_{0}\right)\right)-\lambda_{2}\left(\cos \left(t+\theta_{0}\right)\right. \\
\left.\left.-\cos \left(\theta_{0}\right)\right), e^{i\left(t+\theta_{0}\right)}\right),
\end{gathered}
$$

which is for $a_{1} \neq 0$ a circular spiral with radius $\sqrt{\lambda_{1}^{2}+\lambda_{2}^{2}}$ around central point $\left(-\lambda_{3} \cos \theta_{0}+\lambda_{2} \sin \theta_{0}+b_{0}^{1}\right.$, $-\lambda_{2} \cos \theta_{0}-\lambda_{3} \sin \theta_{0}+b_{0}^{2}$ ), which exactly corresponds to the results from our numerical implementation. The solution of the diffusion problem, i.e., $a_{1}=a_{2}=a_{3}=0$ in (8.81) is a $G$-convolution kernel operator with some positive kernel $K_{s} \in \mathbb{L}_{1}(G)$, which can be sharply estimated from above and below by Gaussian kernels on $G$ (for details see [14]). In the degenerate case $a_{1}=a_{2}=a_{3}=$ $D_{11}=0$, the diffusion boils down to an ordinary spatial convolution for each fixed $\theta$ with an anisotropic Gaussian kernel where the fraction of $D_{22}$ and $D_{33}$ gives the anisotropy factor of Gaussian convolution along $\mathbf{e}_{\xi}$ and $\mathbf{e}_{\eta}$.

The evolution equations given by (8.80) correspond to stochastic processes. For example, the case $a_{1}=a_{3}=0$ and $D_{22}=D_{33}=0$ is the Forward Kolmogorov equation corresponding to the stochastic process known as the direction process ${ }^{18}$ [25]:

$$
\left\{\begin{array}{l}
\bar{\kappa}=\frac{1}{L} \int_{0}^{L} k(s) d s=\frac{1}{L} \int_{0}^{L}|\dot{\theta}(s)| d s \sim \mathcal{N}\left(0, \sigma^{2}\right) \\
\mathbf{x}(s)=\int_{0}^{s}\left(\begin{array}{c}
\cos \theta(\tau) \\
\sin \theta(\tau)
\end{array}\right) d \tau+\mathbf{x}(0) \\
L \sim N E(\alpha),
\end{array}\right.
$$

which is the limit of the following discrete stochastic process:

$$
\left\{\begin{array}{l}
\theta\left(s_{i}+\Delta s\right)=\theta\left(s_{i}\right)+\Delta s \varepsilon, \quad \varepsilon \sim \mathcal{N}\left(0, N \sigma^{2}\right) \\
\mathbf{x}\left(s_{i}+\Delta s\right)=\mathbf{x}\left(s_{i}\right)+\Delta s\left(\begin{array}{c}
\cos \theta\left(s_{i}\right) \\
\sin \theta\left(s_{i}\right)
\end{array}\right) \\
\Delta s=\frac{L}{N}, \text { with } L \sim N E(\alpha) .
\end{array}\right.
$$

${ }^{18}$ In many later works, Mumford's final Fokker-Plank equation, which is physically correct as long as $\sigma^{2}$ is the variance in average curvature $\bar{\kappa}$, is often misformulated in literature, introducing dimensional inconsistencies. For example, [30] and [6], where $\sigma^{2} / 2$ must be $\sigma$. 

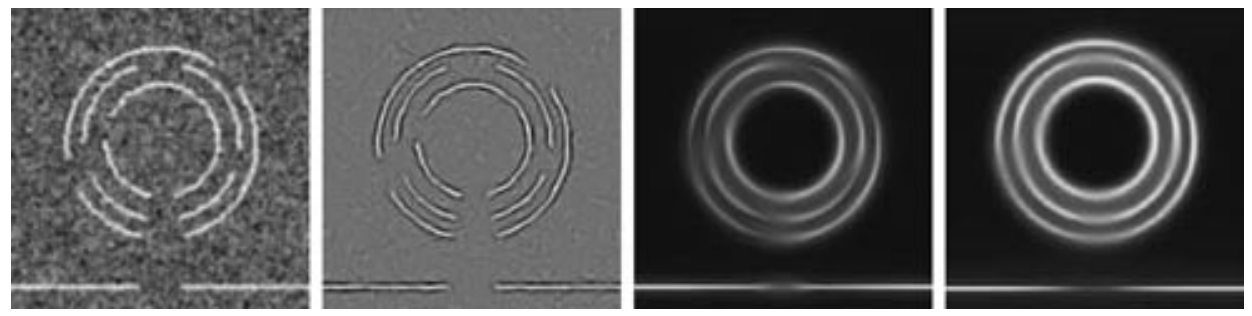

Fig. 17. Example of perceptual organization. From left to right: 1, Original image; 2, detection of elongated structures via orientation scores $U_{f}: \tilde{f}=\mathscr{W}_{\psi}^{*}\left[\tilde{U}_{f}\right]$ with $\tilde{U}_{f}=\left(\frac{\Re U_{f}-\min \Re\left(U_{f}\right)}{\max \left\{\Re\left(U_{f}\right)-\min \left(\Re\left(U_{f}\right)\right)\right\}}\right)^{2} ; 3$, inverse transformation of evolved orientation score $\mathcal{W}_{\psi}^{*}\left[\Phi\left[\tilde{U}_{f}\right]\right]$, where $\Phi$ denotes the shooting process by maintaining curvature and direction; and 4 , inverse transformation of probability density of collision of forward and backward process on orientation score (see (8.82)). In contrast to related work [30], we do not put sources and sinks by hand, but use our orientation scores instead. The only parameters involved are the range of wavelet $t$, decay time $\alpha$, and the stochastic process parameters $b^{1}, b^{2}, b^{3}$ in (8.81).
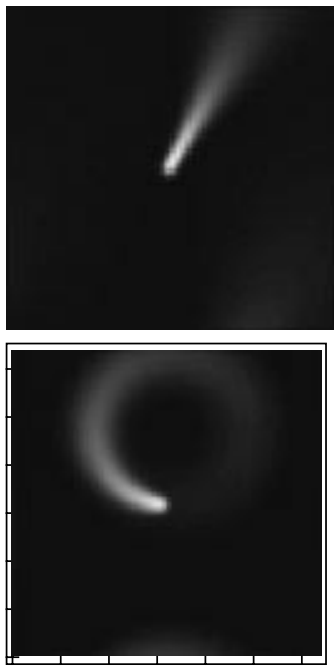

$0 \quad 102030405060$
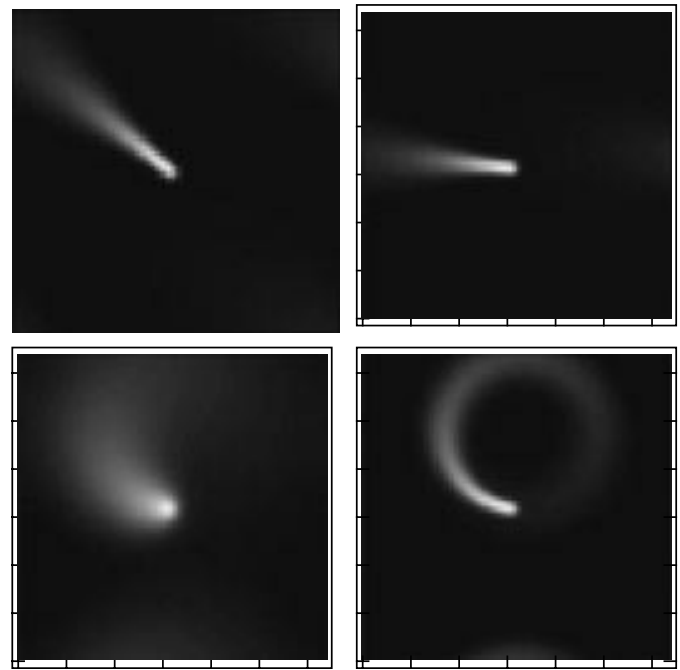

$\begin{array}{lllllll}0 & 10 & 20 & 30 & 40 & 50 & 60\end{array}$

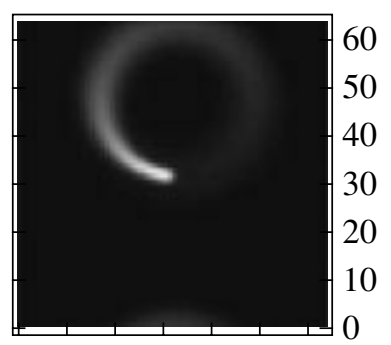

$\begin{array}{llllllll}0 & 10 & 20 & 30 & 40 & 50 & 60\end{array}$

60

50

40

30

20

10

$$
\text { 每 }
$$

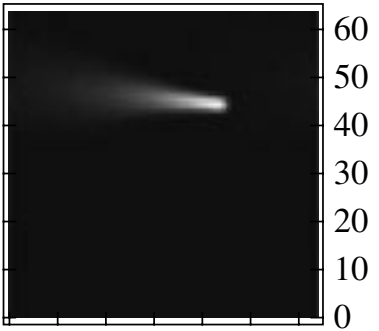

Fig. 18. Top row: Illustrations of output $W$ (equidistantly sampled over $64 \times 64 \times 64$ grid) of directional process (i.e., $a_{i}=\delta_{i 2}, D_{i i}=$ $\sigma \delta_{i 1}$ ), with $\alpha=0.125, \sigma=0.08$, applied on, from left to right, (slightly blurred) initial $64 \times 64 \times 64$ score $\delta_{12} \delta_{32} \delta_{32}, \delta_{26} \delta_{32} \delta_{32}$, $\delta_{32} \delta_{32} \delta_{32}, \delta_{32} \delta_{45} \delta_{45}$. For the sake of illustration, we integrated over the 64 orientations $\theta: \frac{2 \pi}{64} \sum_{k=1}^{64} W\left(\mathbf{b}, \theta_{k}\right) \approx \int_{0}^{2 \pi} W(\mathbf{b}, \theta) d \theta$, and thereby showed 2D visualizations of the $3 D$-impulse responses, which are the Green's functions of the evolution process. Shift-twist invariance of the transformation on the orientation score indeed corresponds to Euclidean invariance of the corresponding transformation on image. Bottom row: impulse responses of $\delta_{32} \delta_{32} \delta_{32}$ impulse with, from left to right, parameter values $\mathbf{a}=\left(a_{1}, a_{2}, a_{3}\right)=$

$(4,1,0),(4,0,1),(4 \sqrt{2}, 1,1)$, and with $\alpha=0.125$, applied on initial $64 \times 64 \times 64$ bundle $\delta_{32} \delta_{32} \delta_{32}$. For the sake of clarity, grey values have been scaled to full range.

Just like scale space theory ${ }^{19}$, a scale space representation $u(\mathbf{x}, s)=G_{s} * f$ can be regarded as an isotropic

${ }^{19}$ In a scale space representation $u(\mathbf{x}, s)=G_{s} * f$, the evolution parameter/scale $s=\frac{\sigma^{2}}{2}$ inherits the physical dimension of the generator $\Delta$ of the corresponding evolution equation $u_{s}=\Delta u$. Scale can be related to time a diffusion constant $D: D t=s=\frac{1}{2} \sigma^{2}$. stochastic process where the distribution of positions of grey-value particles (photons) evolves over time (the well-known Wiener process), evolutions on orientation scores can be considered as stochastic processes where the distribution of positions of oriented photons evolves over time.

The lifetime $T$ of a particle traveling with unit speed (so $T=L$ ) through $G$ is assumed to be negative exponentially distributed $\left(T \sim N E(\alpha)\right.$, i.e., $\left.p(T=t)=\alpha e^{-\alpha t}\right)$, 
with expected lifetime $E(T)=\frac{1}{\alpha}$, because it is memoryless $P(S \leq \tau+s \mid S>\tau)=\frac{P(\tau<S \leq \tau+S)}{P(S>\tau)}=P(S \leq s)$, which must be the case in a Markov process. The probability density of finding an $e^{i \theta}$ oriented particle at position $\mathbf{b}$ is given by

$$
\begin{gathered}
p(g)=p(\mathbf{b}, \theta)=\int_{0}^{\infty} p(\mathbf{b}, \theta \mid T=t) p(T=t) d t \\
=\alpha \int_{0}^{\infty}\left[e^{t A} U_{f}\right](\mathbf{b}, \theta) e^{-t \alpha} d t=-\alpha\left[(A-\alpha I)^{-1} U_{f}\right](\mathbf{b}, \theta) .
\end{gathered}
$$

Consider two independent stochastic processes generated by $A=$ Conv + Diff, where Conv, resp. Diff stands for the convection resp. diffusion part of $A$, given by (8.81), and its adjoint $A^{*}=-$ Conv + Diff. So the direction of shooting particles is opposite and the stochastic behavior is similar in the two processes. The probability-density of collision of particles from these two processes yields the following left-invariant operation (see Fig. 17):

$$
\begin{gathered}
\left(\Phi\left[U_{f}\right]\right)(g)=\left[(A-\alpha I)^{-1} U_{f}\right](g) \\
\times\left[\left(A^{*}-\alpha I\right)^{-1} U_{f}\right](g) .
\end{gathered}
$$

\section{CONCLUSIONS}

Given an image $f \in \mathbb{L}_{2}\left(\mathbb{R}^{d}\right)$, we construct a local orientation score $U_{f}$, which is a complex-valued function on the Euclidean motion group $G$. The corresponding transformation $f \mapsto U_{f}$ is a wavelet transform constructed from an oriented wavelet and a representation of the Euclidean motion group onto $\mathbb{L}_{2}\left(\mathbb{R}^{2}\right)$. Since this representation $\mathcal{U}$ is reducible, the well-known wavelet reconstruction theorem, which allows perfectly wellposed reconstruction, does not apply.

Therefore, we generalized standard wavelet theory by means of reproducing kernel theory. From this generalization, it followed that our wavelet transformation is a unitary mapping between the space of bandlimited images, modeled by $\mathbb{L}_{2}^{\rho}\left(\mathbb{R}^{2}\right)$ and the functional Hilbert space $\mathbb{C}_{K}^{G}$ of orientation scores, which is explicitly characterized. The norm on $\mathbb{C}_{K}^{G}$ explicitly depends on the oriented wavelet $\psi$ via a function $M_{\psi}$, which thereby characterizes the stability of the explicitly described inverse wavelet transformation. As a result, by proper choice of the wavelet $\psi$, the image $f$ can be reconstructed from $U_{f}$ in a robust way. We developed and implemented several approaches to obtaining proper wavelets (which are also good line detectors in practice) in the 2-dimensional case, $d=2$. These results are also explicitly generalized to (and implemented in) the 3 -dimensional case, $d=3$. These proper wavelets give rise to a stable transformation from image to orientation score and vice versa, allowing us to relate operations $\Phi$ on orientation scores to operations $\Upsilon$ on images in a robust way. Finally, we show that operations $\Phi$ must be left-invariant in order to obtain a Euclidean invariant transformation $\Upsilon$ on images. As an example, we observe the probability of collision of particles from two stochastic processes on the Euclidean motion group, which is used to automatically detect elongated structures and to close gaps between them.

APPENDICES

APPENDIX A

\section{THE BOCHINER-HECKE THEOREM AND THE SPECTRAL DECOMPOSITION OF THE HANKEL TRANSFORM}

Theorem A. 7 Let $H$ be a harmonic homogeneous polynomial of degree $m$ in $d$ variables. Let $F$ be an element of $\mathbb{L}_{2}\left((0, r) ; r^{d+2 m-1} d r\right)$; then the Fourier transform of their direct product $(r, \mathbf{x}) \mapsto F(r) H(\mathbf{x})$, which is in $\mathbb{L}_{2}\left(\mathbb{R}^{d}\right)$, is given by

$$
\begin{gathered}
(-i)^{m} H(\boldsymbol{\omega}) \int_{0}^{\infty}(r \rho)^{\left(\frac{d-2}{2}+m\right)} J_{\frac{d-2}{2}+m}(\rho r) F(r) r^{d+2 m-1} d r \\
=(-i)^{m} H(\boldsymbol{\omega}) \rho^{-\left(\frac{d-1}{2}+m\right)}\left[\mathscr{H}_{\frac{d-2}{2}+m} r^{\frac{d-1}{2}+m} F\right](\rho),
\end{gathered}
$$

where $\rho=\|\boldsymbol{\omega}\|$ and $\mathscr{H}_{\frac{d-2}{2}+m}$ the Hankel Transform given by

$$
\begin{aligned}
&\left(\mathscr{H}_{\frac{d-2}{2}+m} \phi\right)(\rho)=\int_{0}^{\infty}(\rho r)^{1 / 2} \phi(r) J_{\frac{d-2}{2}+m}(\rho r) d r \\
& \phi \in \mathbb{L}_{2}((0, \infty)) .
\end{aligned}
$$

The proof can be found in [16] pp. 24-25. The Hankel Transform $\mathcal{H}_{\mu}, \mu=\frac{d-2}{2}+m$, is a unitary map on $\mathbb{L}_{2}((0, \infty), d r)$ and has a complete set of orthonormal eigen functions $\left\{E_{n}^{\mu}\right\}$ given by

$$
\begin{aligned}
r \mapsto E_{n}^{\mu}(r) & =\left(\frac{2 n !}{\Gamma(n+\mu+1)}\right)^{\frac{1}{2}} r^{\mu+\frac{1}{2}} e^{-\frac{r^{2}}{2}} L_{n}^{(\mu)}\left(r^{2}\right), \\
n & =0,1,2, \ldots, r>0,
\end{aligned}
$$


where $L_{n}^{(\mu)}$ is the $n$-th generalized Laguerrre polynomial of type $\mu>-1$,

$$
L_{n}^{(\mu)}(r)=\frac{r^{-\alpha} e^{r}}{n !}\left(\frac{d}{d r}\right)^{n}\left(e^{-r} r^{n+\mu}\right), \quad r>0,
$$

with corresponding eigen values $(-1)^{n}:\left(\mathscr{H}_{\mu} \phi\right)=$ $\sum_{n=0}^{\infty}(-1)^{n}\left(E_{n}^{\mu}, \phi\right)_{\mathfrak{L}_{2}((0, \infty), d r)} E_{n}^{\mu}$. The functions $E_{n}^{\mu}$ are also eigen functions of the operator

$$
-\frac{d^{2}}{d r^{2}}+r^{2}+\frac{\mu^{2}-\frac{1}{4}}{r^{2}}-2 \alpha
$$

with eigen value $4 n+2$, cf. [15] p. 79, which coincides with the fact that the Harmonic oscillator $\|\mathbf{x}\|^{2}-\Delta$ commutes with the Fourier transform.

For $d=2$, we have $\mathbb{L}_{2}\left(\mathbb{R}^{2}\right)=\mathbb{L}_{2}\left(S^{1}\right) \otimes \mathbb{L}_{2}((0, \infty) ; r d r)$ and a Fourier invariant orthonormal base is given by $\left\{Y_{m} \otimes h_{n}^{m}\right\}_{m \in \mathbb{Z}, n \in \mathbb{N} \cup\{0\}}$, where

$$
h_{n}^{m}=r^{-1 / 2} E_{n}^{m}(r),
$$

and $Y_{m}(\phi)=\frac{1}{\sqrt{2 \pi}} e^{i m \phi}$. It now follows by the BochnerHecke Theorem that:

$$
\begin{gathered}
\mathscr{F}\left(Y_{m} \otimes h_{n}^{m}\right)=\mathscr{F}\left(r^{m} Y_{m} \otimes \frac{h_{n}^{m}}{r^{m}}\right) \\
=(-1)^{n}(-i)^{|m|}\left(Y_{m} \otimes h_{n}^{m}\right) \\
=(-1)^{n+m}(i)^{|m|}\left(Y_{m} \otimes h_{n}^{m}\right) .
\end{gathered}
$$

For $d=3$, we have $\mathbb{L}_{2}\left(\mathbb{R}^{2}\right)=\mathbb{L}_{2}\left(S^{1}\right) \otimes \mathbb{L}_{2}((0, \infty) ; r d r)$. All $l$ homogeneous harmonic polynomials are spanned by $\left\{\mathbf{x} \mapsto r^{l} Y_{l}^{m}(\theta, \phi)\right\}_{l=0 \ldots \infty ; m=-l, \ldots, l}$. Define

$$
g_{n}^{l}(r)=r^{-1} E_{n}^{l+\frac{1}{2}}(r), \quad r>0 ;
$$

then $g_{n}^{l} \in \mathbb{L}_{2}((0, \infty) ; r d r)$ are eigen functions of $\rho^{-1} \mathscr{H}_{\frac{1}{2}+m} r^{1}$ with corresponding eigenvalues $(-1)^{n}$. Therefore, it follows by the Bochner-Hecke Theorem that

$$
\mathscr{F}\left(Y_{l}^{m} \otimes g_{n}^{l}\right)=(i)^{l}(-1)^{n+l}\left(Y_{l}^{m} \otimes g_{n}^{l}\right) .
$$

APPENDIX B

\section{PROOF OF THEOREM 4.3}

We will use the following lemma, which gives a useful characterization of the elements of $\mathbb{C}_{K}^{\natural}$ :

Lemma B.4 Let $K$ be a function of positive type on $\square$ and $F$ a complex-valued function on $\square$. Then the func-

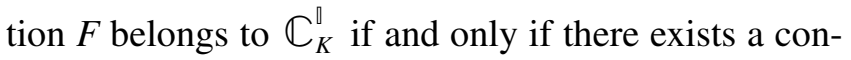
stant $\gamma>0$ such that

$$
\left|\sum_{j=1}^{l} \alpha_{j} \overline{F\left(m_{j}\right)}\right|^{2} \leq \gamma \sum_{k, j=1}^{l} \overline{\alpha_{k}} \alpha_{j} K\left(m_{k}, m_{j}\right),
$$

for all $l \in \mathbb{N}$ and $\alpha_{j} \in \mathbb{C}, m_{j} \in \mathbb{\mathbb { V }}, 1 \leq j \leq l$.

Proof. See [24, Lemma 1.7, p. 31].

From this lemma, it indeed follows that the norm on $\mathbb{C}_{K}^{\natural}$ is given by (4.20).

\section{Proof of Theorem 4.3:}

First we show that $W_{f} \in \mathbb{C}_{K}^{\natural}$ for any element $f \in$ $H=\langle\bar{V}\rangle$ and that $\mathcal{W}$ is bounded (and therefore continuous). If $f \in H$ then

$$
\begin{gathered}
\left|\sum_{j=1}^{l} \alpha_{j} \overline{(\mathscr{W} f)\left(m_{j}\right)}\right|^{2}=\left|\sum_{j=1}^{l} \alpha_{j} \overline{\left(\phi_{m_{j}}, f\right)_{H}}\right|^{2} \\
=\left|\left(\sum_{j=1}^{l} \alpha_{j} \phi_{m_{j}}, f\right)_{H}\right|^{2} \leq\left\|\sum_{j=1}^{l} \alpha_{j} \phi_{m_{j}}\right\|_{H}^{2}\|f\|_{H}^{2} \\
=\left(\sum_{k, j=1}^{l} \overline{\alpha_{k}} \alpha_{j} K\left(m_{k}, m_{j}\right)\right)\|f\|_{H}^{2},
\end{gathered}
$$

for all $l \in \mathbb{N}, \alpha_{1}, \ldots, \alpha_{l} \in \mathbb{C}$, and $m_{1}, \ldots, m_{n} \in \mathbb{Z}$. So $\mathcal{W} f \in \mathbb{C}_{K}^{\square}$ by Lemma B.4 and $\|\mathcal{W} f\|_{\mathbb{C}_{K}^{0}}^{2} \leq\|f\|_{H}^{2}$, by (4.20). Next we prove that $\mathcal{W}$ is an isometry. Because $\left(\phi_{m}, \phi_{m^{\prime}}\right)=K\left(m, m^{\prime}\right), \mathcal{W}$ maps a linear combination $\sum_{i} \alpha_{i} \phi_{m_{i}}$ onto the linear combination $\sum_{i} \alpha_{i} K\left(\cdot, m_{i}\right)$. So $\mathcal{W}(\langle V\rangle)=\langle\{K(\cdot, m) \mid m \in \llbracket\}\rangle$. Moreover, it maps $\langle V\rangle$ isometrically onto $\langle\{K(\cdot, m) \mid m \in \llbracket\})$, because

$$
\begin{aligned}
& \left(\mathscr{W}\left(\sum_{i} \alpha_{i} \phi_{m_{i}}\right), W\left(\sum_{j} \beta_{j} \phi_{m_{j}^{\prime}}\right)\right)_{\mathbb{C}_{K}^{0}} \\
= & \left(\sum_{i} \alpha_{i} K\left(\cdot, m_{i}\right), \sum_{j} \beta_{j} K\left(\cdot, m_{j}^{\prime}\right)\right)_{\mathbb{C}_{K}^{0}} \\
= & \sum_{i, j} \bar{\alpha}_{i} \beta_{j} K\left(m_{i}, m_{j}^{\prime}\right)=\sum_{i, j} \bar{\alpha}_{i} \beta_{j}\left(\phi_{m_{i}}, \phi_{m_{j}^{\prime}}\right)_{H} .
\end{aligned}
$$


Since $\langle V\rangle$ is dense in $\overline{\langle V\rangle}$ and $\mathcal{W}$ is bounded on $H=$ $\overline{\langle V\rangle}$ it follows that $\mathcal{W}$ is an isometry. Furthermore, $\mathscr{W}[\langle V\rangle]$ is dense in $\mathbb{C}_{K}^{\natural}$. So $\mathcal{W}$ is also surjective and therefore unitary.

\section{APPENDIX C}

\section{NUMERICAL SCHEME FOR COMPUTING RESOLVENT OF EVOLUTIONS ON ORIENTATION SCORES}

We will first explain the numerical scheme for computing $W=-(A-\alpha I)^{-1} U_{f}$, with $A$ given by (8.81). This scheme (which we used in our experiments) is a generalization of the scheme proposed by Jonas August [5] for the directional process. This scheme is certainly preferable over the more usual finite difference approach as, we showed in [12 Appendix G1, pp. 5455]. For the sake of clarity in this Appendix, we use the symbols $\mathbf{x}=(x, y)$ rather than $\mathbf{b}=\left(b_{1}, b_{2}\right)$ for the spatial variables in $G$ in this Appendix section. Moreover, for the sake of simplicity, in this section we will write $U(\mathbf{b}, \theta)$ rather than $U\left(\mathbf{b}, e^{i \theta}\right)$. Consider the (discrete Fourier transform) basis functions

$$
\eta_{j k l}(x, y, \theta)=e^{2 \pi i\left(\frac{j x}{N_{1}}+\frac{k y}{N_{2}}+\frac{l \theta}{N_{3}}\right)},
$$

$j \in\left\{0, \ldots, N_{1}\right\}, k \in\left\{0, \ldots, N_{2}\right\}, l \in\left\{0, \ldots, N_{3}\right\}$. Since the spatial variables in our orientation bundles have the same dimensions as the spatial variables in the given $X \times Y$ image, we have $N_{1}=X$ and $N_{2}=Y$. Define the complex-valued constants $\hat{W}_{j, k, l}$ by

$$
\begin{gathered}
\hat{W}_{j, k, l}=\left(\eta_{j k l}, U_{f}\right)_{\mathbb{L}_{2}\left([0, X] \times[0, Y] \times S_{1}\right)} \\
=\iint_{0}^{X X 2 \pi} \int_{0} e^{-2 \pi i\left(\frac{j x}{X}+\frac{k y}{Y}+\frac{l \theta}{2 \pi}\right)} U_{f}(x, y, \theta) d \theta d x d y .
\end{gathered}
$$

Notice that by integration by parts

$$
\begin{gathered}
\left(\eta_{j k l}, \cos \theta \frac{\partial W}{\partial x}\right)=\int_{X \times Y \times S_{1}} \cos \theta W(x, y, \theta) \\
\times\left(\frac{2 \pi i j}{X}\right) e^{-2 \pi i\left(\frac{j x}{X}+\frac{k y}{Y}+\frac{l \theta}{2 \pi}\right)} d x d y d \theta
\end{gathered}
$$

now use the Euler formula $\cos \theta=\frac{e^{i \theta}+e^{-i \theta}}{2}$, and we see that

PATTERN RECOGNITION AND IMAGE ANALYSIS

Vol. $17 \quad$ No. $1 \quad 2007$

PATTERN RECOGNITION AND IMAGE ANALY

$$
\begin{aligned}
& \left(\eta_{j k l}, \cos \theta \frac{\partial W}{\partial x}\right)=-\frac{\pi i j}{X}\left(\hat{W}_{j k(l-1)}+\hat{W}_{j k(l+1)}\right) \\
& \left(\eta_{j k l}, \sin \theta \frac{\partial W}{\partial y}\right)=-\frac{\pi k}{Y}\left(\hat{W}_{j k(l-1)}+\hat{W}_{j k(l+1)}\right) .
\end{aligned}
$$

It now directly follows by (C.90) and (C.91) that

$$
\begin{gathered}
\left(\eta_{j k l}, \partial_{\xi} W\right)=\beta_{j, k} \hat{W}_{j k(l-1)}-\overline{\beta_{j, k}} \hat{W}_{j k(l+1)} \\
=\left[\beta_{j, k} S_{001}-\overline{\beta_{j, k}} S_{00-1}\right] \hat{W}_{j k l},
\end{gathered}
$$

with $\beta_{j, k}=\pi\left(\frac{i j}{X}+\frac{k}{Y}\right)$, where the shift operator $S_{a b c}, a$, $b, c \in \mathbb{Z}$ is given by $S_{a b c} \hat{W}_{j k l}=\hat{W}_{j-a, k-b, l-c}$. In an analogous manner, one can verify that

$$
\begin{gathered}
\left(\eta_{j k l}, \partial_{\eta} W\right)=\gamma_{j, k} \hat{W}_{j k(l-1)}-\overline{\gamma_{j, k}} \hat{W}_{j k(l+1)} \\
=\left[\gamma_{j, k} S_{001}-\overline{\gamma_{j, k}} S_{00-1}\right] \hat{W}_{j k l},
\end{gathered}
$$

with $\gamma_{j, k}=\pi\left(\frac{i k}{Y}-\frac{j}{X}\right)$. By (C.93) and (C.92), it now follows that

$$
\begin{aligned}
& \left(\eta_{j k l}, \partial_{\xi} \partial_{\xi} W\right)=\left[\beta_{j, k} S_{001}-\overline{\beta_{j, k}} S_{00-1}\right]^{2} \hat{W}_{j k l} \\
& =\left[\left(\beta_{j, k}\right)^{2} S_{002}+\left(\overline{\beta_{j, k}}\right)^{2} S_{00-2}-2\left|\beta_{j, k}\right|^{2}\right] \hat{W}_{j k l} \\
& \left(\eta_{j k l}, \partial_{\eta} \partial_{\eta} W\right)=\left[\gamma_{j, k} S_{001}-\overline{\gamma_{j, k}} S_{00-1}\right]^{2} \hat{W}_{j k l} \\
& =\left[\left(\gamma_{j, k}\right)^{2} S_{002}+\left(\overline{\gamma_{j, k}}\right)^{2} S_{00-2}-2\left|\gamma_{j, k}\right|^{2}\right] \hat{W}_{j k l} .
\end{aligned}
$$

Moreover, it follows by integration by parts that

$$
\begin{gathered}
\left(\eta_{j k l}, \partial_{\theta} W\right)=\frac{-i l}{N_{3}} \hat{W}_{j, k, l} \\
\text { and }\left(\eta_{j k l}, \partial_{\theta} \partial_{\theta} W\right)=-\left(\frac{l}{N_{3}}\right)^{2} \hat{W}_{j, k, l} .
\end{gathered}
$$

Notice that the operator $A$ leaves the spaces $\left\{\eta_{j, k, l}\right\}_{l \in Z}$ for fixed $j, k$ invariant and thereby the corresponding matrix is a block matrix:

$$
\begin{aligned}
& A=\left(\begin{array}{ccc}
A_{1,1} & 0 & 0 \\
0 & \ddots & 0 \\
0 & 0 & A_{N_{1} N_{2}}
\end{array}\right) \rightarrow(\alpha I-A)^{-1} \\
& =\left(\begin{array}{ccc}
\left(\alpha I-A_{1,1}\right)^{-1} & 0 & 0 \\
0 & \ddots & 0 \\
0 & 0\left(\alpha I-A_{N_{1}, N_{2}}\right)^{-1}
\end{array}\right) .
\end{aligned}
$$

It is now straightforward to compute the matrix $\alpha I-$ $A_{j, k}$ with respect to basis $\left\{\eta_{j, k, l}\right\}_{l \in\left(1, \ldots, N_{1}\right)}$, which is a 
5-band sparse matrix. The overall time complexity of computing $W$ from $U_{f}$ is $O(n \log n)$, where $n=N_{1} N_{2} N_{3}$. In practice, we often first compute the impulse response/Green function by means of the above algorithm and then compute the $G$-convolution ${ }^{20}(8.73)$.

\section{ACKNOWLEDGMENTS}

Special thanks to Prof. J. de Graaf, TUE Department of Mathematics, for his inspiring ideas concerning reproducing kernel theory and special thanks to Dr. A. F. M. ter Elst, TUE Department of Mathematics, for his explanation on group theoretical aspects.

\section{REFERENCES}

1. M. Abramowitz and I. A. Stegtun, Handbook of Mathematical Functions with Formulas, Graphs, and Mathematical Tables (Dover Publications, Inc., New York, 1965).

2. S. T. Ali, J. P. Antoine, and J. P. Gazeau, Coherent States, Wavelets and Their Generalizations (Springer Verlag, New York, Berlin, Heidelberg, 1999).

3. J. P. Antoine, "Directional Wavelets Revisited: Cauchy Wavelets and Symmetry Detection in Patterns," Applied and Computational Harmonic Analysis 6, 314-345 (1999).

4. N. Aronszajn, "Theory of Reproducing Kernels," Trans. A. M. S. 68, 337-404 (1950).

5. J. August, The Curve Indicator Random Field (PhD Thesis, Yale University, 2001).

6. J. August and S. W. Zucker, "The Curve Indicator Random Field and Markov Processes," IEEE-PAMI, Pattern Recognition and Machine Intelligence 25 (4), (2003).

7. W. H. Bosking, Y. Zhang, B. Schofield, and D. Fitzpatrick, "Orientation Selectivity and the Arrangement of Horizontal Connections in Tree Shrew Striate Cortex," The Journal of Neuroscience 17 (6), 2112-2127 (1997).

8. I. Daubechies, Ten Lectures on Wavelets. Number 61 in CBMS-NSF Series in Applied Mathematics (SIAM, Philadelphia, 1992).

9. M. Duits, "A Functional Hilbert Space Approach to Frame Transforms and Wavelet Transforms," MD Thesis in Applied Analysis. Eindhoven University of Technology, The Netherlands, September 2004.

10. M. Duits and R. Duits, "A Functional Hilbert Space Approach to the Theory of Wavelets," Technical Report, TUB, Eindhoven, March 2004. Technical Report RANA-7-2004, http://www.win.tue.nl/analysis/preprints/2004.html (Department of Mathematics Eindhoven University of Technology).

11. R. Duits, Perceptual Organization in Image Analysis. $\mathrm{PhD}$ Thesis, Eindhoven University of Technology, Department of Biomedical Engineering, The Netherlands, 2005. A digital version is available on the Web: URL: http://www.bmi2.bmt.tue.nl/Image_Analysis/People/RDuits/THESISRDUITS.pdf

12. R. Duits, M. Duits, and M. van Almsick, "Invertible Orientation Scores as an Application of Generalized Wavelet Theory," Technical report, TUE, Eindhoven, March 2004. Technical Report 04-04, Biomedical Image and

\footnotetext{
20 The $G$-convolutions/correlations can be adapted for each position $g=\left(\mathbf{b}, e^{i \theta}\right)$ in the orientation score.
}

Analysis (Department of Biomedical Engineering, Eindhoven University of Technology).

13. R. Duits, L. M. J. Florack, J. de Graaf, and B. ter Haar Romeny, "On the Axioms of Scale Space Theory," Journal of Mathematical Imaging and Vision 20, 267-298 (2004).

14. N. Dungey, A. F. M. Ter Elst, and D. W. Robinson, Analysis on Lie Groups with Polynomial Growth (BirkhauserProgress in Mathematics, Boston, 2003), Vol. 214.

15. S. J. L. Eijndhoven and J. de Graaf, "Some Results on Hankel Invariant Distribution Spaces," in Proceedings of the Koninklijke Akademie van Wetenschappen, Series A 86 (1), 77-87 (1982).

16. J. Faraut and K. Harzallah, Deux Cours D'analyse Harmonique (Birkhaeuser, Tunis, 1984).

17. M. Fashandi, R. A. Kamyabi Gol, A. Niknam, and M. A. Pourabdollah, "Continuous Wavelet Transform on a Special Homogeneous Space," Journal of Mathematical Physics 44 (9), 4260-4266 (2003).

18. L. M. J. Florack, Image Structure (Kluwer Academic Publishers, Dordrecht, The Netherlands, 1997).

19. A. Grossmann, J. Morlet, and T. Paul, "Integral Transforms Associated to Square Integrable Representations," J. Math. Phys. 26, 2473-2479 (1985).

20. C. J. Isham and J. R. Klauder, "Coherent States for $n$ Dimensional Euclidean Groups e (n) and Their Application," Journal of Mathematical Physics 32 (3), 607-620 (1991).

21. S. N. Kalitzin, B. M. ter Haar Romeny, and M. A. Viergever, "Invertible Apertured Orientation Filters in Image Analysis," International Journal of Computer Vision 31 (2/3), 145-158 (1999).

22. T. S. Lee, "Image Representation Using $2 \mathrm{~d}$ Gabor Wavelets," IE BE-Transactions on Pattern Analysis and Machine Intelligence 18 (10), 959-971 (1996).

23. A. K. Louis, P. Maass, and A. Rieder, Wavelets, Theory, and Applications (Wiley, New York, 1997).

24. F. J. L. Martens, "Spaces of Analytic Functions on Inductive/Projective Limits of Hilbert Spaces," $\mathrm{PhD}$ Thesis (University of Technology Eindhoven, Department of Mathematics and Computing Science, Eindhoven, The Netherlands, 1988). This PHD thesis is available on the webpages of the Technische Universiteit Eindhoven. Webpage in 2004: http://alexandria.tue.nl/extra3/proefschrift/PRF6A/8810117.pdf.

25. D. Mumford, Elastica and Computer Vision. Algebraic Geometry and Its Applications (Springer-Verlag, 1994), pp. 491-506.

26. W. Rudin, Functional Analysis (McGraw-Hill, New York, 1973).

27. M. Sugiura, Unitary Representations and Harmonic Analysis (North-Holland Mathematical Library, 44, Amsterdam), 2nd ed. (Kodansha, Tokyo, 1990).

28. D. Y. Ts'O, R. D. Frostig, E. E. Lieke, and A. Grinvald, "Functional Organization of Primate Visual Cortex Revealed by High Resolution Optical Imaging," Science 249, 417-420 (1990).

29. S. Twareque Ali, "A General Theorem on Square-Integrability: Vector Coherent States," Journal of Mathematical Physics 39 (8), (1998).

30. L. R. Williams and J. W. Zweck, "A Rotation and Translation Invariant Saliency Network," Biological Cybernetics, 88, 2-10 (2003). 


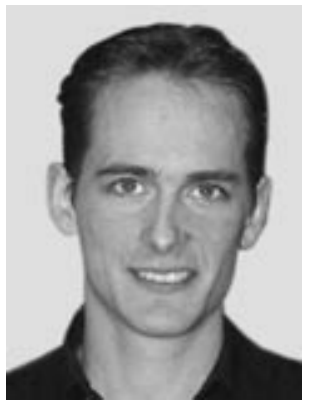

Remco Duits received his M.Sc. degree (cum laude) in Mathematics in 2001 from Eindhoven University of Technology, The Netherlands. He received his $\mathrm{PhD}$ degree (cum laude) at the Department of Biomedical Engineering at Eindhoven University of Technology on the subject of multiscale perceptual organization. His interests include functional analysis, group theory, partial differential equations, multiscale representations and their applications to biomedical imaging and vision, and perceptual grouping. His $\mathrm{PhD}$ thesis is titled Perceptual Organization in Image Analysis (A Mathematical Approach Based on Scale, Orientation and Curvature). Several of his submissions at conferences have been selected/best papers, in particular, at the PRIA 2004 conference on pattern recognition and image analysis in St. Petersburg, he received a best paper award (second prize) for his work on invertible orientation scores. Currently, he is working at Eindhoven University of Technology as an assistant professor at both the Department of Applied Mathematics and Computer Science and the Department of Biomedical Engineering.

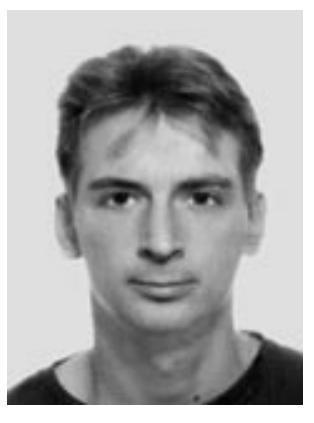

Maurice Duits received his MSc degree (cum laude) in Mathematics in 2004 from Eindhoven University of Technology, The Netherlands on the subject of reproducing kernels in frame and wavelet transforms. Now he is a PhD student at the Department of Mathematics at Katholieke Universiteit Leuven on the subject of random matrices. His interests include Riemann-Hilbert problems, random matrices, orthogonal polynomials and Toeplitz matrices.

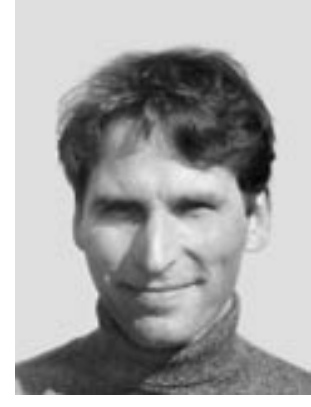

Markus van Almsick earned a master degree in physics at the Technical University of Munich in 1990. From 1988 until 1992, he worked for the University of Illinois at UrbanaChampaign as a research and teaching associate. He taught undergraduate chemistry as well as graduate courses in advanced quantum mechanics, for which he developed Mathematica course material. His research interest has been quantum logic and quantization procedures of space-time. Since 1990, he has been a freelance applications consultant for Wolfram Research, Inc., USA, and Wolfram Research Europe Ltd., United Kingdom, promoting Mathematica at universities and research institutions in the U.S., Europe, and Israel, as well as developing Mathematica packages and application material. In 1996, Mr. van Almsick joined the Max Planck Insitut fur Biophysik in Frankfurt am Main, Germany, where he addressed problems in nonequilibrium thermodynamics until the theoretical department closed in 1997. Then, until 2001 he worked in collaboration with QT Software $\mathrm{GmbH}$, Munich, as a full-time Mathematica consultant on a wide variety of assignments, e.g., designing the geometry of slides for playgrounds, modeling human interaction via graph theory ("social networks"), lossless image compression, vibration control in electric engines, and the isomer enumeration of libraries containing chemical diamutamers. Since 2001, he has been a part-time employee of the Technische Universiteit Eindhoven, where he develops MathVisionTools, a biomedical image analysis toolkit based on Mathematica.

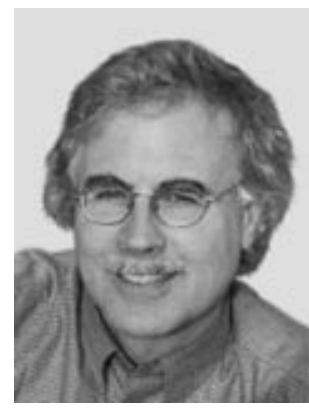

Bart M. ter Haar Romany is full professor in Biomedical Image Analysis at the Department of Biomedical Engineering at Eindhoven University of Technology. He has been in this position since 2001. He received a MSc in Applied Physics from Delft University of Technology in 1978, and a PhD on neuromuscular nonlinearities from Utrecht University in 1983. After being the principal physicist of the Utrecht University Hospital Radiology Department, in 1989 he joined the department of Medical Imaging at Utrecht University as an associate professor. His interests are mathematical aspects of visual perception, in particular linear and non-linear scale-space theory, computer vision applications, and all aspects of medical imaging. He is author of numerous papers and book chapters on these issues; he edited a book on non-linear diffusion theory and is author of an interactive tutorial book on scale-space theory in computer vision. He has initiated a number of international collaborations on these subjects. He is an active teacher in international courses, a senior member of IEEE, and IEEE Chapter Tutorial Speaker. He is chairman of the Dutch Biophysical Society. 